\title{
Invariance of immersed Floer cohomology under Maslov flows
}

\author{
JOSEPH PALMER AND CHRIS WOODWARD
}

\begin{abstract}
We show that immersed Lagrangian Floer cohomology in compact rational symplectic manifolds is invariant under Maslov flow; this includes coupled mean curvature/Kähler-Ricci flow in the sense of Smoczyk [52]. In particular, we show invariance when a pair of self-intersection points is born or dies at a self-tangency, using results of Ekholm-Etnyre-Sullivan [23]. Using this we prove a lower bound on the time for which the immersed Floer theory is invariant under the flow, if it exists. This proves part of a conjecture of Joyce [38].
\end{abstract}

\section{Contents}

1 Introduction 2

2 Maslov flows of Lagrangian immersions $\quad 8$

3 Holomorphic disks with self-transverse boundary condition 20

4 Transversality and compactness $\quad 30$

5 Holomorphic disks with self-tangent boundary condition 46

6 Morse model for immersed Floer cohomology 53

$7 \quad$ Invariance for transverse self-intersection 63

8 Curve shrinking and gluing at a tangency $\quad 70$

9 Invariance for birth-death singularities $\quad 85$ 


\section{Introduction}

Lagrangian Floer theory is a cohomology theory whose differential counts pseudoholomorphic disks in a symplectic manifold with Lagrangian boundary conditions. For many purposes one wants to consider Floer theory of not only embedded but also immersed Lagrangians as introduced by Akaho-Joyce [2]. In Calabi-Yau manifolds, one expects special Lagrangians to play a special role as in the Thomas-Yau conjecture [57], namely, they should split-generate the Fukaya category. In order to obtain special Lagrangians, one may hope to start with an arbitrary Lagrangian and minimize the volume by evolving under the mean curvature flow. Unfortunately, there is no reason to expect mean curvature flow of submanifolds of codimension greater than one to preserve embeddedness. In addition, although short-time solutions to the mean curvature flow exist [7], in general surgery is required to continue the flow beyond singular times, as in Colding-Minicozzi [19] and for the Lagrangian torus case Chen-Ma [17].

From the point of view of mirror symmetry, mean curvature flow for Lagrangian branes is expected to be mirror to a (deformed version of) Yang-Mills flow for vector bundles or coherent sheaves. Since Yang-Mills flow for vector bundles is achieved by a complex gauge transformation, one expects the isomorphism class of a Lagrangian brane to be invariant under the mean curvature flow. In this paper, we prove several results in this direction. We consider any flow whose deformation class at any time is equal to the Maslov class, and call such flows Maslov flows; a mean curvature flow, if it exists, is a special case. We prove invariance of Floer cohomology until the first singularity occurs or until the weakly bounding cochain hits a wall of zero valuation; in particular we show invariance of the Floer cohomology in the case that the (coupled, forward or reverse) mean curvature flow exists and passes through a self-tangency. We have in mind an application to the existence of Floer non-trivial Lagrangians (and generators for the Fukaya category) in Kähler surfaces that we will discuss elsewhere. In particular this proves "step zero" in the conjectures of Joyce [37], [38] for the existence of Lagrangian mean curvature flow with surgery for weakly unobstructed Lagrangians. To explain the terminology, recall from Akaho-Joyce [2] that associated to a self-transverse immersion $\phi: L \rightarrow X$ of a compact Lagrangian in a compact symplectic manifold $X$ is a Fukaya $A_{\infty}$ algebra $C F(\phi)$ additively generated over a Novikov ring by chains on the Lagrangian plus two copies of each self-intersection; for each $d \geq 0$ the composition maps

$$
\mu_{d}: C F(\phi)^{\otimes d} \rightarrow C F(\phi)
$$




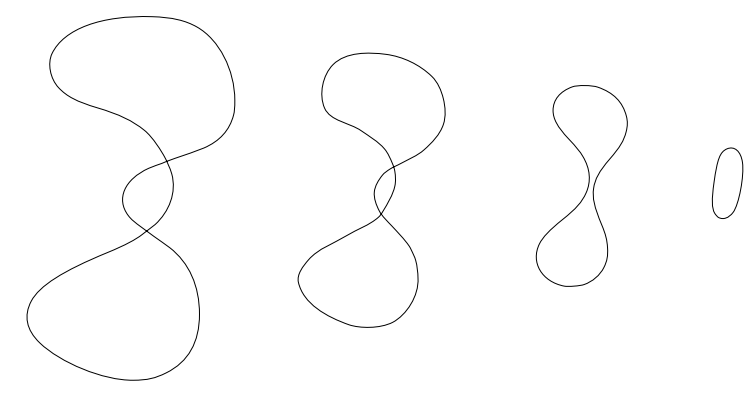

Figure 1: An immersed curve flowing under the mean curvature flow.

count holomorphic disks with boundary in the Lagrangian. Via the homotopy units construction in [31, (3.3.5.2)], [16, Section 2.2] one may furnish $C F(\phi)$ with a strict unit $1_{\phi} \in C F(\phi)$. A bounding cochain (resp. weakly bounding cochain) is a solution $b \in C F(\phi)$ to the Maurer-Cartan equation (resp. weak Maurer-Cartan equation)

$$
\mu_{0}(1)+\mu_{1}(b)+\mu_{2}(b, b)+\ldots=0\left(\text { resp. }=W(b) 1_{\phi}\right) .
$$

The space of solutions to the weak Maurer-Cartan equation is denoted $M C(\phi)$ and is equipped with a potential function

$$
M C(\phi) \rightarrow \Lambda, \quad b \mapsto W(b) .
$$

For any $b \in M C(\phi)$, the operator

$$
\mu_{1}^{b}: C F(\phi) \rightarrow C F(\phi), \quad c \mapsto \sum_{k_{-} \geq 0, k_{+} \geq 0} \mu_{k_{-}+k_{+}+1}(\underbrace{b, \ldots, b}_{k_{-}}, c, \underbrace{b, \ldots, b}_{k_{+}})
$$

squares to zero. Non-vanishing of the Floer cohomology for some weakly bounding cochain obstructs the Hamiltonian displaceability of the Lagrangian. That is, if there exists a Hamiltonian diffeomorphism $\psi: X \rightarrow X$ such that $\psi(\phi(L)) \cap \phi(L)$ is empty then the Floer cohomology $H F(\phi, b)$ vanishes for any $b \in M C(\phi)$, or $M C(\phi)$ is empty.

We study the behavior of immersed Floer cohomology under flows that in a cohomological sense are equivalent to forward or reverse mean curvature flow coupled with Kähler-Ricci flow on the symplectic manifold. Consider a one-parameter family $\phi_{t}: L \rightarrow X$ of Lagrangian immersions for a family of symplectic forms $\omega_{t}$ such that the deformation class $\operatorname{Def}\left(\phi_{t}\right)=\left[\dot{\phi}_{t},-\dot{\omega}_{t}\right]$ as defined in Equation (7) satisfies

$$
\operatorname{Def}\left(\phi_{t}\right)= \pm c_{1}\left(\phi_{t}\right) \in H^{2}\left(\phi_{t}\right)
$$

where $c_{1}\left(\phi_{t}\right)$ is the Maslov (relative Chern) class $c_{1}\left(\phi_{t}\right)$ in the relative de Rham cohomology $H^{2}\left(\phi_{t}\right)$ defined in (6) below. We call such a flow a Maslov flow (without 
surgery) as in Lotay-Pacini [42] (up to a sign). In particular, this implies that

$$
\left[\dot{\omega}_{t}\right]= \pm c_{1}(X)
$$

with $c_{1}(X) \in H^{2}(X)$ the first Chern class, as in a Kähler-Ricci flow. It is an observation of Smoczyk [52] that the combined mean curvature and Kähler-Ricci flow preserves the Lagrangian condition. A result of Lotay-Pacini [42, Section 7] (using a technique of Hamilton) says that such coupled mean-curvature flows $\phi_{t}: L \rightarrow X, \omega_{t}$ exist for short time. All symplectic manifolds $X$ will be assumed to be compact and Lagrangians $L$ will be assumed to be compact, oriented, and equipped with relative spin structures and local systems. In order to apply Cieliebak-Mohnke perturbations [15] we furthermore assume that $X$ is simply-connected and the relative symplectic class $[0, \omega] \in H^{2}(\phi, \mathbb{Q})$ is rational (which will hold for rational times in the flow).

At times when the number of self-intersection points changes, we find a correction to the weakly bounding cochain so that the Floer cohomology and potential is preserved. Suppose that under a flow $\phi_{t}$ two new self-intersection points $x_{1, t}, x_{2, t} \in \phi\left(L_{t}\right)$ are born at $t=0$, leading to four ordered self-intersection points $v_{t, \pm}, \bar{v}_{t, \pm} \in L \times_{\phi_{t}} L{ }^{1}$ In our conventions, $\bar{v}_{t, \pm}$ is connected by a Floer trajectory to $v_{t, \mp}$ as in Figure 2 by Theorem 8.1 .

Theorem 1.1 (Invariance for self-tangencies) Let $\left(\phi_{t}\right)_{t \in[-\epsilon, \epsilon]}$ be a (forward or reverse) Maslov flow (as in Definition 2.2) of Lagrangian immersions developing a tangency $D \phi_{t}\left(T_{l_{-}} L\right) \cap D \phi_{t}\left(T_{l_{+}} L\right) \neq\{0\}$ at $t=0$ with two additional self-intersections $x_{1, t}, x_{2, t}$ for $t>0$. For any family

$$
b_{t,-} \in M C\left(\phi_{t}\right), \quad t \in[-\epsilon, 0]
$$

of Maurer-Cartan solutions for $C F\left(\phi_{t}\right), t<0$ with $\operatorname{val}_{q}\left(b_{0}\right)>0$, there exists a family

$$
b_{t,+} \in M C\left(\phi_{t}\right), \quad t \in[0, \epsilon]
$$

of Maurer-Cartan solutions for $C F\left(\phi_{t}\right)$ for $t \in(0, \epsilon)$ such that

$$
\lim _{t \rightarrow 0^{+}} W\left(\phi_{t}, b_{t,+}\right)=\lim _{t \rightarrow 0^{-}} W\left(\phi_{t}, b_{t,-}\right)
$$

and there is an isomorphism of Floer cohomologies for $-\epsilon<t_{-}<0<t_{+}<\epsilon$

$$
H F\left(\phi_{t_{-}}, b_{t_{-},-}\right) \rightarrow H F\left(\phi_{t_{+}}, b_{t_{+},+}\right) .
$$

\footnotetext{
${ }^{1}$ We use a bar over an ordered intersection point if the orientation induced by the splitting into tangent spaces of the branches of the Lagrangian disagrees with the symplectic orientation, and no bar otherwise.
} 


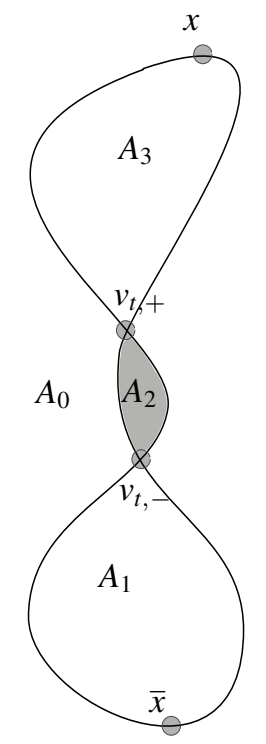

Figure 2: Small strips created by intersection points

A very similar argument for the invariance of Legendrian contact homology appears in Ekholm-Etnyre-Sullivan [23], and our proof uses the same basic idea although its realization in the language of Lagrangian Floer theory is somewhat different. In particular Ekholm-Etnyre-Sullivan [23, Lemma 4.6] describes the behavior of holomorphic disks with boundary in a Lagrangian immersion under the development of a self-tangency. As in that paper, we work in a local model in which the self-tangency is "of standard form", justified in Lemma 2.8. We consider families

$$
b_{t, \pm} \in C F\left(\phi_{t}\right), t \in[ \pm \epsilon, 0]
$$

which on either side of the tangency are connected by a correction formula

$$
b_{t,+}=E_{t_{-}}^{t_{+}} b_{t,-}+\sum_{ \pm} q^{-A(t)} \# \mathcal{M}\left(\phi_{t_{+}}, b_{t,-} ; v_{t, \pm}\right) \bar{v}_{t, \mp}
$$

where $E_{t_{-}}^{t_{+}}$is an operator acting by multiplication by some power of $q$ in each graded piece (see Definition 54) $A(t)$ is the area of the small strip connecting $v_{t, \mp}$ with $v_{t, \pm}$ and, as usual, the count is a signed count weighted by the areas of the disks. In the case that the immersion stays self-transverse, we identify the Maurer-Cartan solution spaces and the Floer cohomology for small times.

Theorem 1.2 (Invariance for self-transverse families) Let $\left(X, \omega_{0}\right)$ be a compact, ra- 
tional, simply-connected symplectic manifold as above and

$$
\omega_{t} \in \Omega^{2}(X), \quad \phi_{t}: L \rightarrow X, \quad t \in[0, T]
$$

a Maslov flow of symplectic forms and self-transverse Lagrangian immersions. Given $b_{0} \in M C\left(\phi_{0}\right)$ with $\operatorname{val}_{q}\left(b_{0}\right)>(\operatorname{dim}(L)-1) T$ there exists $b_{T} \in M C\left(\phi_{T}\right)$ such that

$$
W\left(\phi_{T}, b_{T}\right)=q^{2 T} W\left(\phi_{0}, b_{0}\right), \quad H F\left(\phi_{T}, b_{T}\right) \cong H F\left(\phi_{0}, b_{0}\right)
$$

where $\cong$ denotes group isomorphism. Similarly, given $b_{T} \in M C\left(\phi_{T}\right)$ with $\operatorname{val}_{q}\left(b_{T}\right)>$ $2 T$ there exists $b_{0} \in M C\left(\phi_{0}\right)$ such that (3) holds.

In the monotone case, invariance of Floer cohomology in this setting is a result of Alston-Bao [3]. The reason for the asymmetry in the two cases in the Theorem (that is, the difference between the bounds $(\operatorname{dim}(L)-1) T$ versus $2 T)$ lies in the fact that the necessary modification changes the weakly bounding cochain in degree $d$ by a multiple of $\pm(d-1) T$; that is, there is a symmetry of the factors around degree 1 weakly bounding cochains which roughly correspond to local systems and so require no modification under the flow.

Combining Theorems 1.1 and 1.2 above, consider a situation in which a Maslov flow of Lagrangian immersions $\phi_{t}: L \rightarrow X$ exists on some time interval $[0, T]$. After perturbation (see Lemma 2.8 below) we may assume that $\phi_{t}$ is self-transverse except for a finite collection of times $t_{1}, \ldots, t_{k} \in[0, T]$ at which self-tangencies occur. Let

$$
A_{i}=\operatorname{val}_{q}\left(b_{t_{i},+}-b_{t_{i},-}\right)
$$

be the $q$-valuation of the correction term $b_{t_{i},+}-b_{t_{i},-}$ in (2) at the self-tangency $t_{i}$, for $i=1, \ldots, k$ and define $A_{0}=\operatorname{val}_{q}\left(b_{0}\right)$.

Theorem 1.3 Let $\left(X, \omega_{0}\right)$ be a compact, rational, simply-connected symplectic manifold as above and

$$
\omega_{t} \in \Omega^{2}(X), \quad \phi_{t}: L \rightarrow X, \quad t \in[0, T]
$$

a Maslov flow of symplectic forms and Lagrangian immersions that are self-transverse for $t \in\{0, T\}$. Given $\left[b_{0}\right] \in \overline{M C}\left(\phi_{0}\right)$ with

$$
\min _{i=0}^{k}\left(A_{i}-(\operatorname{dim}(L)-1)\left(T-t_{i}\right)\right)>0
$$

resp. $\left[b_{T}\right] \in \overline{M C}\left(\phi_{T}\right)$ with

$$
\min \left(\operatorname{val}_{q}\left(b_{T}\right)-2 T, \min _{i=1}^{k} A_{i}-2\left(T-t_{i}\right)\right)>0
$$


there exists $\left[b_{T}\right] \in \overline{M C}\left(\phi_{T}\right)$ resp. $\left[b_{0}\right] \in \overline{M C}\left(\phi_{0}\right)$ such that

$$
W\left(\phi_{T}, b_{T}\right)=q^{2 T} W\left(\phi_{0}, b_{0}\right) \quad H F\left(\phi_{T}, b_{T}\right) \cong H F\left(\phi_{0}, b_{0}\right)
$$

where $\cong$ denotes group isomorphism.

Example 1.4 The following example shows that Floer theory can be initially unobstructed but become obstructed under mean curvature flow. Let the symplectic manifold $X$ be the two-sphere $S^{2}$, thought of as the one-point compactification of a plane $\mathbb{R}^{2}$, equipped with a metric that is flat on a large open subset $U \subset \mathbb{R}^{2}$ containing the image $\phi(L)$ of the immersion $\phi: L \cong S^{1} \rightarrow X$. A "movie" showing a family of circles $\phi_{t}: L \rightarrow X$ under the mean curvature flow $\dot{\phi}_{t}=-H_{\phi_{t}}$ is shown in Figure 3. ${ }^{2}$ Indeed, initially the area of the middle bigon is smaller than the areas of the teardrops; we will see when we analyze this example in more detail later (Example 6.5) that there exists a weakly bounding cochain $b_{t} \in M C\left(\phi_{t}\right)$. On the other hand, once the area of the bigon becomes larger than the combined area of the teardrops then no choice of weakly bounding cochain $b_{t}$ can cancel the teardrop contributions to $\mu_{0}(1) \in C F\left(\phi_{t}\right)$. By rescaling this example one sees that similarly, Floer cohomology $\operatorname{HF}\left(\phi_{t}, b_{t}\right)$ can not be invariant under arbitrary exact isotopy, that is, deformations $\phi_{t}$ generated by exact one-forms $\alpha_{t} \in \Omega^{1}(L)$. In higher dimension, we show in [48] that one can continue the flow by performing a surgery at one of the intersection points, at least in high dimensions. This ends the example.

Mean curvature flow for curves is also known as the curve-shortening flow. In this case, for embedded curves in the plane $\phi_{0}: L \rightarrow X=\mathbb{R}^{2}$ Grayson [34] proved that the curve $\phi_{t}(L)$ eventually becomes convex and then collapses to a point in finite time. For immersed curves $\phi_{0}: L \rightarrow X$ Angenent-Velázquez [4] studied the development of cusps. Angenent [5] studied further the curve-shortening flow on arbitrary surfaces $X$. Even for curves in the Euclidean plane, Theorem 1.3 includes a statement that is not obvious to the authors:

Corollary 1.5 Suppose $\phi: S^{1} \rightarrow \mathbb{R}^{2}$ is an immersion such that the Maurer-Cartan moduli space $M C(\phi)$ is empty. Then the curve-shortening flow of $\phi$ encounters a singularity, that is, does not flow to a round point in the sense of Grayson [34].

Non-emptiness of the Maurer-Cartan space does not imply convergence to a point, by Example (1.4). Joyce's conjecture [38] also makes sense for the curve-shortening flow for immersed curves in the two-sphere $S^{2}$, and is open even in that case. Work of

\footnotetext{
${ }^{2}$ Produced using the curve-shortening software by A. Carapetis [10] and Inkscape.
} 


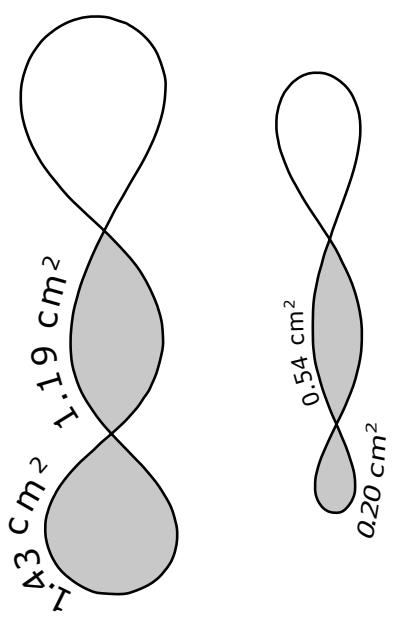

Figure 3: A flow in which the Floer cohomology becomes obstructed, with areas indicated

Angenent and others [5] describes the formation of singularities but note that Joyce's suggested surgery should be performed before the singularities arise, see Remark 9.8 and the sequel to this paper [48].

The intended application of these results is to the Lagrangian minimal model program as explained in Charest-Woodward [16]. The conjecture in [16] describes an orthogonal decomposition of the split-closed derived Fukaya category $D^{\pi} \mathcal{F}(X)$ of a Kähler manifold $X$ corresponding to singularities in the Kähler-Ricci flow-with-surgery; that is, a family of Kähler manifolds $\left(X_{t}, \omega_{t}\right)$ satisfying $\dot{\omega}_{t}=-R_{\omega_{t}}$ except at finitely many times where flips, divisorial contractions, or a fibration occur. Floer non-trivial Lagrangians associated to the singularities in the flow were described in [16]; the results of this paper imply the invariance of the Floer cohomology as these Lagrangians are "flowed backwards" under the Kähler-Ricci flow. We address in [48] the invariance of Floer cohomology under simplest kind of surgery. We close the paper with Remark 9.8, which lists some related open questions.

\section{Maslov flows of Lagrangian immersions}

Recall basic definitions regarding Lagrangian immersions following, for example, Weinstein [61]. Let $(X, \omega)$ be a symplectic manifold. A Lagrangian immersion is a 
smooth map $\phi: L \rightarrow X$ from a smooth manifold $L$ to $X$ satisfying

$$
\phi: L \rightarrow X, \quad \operatorname{dim}(L)=\operatorname{dim}(X) / 2, \quad \phi^{*} \omega=0, \quad \operatorname{ker}\left(D_{x} \phi\right)=\{0\}, \forall x \in L .
$$

The set of ordered self-intersection points is

$$
\mathcal{I}^{\text {si }}(\phi)=L \times_{\phi} L-\Delta_{L}
$$

where $\Delta_{L}: L \rightarrow L \times L$ is the diagonal embedding. A Lagrangian immersion $\phi$ : $L \rightarrow X$ from a compact manifold $L$ is a Lagrangian embedding if $\phi$ is injective, or equivalently if $\mathcal{I}^{\text {si }}(\phi)$ is empty. Lagrangian immersions are closed under the disjoint union construction: If $\phi_{0}: L_{0} \rightarrow X$ and $\phi_{1}: L_{1} \rightarrow X$ are Lagrangian immersions then so is the disjoint union

$$
\phi_{0} \sqcup \phi_{1}: L_{0} \sqcup L_{1} \rightarrow X, \quad x \mapsto\left\{\begin{array}{ll}
\phi_{0}(x) & x \in L_{0} \\
\phi_{1}(x) & x \in L_{1}
\end{array} .\right.
$$

Locally any Lagrangian immersion is the disjoint union of Lagrangian embeddings, so that the self-intersections of the union $\phi_{0} \sqcup \phi_{1}$ include the intersections between different components $\phi_{0}\left(L_{0}\right), \phi_{1}\left(L_{1}\right)$. Similarly, the product of Lagrangian immersions $\phi_{0}: L_{0} \rightarrow X_{0}$ and $\phi_{1}: L_{1} \rightarrow X$ is a Lagrangian immersion to $X_{0} \times X_{1}$.

A natural equivalence on the set of Lagrangian immersions is given by Lagrangian isotopy. Two Lagrangian immersions $\phi_{0}, \phi_{1}: L \rightarrow X$ are isotopic if there exists a family

$$
\phi_{t}: L \rightarrow X, t \in[0,1]
$$

of Lagrangian immersions connecting them, smooth in the parameter $t$. The derivative of an isotopy of Lagrangian immersions $\phi_{t}: L \rightarrow X$ at any time $t \in[0,1]$ may be identified with a closed one-form on the domain as follows: The quotient $\phi_{t}^{*} T X / T L$ is the normal bundle of the immersion. Consider the normal vector field

$$
v_{t}: L \rightarrow \phi_{t}^{*} T X / T L, \quad v_{t}(l)=\frac{d}{d t} \phi_{t}(l) \bmod D \phi_{t}\left(T_{l} L\right) .
$$

The pairing $\iota\left(v_{t}\right) \phi_{t}^{*} \omega$ is well-defined since $\phi_{t}$ is Lagrangian. The derivative of the pull-back of the symplectic form satisfies

$$
\frac{d}{d t}\left(\phi_{t}^{*} \omega\right)=\mathrm{d} \dot{\phi}_{t}+\iota\left(v_{t}\right) \phi_{t}^{*} \mathrm{~d} \omega
$$

where

$$
\dot{\phi}_{t}:=\omega\left(D \phi_{t}(\cdot), v_{t}\right) \in \Omega^{1}(L) .
$$

This follows locally from the Cartan homotopy formula and the fact that in the embedded case $\omega\left(D \phi_{t}(\cdot), v_{t}\right)=\phi_{t}^{*} \iota\left(\tilde{v}_{t}\right) \omega$ where $\tilde{v}_{t}$ is an extension of $v_{t}$ to a neighborhood of 
$\phi_{t}(L)$. Since $\phi_{t}^{*} \omega$ and $\mathrm{d} \omega$ are both zero, $\dot{\phi}_{t}$ is closed for all $t \in[0,1]$. Its cohomology class is the deformation class of the isotopy

$$
\operatorname{Def}\left(\phi_{t}\right)=\left[\dot{\phi}_{t}\right] \in H^{1}(L)
$$

If $\psi_{t}: X \rightarrow X, t \in[0,1]$ is a smooth family of symplectomorphisms then $\psi_{t} \circ \phi_{0}$ : $L \rightarrow X$ provides an isotopy from $\phi_{0}$ to $\psi_{1} \circ \phi_{0}$. If $\psi_{t}: X \rightarrow X, t \in[0, T]$ is a family of Hamiltonian symplectomorphisms then we say that the immersions $\phi_{t}=\psi_{t} \circ \phi_{0}$ are Hamiltonian isotopic and we write $\phi_{0} \sim \phi_{1}$. In this case, the deformation class is trivial. The Gromov-Lees h-principle [41], [36] classifies isotopy classes of Lagrangian immersions by the homotopy classes of their tangent maps $D \phi: T L \rightarrow T X$ :

$$
\phi_{0} \sim \phi_{1} \Longleftrightarrow D \phi_{0} \simeq D \phi_{1} .
$$

So in particular, immersions of a circle $S^{1}$ into the plane $\mathbb{R}^{2}$ are classified up to isotopy by the winding numbers of their Gauss map, while there are two isotopy classes of immersions of a circle $S^{1}$ into the two-sphere $S^{2}$.

To study further Lagrangian isotopies of immersions we recall the definition of the relative de Rham complex as in Bott-Tu [9, Section 6]. For $k \geq 0$ denote the space of relative forms

$$
\Omega^{k}(\phi):=\Omega^{k-1}(L) \oplus \Omega^{k}(X) .
$$

Equip $\Omega^{k}(\phi)$ with the relative de Rham differential the operator of order 1 given by

$$
\mathrm{d}: \Omega(\phi) \rightarrow \Omega(\phi), \quad\left(\beta_{L}, \beta_{X}\right) \mapsto\left(-\mathrm{d} \beta_{L}+\phi^{*} \beta_{X}, \mathrm{~d} \beta_{X}\right) .
$$

The relative de Rham cohomology is

$$
H(\phi)=\bigoplus_{k=0}^{m(X, L)} H^{k}(\phi), \quad H^{k}(\phi)=\frac{\operatorname{ker}(\mathrm{d}) \cap \Omega^{k}(\phi)}{\operatorname{im}(\mathrm{d}) \cap \Omega^{k}(\phi)}
$$

$m(X, L)=\max (\operatorname{dim}(X), \operatorname{dim}(L))$. The relative cohomology fits into a long exact sequence

$$
\ldots \rightarrow H^{k}(\phi) \rightarrow H^{k}(X) \rightarrow H^{k}(L) \rightarrow H^{k+1}(\phi) \rightarrow \ldots
$$

Relative cocycles integrate naturally over any relative cycle. Such a cycle is a pair of maps from a manifold and its boundary:

$$
u: S \rightarrow X, \quad \partial u: \partial S \rightarrow L, \quad \phi \circ \partial u(z)=u(z), \forall z \in \partial S .
$$

Integration of a cocycle $\left(\beta_{L}, \beta_{X}\right) \in \Omega^{k}(\phi)$ where $k=\operatorname{dim}(S)$ is given by

$$
\int_{u}\left(\beta_{L}, \beta_{X}\right)=\int_{S} u^{*}\left(\beta_{X}\right)-\int_{\partial S}(\partial u)^{*}\left(\beta_{L}\right) .
$$


By Stokes' theorem, the integral (5) depends only on the cohomology class $\left[\beta_{L}, \beta_{X}\right] \in$ $H^{k}(\phi)$.

In particular the relative symplectic form defines a map on relative second homology of the immersion. Let $\phi: L \rightarrow X$ be a Lagrangian immersion. The pair $(0, \omega) \in \Omega^{2}(\phi)$ is a relative de Rham cocycle since

$$
\phi^{*} \omega=0, \quad \mathrm{~d} \omega=0 .
$$

Denote the unit disk in the complex plane

$$
S=\{z \in \mathbb{C}|| z \mid \leq 1\} .
$$

A disk with boundary $\phi$ is a pair consisting of a map $u: S \rightarrow X$ and a lift of the restriction to the boundary $\left.u\right|_{\partial S}$ to a map to $L$ :

$$
u: S \rightarrow X, \quad \partial u: \partial S \rightarrow L, \quad \phi \circ \partial u=\left.u\right|_{\partial S}
$$

The homomorphisms associated to the symplectic and Maslov classes are denoted as follows. Given a disk $u:(S, \partial S) \rightarrow(X, L)$ the symplectic area $A(u)$ is the pairing of $[\omega]$ with the class of $[S, \partial S]$ and induces a map

$$
A: H_{2}(\phi) \rightarrow \mathbb{R}, \quad[u] \mapsto \int_{D} u^{*} \omega .
$$

The Maslov (or relative Chern) index measures the winding of the Lagrangian boundary condition. To explain the definition (6) let $\operatorname{dim}(X)=2 n$ for some positive integer $n$. Let $U(n)$ resp. $S O(n)$ denote the space of unitary resp. special orthogonal $n \times n$ matrices and let

$$
\operatorname{Lag}\left(\mathbb{C}^{n}\right) \cong U(n) / S O(n)
$$

denote the Grassmannian of oriented Lagrangian subspaces of $\mathbb{C}^{n}$. Since the disk $S$ is contractible, there exists a trivialization of symplectic vector bundles $u^{*} T X \cong S \times \mathbb{C}^{n}$, unique up to isomorphism. Let

$$
(\partial u)^{*} T L: \partial S \rightarrow \operatorname{Lag}\left(\mathbb{C}^{n}\right)
$$

denote the family of Lagrangian, or equivalently, totally real subspaces on the boundary of the disk $\partial S$. The determinant det $: U(n) \rightarrow U(1)$ factors through $U(n) / S O(n)$ and induces a loop whose winding number is by definition half the Maslov index:

$$
I: H_{2}(\phi) \rightarrow \mathbb{Z}, \quad[u] \mapsto 2\left[\operatorname{det}\left((\partial u)^{*} T L\right)\right] \in \pi_{1}\left(S^{1}\right) \cong \mathbb{Z} .
$$

In [43, Appendix C] Robbin shows that the Maslov index for bundles is the unique invariant that is additive under direct sum, additive under sewing boundaries and suitably normalized for line bundles over the disk. It follows that the Maslov index 
map $[u] \mapsto I([u])$ is the relative Chern class of $T X$ (as pointed out by T. Perutz) and defines a cohomology class denoted $c_{1}(\phi) \in H^{2}(\phi)$. Alternatively, the explicit differential form representing the Maslov class [47] implies that the Maslov class is a relative cohomology class.

In the case of isotopies in which the symplectic form varies, the deformation of the Lagrangian and symplectic form combine to a relative cocycle. Let

$$
\omega_{t} \in \Omega^{2}(X), t \in[0,1], \quad \text { resp. } \phi_{t}: L \rightarrow X
$$

be a family of symplectic forms resp. a family of Lagrangian immersions for $\omega_{t}$. The derivative of the pullback symplectic form satisfies

$$
0=\frac{d}{d t}\left(\phi_{t}^{*} \omega_{t}\right)=\mathrm{d} \dot{\phi}_{t}+\phi_{t}^{*} \dot{\omega}_{t}
$$

by an argument similar to that of (4). The deformation class of the isotopy is an element in the relative de Rham cohomology

$$
\operatorname{Def}\left(\phi_{t}\right)=\left[\dot{\phi}_{t},-\dot{\omega}_{t}\right] \in H^{2}\left(\phi_{t}\right) .
$$

If the class (7) vanishes, then the isotopy is an exact isotopy. The notions of immersed Lagrangian isotopy resp. exact Lagrangian isotopy $\phi_{0} \simeq \phi_{T}$ are easily shown to be equivalence relations; concatenation of isotopies can be taken to be smooth by deforming so that the isotopies are constant near the starting and ending times $t=0, T$.

Deformations of Lagrangian isotopies by the Maslov class arise from a choice of connection one-form on the anti-canonical bundle. Recall that the tangent bundle $T X$ to the symplectic manifold $X$ has a complex structure well-defined up to isotopy. The top exterior power of the dual $T^{\vee} X$ of $T X$

$$
K^{-1}=\Lambda_{\mathbb{C}}^{\text {top }}\left(T^{\vee} X\right)
$$

is the anticanonical bundle. By definition the first Chern class of $K^{-1}$ is the first Chern class of $X$. Assume that $L$ is oriented by a section of the orientation bundle

$$
o_{L}: L \rightarrow \Lambda_{\mathbb{R}}^{\mathrm{top}}\left(T^{\vee} L\right) \text {. }
$$

Since $T L$ is a totally real sub-bundle of $\phi^{*} T X$ one has an isomorphism

$$
\phi^{*} T X \cong T L \otimes_{\mathbb{R}} \mid C .
$$

This isomorphism of bundles induces an isomorphism of top exterior powers

$$
\Lambda_{\mathbb{R}}^{\text {top }}\left(T^{\vee} L\right) \otimes_{\mathbb{R}} \mathbb{C} \rightarrow \Lambda_{\mathbb{C}}^{\text {top }}\left(\phi^{*} T^{\vee} X\right)
$$

In this way the orientation $o_{L}$ on $L$ induces a trivialization

$$
\phi^{*} K^{-1} \rightarrow L \times \mathbb{C}
$$


of the pull-back of the anticanonical bundle $K^{-1}$. Let $\alpha_{X}$ be a connection one-form on $K^{-1}$. By definition,

$$
\alpha_{X} \in \Omega\left(K_{1}^{-1}\right)^{S^{1}}, \quad \alpha_{X}(\partial / \partial \theta)=1
$$

is an $S^{1}$-invariant one-form on the unit circle bundle $K_{1}^{-1} \subset K^{-1}$ with the property that the contraction with the rotational vector field $\partial / \partial \theta \in \operatorname{Vect}\left(K^{-1}\right)$ is 1 . The curvature is defined by

$$
\operatorname{curv}\left(\alpha_{X}\right) \in \Omega^{2}(X), \quad \pi^{*} \operatorname{curv}\left(\alpha_{X}\right)=\mathrm{d} \alpha_{X}
$$

where $\pi: K_{1}^{-1} \rightarrow X$ is the projection.

On the other hand, the given trivialization of $\phi^{*} K^{-1}$ defines a flat connection we denote $\alpha_{L}$. The difference between the two connection one-forms $\phi^{*} \alpha_{X}$ and $\alpha_{L}$ is a one-form on the base that we write $\phi^{*} \alpha_{X}-\alpha_{L} \in \Omega^{1}(L)$. The derivative of the difference oneform is the difference between the curvatures, which since $\alpha_{L}$ has trivial curvature is the pull-back of the curvature on $X$ :

$$
\mathrm{d}\left(\phi^{*} \alpha_{X}-\alpha_{L}\right)=\phi^{*}\left(\operatorname{curv}\left(\alpha_{X}\right)\right) .
$$

We include the following result of Cieliebak-Goldstein [18], see also Lotay-Pacini [42, Section 7] for convenience.

Lemma 2.1 For any Lagrangian immersion $\phi: L \rightarrow X$, the pair

$$
\left(\phi^{*} \alpha_{X}-\alpha_{L}, \operatorname{curv}\left(K^{-1}, \alpha_{X}\right)\right) \in \Omega^{2}(\phi)
$$

is a relative cocycle representing the Maslov class.

Proof The cocycle property is a consequence of the definition of the curvature (9) and flatness of $\alpha_{L}$. We show that the pair (10) represents the Maslov class. Let $u:(S, \partial S) \rightarrow(X, L)$ be a relative cycle. A trivialization $u^{*} K^{-1} \cong S \times \mathbb{C}$ induces a connection one-form denoted $\alpha_{S}$ on $u^{*} K^{-1}$. The Maslov index $I(u)$ is the integral of the difference of this connection with the connection one-form $(\partial u)^{*} \alpha_{L}$ induced by the trivialization $(\partial u)^{*} K^{-1} \cong \partial S \times \mathbb{C}$ : Let $\iota: \partial S \rightarrow S$ denote the inclusion. The difference between any two connection one-forms $(\partial u)^{*} \alpha_{L}, \iota^{*} \alpha_{S}$ on $(\partial u)^{*} K^{-1}$ is the pull-back of a one-form on the base $\partial S \cong S^{1}$, which we write (abusing notation) as $\alpha_{L}-\iota^{*} \alpha_{S}$. The Maslov index is

$$
\begin{aligned}
\int_{\partial S}(\partial u)^{*} \alpha_{L}-\iota^{*} \alpha_{S} & =\int_{\partial S}(\partial u)^{*} \alpha_{L}-(\partial u)^{*} \phi^{*} \alpha_{X}+\int_{\partial S}(\partial u)^{*} \phi^{*} \alpha_{X}-\iota^{*} \alpha_{S} \\
& =-\int_{\partial S}\left((\partial u)^{*} \phi^{*} \alpha_{X}-(\partial u)^{*} \alpha_{L}\right)+\int_{S} \operatorname{curv}\left(K^{-1}, \alpha_{X}\right)
\end{aligned}
$$

using Stokes' theorem and the fact that the curvature of $\alpha_{S}$ vanishes. This gives a special case of the pairing in (5). 
Maslov flows are isotopies of Lagrangians given by the above relative cocycle. Let $\omega_{t}$ be a family of symplectic forms and $\alpha_{t}$ be a family of connections on $K^{-1}$ satisfying

$$
\dot{\omega}_{t}=\operatorname{curv}\left(K^{-1}, \alpha_{t}\right) \text {. }
$$

Consider a family of immersions $\phi_{t}: L \rightarrow X$ satisfying

$$
\dot{\phi}_{t}:=\phi_{t}^{*} \omega_{t}\left(v_{t}, \cdot\right)=\phi^{*} \alpha_{t}-\alpha_{L}
$$

where

$$
v_{t}: L \rightarrow \phi_{t}^{*} T X / T L
$$

is the vector field determined by $\frac{d}{d t} \phi_{t}$. The family $\phi_{t}$ is Lagrangian if $\phi_{0}$ is. In order to give ourselves a bit more freedom, we allow ourselves a finite number of Hamiltonian isotopies in addition to the flow:

Definition 2.2 A Maslov flow for a family of symplectic forms $\omega_{t}, t \in(0, T)$ is a family of Lagrangian immersions

$$
\phi_{t, i}: L \rightarrow X, t \in\left[t_{i}, t_{i+1}\right]
$$

satisfying (11), except for a finite number of times

$$
t_{1}, \ldots, t_{k} \in(0, T)
$$

for which $\phi_{t_{i}, i}$ and $\phi_{t_{i}, i+1}$ are Hamiltonian isotopic:

$$
\phi_{t_{i}, i+1}=\phi_{t_{i}, i} \circ \psi_{H}
$$

where $H:[0,1] \times X$ is a time-dependent smooth function and $\psi_{H}: X \rightarrow X$ its Hamiltonian flow.

Example 2.3 (a) (Mean-curvature flow) Suppose that the symplectic manifold $(X, \omega)$ is equipped with a Kähler structure $J$, that is, an integrable almost complex structure $J: T X \rightarrow T X$ so that $\omega(\cdot, J \cdot)$ is a Riemannian metric. The mean curvature one-form is the unique one-form

$$
H_{\phi} \in \Omega^{1}(L)
$$

satisfying the following: Given a closed one-form $\beta \in \Omega^{1}(L)$, let $\phi_{t}: L \rightarrow$ $X, t \in(-\epsilon, \epsilon)$ be a variation of $\phi: L \rightarrow X$ corresponding to the one-form $\beta$ and denote by $\operatorname{Vol}\left(L, \phi_{t}\right)>0$ its volume with respect to the metric induced by pull-back of the Kähler metric on $X$. Then

$$
\frac{d}{d s} \operatorname{Vol}\left(L, \phi_{t}\right)=\int_{L}\left\langle\beta, H_{\phi}\right\rangle \mathrm{d} \operatorname{Vol}_{L}
$$


where $\langle\cdot, \cdot\rangle$ is the pairing between one-forms induced by the metric. In particular an immersion $\phi: L \rightarrow X$ represents a critical point of the volume if and only if the mean curvature one-form vanishes. The mean curvature one form $H_{\phi}$ is not closed but rather its derivative is the Ricci curvature $R_{\omega} \in \Omega^{2}(X)$ :

$$
\mathrm{d} H_{\phi}=\phi^{*} R_{\omega}
$$

by an observation of Smoczyk [52, Section 1.7] using the traced Codazzi equation.

Several other papers comment on the resulting coupled flow. By CieliebakGoldstein [18], Lotay-Pacini [42, Proposition 4.3], with respect to the trivialization of the anticanonical bundle over the Lagrangian, the mean curvature one-form is related to the difference of connections by

$$
H_{\phi}=(-1 / \pi)\left(\phi^{*} \alpha_{X}-\alpha_{L}\right) \in \Omega^{1}(L) .
$$

Hence any coupled mean-curvature/Kähler-Ricci flow is a reverse Maslov flow up to a rescaling of the time parameter by $1 / \pi$. The same paper [42, Section 7] proves short-time existence of the coupled flow.

(b) (Unions) Let $\phi_{t, 0}: L_{0} \rightarrow X$ and $\phi_{t, 1}: L_{1} \rightarrow X$ be Maslov flows. Then the disjoint union

$$
\phi_{t, 0} \sqcup \phi_{t, 1}: L_{0} \sqcup L_{1} \rightarrow X
$$

is also a Maslov flow. Indeed, the mean curvature on the union restricts to $H_{\phi_{t, k}}$ on $L_{k}, k \in\{0,1\}$.

(c) (Products) Let $\phi_{t, 0}: L_{0} \rightarrow X_{0}$ and $\phi_{t, 1}: L_{1} \rightarrow X_{1}$ be Maslov flows. Then the product

$$
\phi_{t, 0} \times \phi_{t, 1}: L_{0} \times L_{1} \rightarrow X_{0} \times X_{1}
$$

is also a Maslov flow. Indeed, the mean curvature on the product $H_{\phi_{t, 0} \times \phi_{t, 1}} \in$ $\Omega^{1}\left(L_{0} \times L_{1}\right)$ is the sum of pull-backs of $H_{\phi_{t, k}}$ on $L_{k}, k \in\{0,1\}$.

The following are elementary examples of Maslov flow:

Example 2.4 (a) (Linear case) Let $X=\mathbb{R}^{2 n}$ and $L=\mathbb{R}^{n}$ with immersion $l \rightarrow$ $(l, 0)$. Then the constant isotopy

$$
\phi_{s}: L \rightarrow X, q \mapsto(q, 0)
$$

is a Maslov flow. Indeed the standard trivialization $K^{-1}=X \times \mathbb{C}$ agrees with the trivialization $\phi_{s}^{*} K^{-1} \cong L \times \mathbb{C}$, and so the connection one-form $\alpha_{s} \in \Omega^{1}\left(K_{1}^{-1}\right)$ (and the mean curvature) vanishes. 
(b) (Circle case) Consider the embedding of the circle of radius 1

$$
\phi: L=\left\{q^{2}+p^{2}=1\right\} \rightarrow \mathbb{R}^{2}=X
$$

Let $\theta: L \rightarrow S^{1}$ denote the angular coordinate. The trivialization of $\phi^{*} K^{-1}$ induced by the Lagrangian is that induced by the section $e^{i \theta} \partial_{x}$ and so is related to the standard trivialization by multiplication by $e^{i \theta}$. Thus the connection one-form of the connection with respect to the trivialization of $\phi^{*} K^{-1}$ is

$$
\phi^{*} \alpha_{X}-\alpha_{L}=\mathrm{d} \theta \in \Omega^{1}(L) .
$$

Writing the standard symplectic form $\omega=\mathrm{d} q \wedge \mathrm{d} p$ on $X$ as $\omega=r \mathrm{~d} r \wedge \mathrm{d} \theta$, the corresponding vector field is the outward normal $v_{t}=\partial_{r} / r \in \operatorname{Vect}(L)$ (corresponding to the fact that the mean curvature $H_{\phi}$ of a circle $\phi: S^{1} \rightarrow \mathbb{R}^{2}$ is inversely proportional to its radius). Thus a Maslov flow $\phi_{t}: L \rightarrow X$ is given by outward flow at speed $1 / r$ with $r=1$ at $t=0$ :

$$
\phi_{t}: L \rightarrow X, \quad(q, p) \mapsto(2 t+1)^{1 / 2}(q, p) .
$$

Thus in particular the area enclosed by the immersion is $\pi(1+2 t)$ and increases linearly with $t$.

(c) (Local model for self-tangencies) Let $L$ denote the union of $S^{1} \times i \mathbb{R}^{n-1}$ and $\{1\} \times \mathbb{R}^{n-1}$. By the disjoint union and product axioms (b) and (c) above, a Maslov flow is given by

$$
\phi_{t}\left(z_{1}, \ldots, z_{n}\right)=\left\{\begin{array}{ll}
\left((1+2 t)^{1 / 2} z_{1}, z_{2}, \ldots, z_{n}\right) & z_{1} \in S^{1} \\
\left(z_{1}, z_{2}, \ldots, z_{n}\right) & z_{1} \in 1+i \mathbb{R}
\end{array} .\right.
$$

In order to obtain the invariance of Floer cohomology we will assume that our flow is in some sense generic.

Definition 2.5 By a generic Maslov flow we mean a flow $\phi_{t}$ defined by a generic choice of connection one forms $\alpha_{t}$ with respect to the $C^{\infty}$ topology. For $H \in C^{l}([0, T] \times X)$ a change in the connection one-form $\alpha_{t}$ on $K^{-1}$ by the pull-back of $\mathrm{d} H_{t}$ changes the Maslov flow equation to

$$
\dot{\phi}_{t}=\alpha_{t}-\phi_{t}^{*} \alpha_{L}+\phi_{t}^{*} \mathrm{~d} H_{t} .
$$

Thus a generic change in the connection one-form is essentially the same as a generic Hamiltonian perturbation.

The following is standard from properties of generic homotopies of functions in Cerf [11], see also Sullivan [56, 3.12]. 
Theorem 2.6 Let $\phi_{t}: L \rightarrow X, t \in[0,1]$ be a Maslov flow such that $\phi_{0}$ has only transverse self-intersections. There exists an open dense set of Hamiltonian perturbations $C^{\infty}([0, T] \times X)^{\text {reg }}$ with the property that for $H \in C^{\infty}([0, T] \times X)^{\text {reg }}$, the $H$-perturbed flows $\phi_{t}: L \rightarrow X, t \in[0,1]$ have the property that the self-intersections $\left(\phi_{t} \times \phi_{t}\right)^{-1}\left(\Delta_{L}\right)-\Delta_{L}$ of $\phi_{t}$ are transverse except for all but finitely many values of $t$ for which there is a single self-intersection $\left(x_{-}, x_{+}\right) \in L, \phi_{t}\left(x_{-}\right)=\phi_{t}\left(x_{+}\right)$that has a non-degenerate quadratic tangency.

Proof The proof is a Sard-Smale argument applied to a universal moduli space of intersection points. Let $C^{l}([0, T] \times X)$ denote the space of time-dependent functions of class $C^{l}$. Let

$$
\operatorname{Lag}(L, X)_{l}:=\left\{\begin{array}{l|l}
\phi \in \operatorname{Map}([0, T] \times L, X)_{l} \mid \begin{array}{l}
\operatorname{ker}(D \phi)=\{0\}, \phi_{0}=\phi(0) \\
\phi^{*} \omega=0 \forall t \in[0, T]
\end{array}
\end{array}\right\}
$$

denote the space of time-dependent Lagrangian immersions from $L$ to $X$ of class $C^{l}$. Consider the universal space of self-intersections

$$
\mathcal{M}^{\text {univ }, s i}:=\left\{\begin{array}{l}
\left(x_{1}, x_{2}, t_{0}, H, \phi\right) \in L \times L \times[0,1] \times C^{l}([0, T] \times X) \times \operatorname{Lag}(L, X)_{l} \\
\dot{\phi}_{t}=\alpha_{t}+\mathrm{d} \phi_{t}^{*} H_{t}, \quad \phi_{t_{0}}\left(x_{1}\right)=\phi_{t_{0}}\left(x_{2}\right)
\end{array}\right\} .
$$

The universal moduli space (13) is a smooth Banach manifold by an application of the implicit function theorem, as follows. First, variations in $H_{t}$ near $t=0, x=x_{1}$ span the space of normal vector fields to $\phi_{0}$ near $x_{1}$. Indeed, let $H \in C^{\infty}(X)$ and $\varkappa \in C^{\infty}([0, T])$ supported and equal to 1 near 0 a cutoff function. The Maslov flow $\phi_{t}^{\prime}: L \rightarrow X$ defined by the connection

$$
\alpha+\pi^{*}(1 / \epsilon) \mathrm{d} H(\epsilon t) \in \Omega^{1}\left(K_{1}^{-1}\right)
$$

converges as $\epsilon \rightarrow 0$ to the composition of the restriction to $L$ of the Hamiltonian flow $\psi_{t}: X \rightarrow X$ of $H$ with the original Maslov flow $\phi_{t}: L \rightarrow X$. It follows that the linearization of the condition $\phi_{t_{0}}\left(x_{1}\right)=\phi_{t_{0}}\left(x_{2}\right)$ is surjective.

From the universal moduli space we obtain a comeager set of regular values for the projection. By the Sard-Smale theorem for $l \geq 1$ the projection

$$
\pi: \mathcal{M}^{\mathrm{univ}, s i} \rightarrow C^{l}([0, T] \times X)
$$

has a comeager set of regular values $C^{l}([0, T] \times X)^{\text {reg }}$. For such regular $H \in C^{l}([0, T] \times$ $X)^{\text {reg }}$, the projection of $\pi^{-1}(H)$ onto the fiber $[0,1]$ has open and comeager set of regular values $[0,1]^{\text {reg }}$. Since $L$ is compact, the set of irregular values $[0,1] \backslash[0,1]^{\text {reg }}$ is discrete, hence finite. Thus for regular $H$ the set of times $t$ for which the intersection 
$L \times{ }_{\phi_{t}} L$ is not transverse is finite. Since the space of smooth elements of this comeager set is also open and dense, the claim follows.

Next we show that for generic Hamiltonian perturbations the non-transverse intersections are non-degenerate. Consider the universal space of tangencies

$$
\mathcal{M}^{\text {univ }, t}:=\left\{\begin{array}{l}
\left(v_{1}, v_{2}, H, \phi\right) \in T L \times T L \times[0,1] \times C^{l}([0, T] \times X) \times \operatorname{Lag}(L, X)_{l} \mid \\
\dot{\phi}_{t}=\alpha_{t}+\mathrm{d} \phi_{t}^{*} H_{t}, D \phi_{t_{0}}\left(v_{1}\right)=D \phi_{t_{0}}\left(v_{2}\right) \neq 0
\end{array}\right\}
$$

is cut out transversally, since the linearization of $D \phi_{t}$ is the second derivative of $\phi_{t}$ and a Hamiltonian variation at $x_{1}, x_{2}$ at time 0 produces an arbitrary variation this derivative at time $t_{0}$. Let $C^{l}([0, T] \times X)^{\text {reg, }}$ ' denote the set of regular values for the projection $\pi$ onto $C^{l}([0, T] \times X)$ For $H \in C^{l}([0, T] \times X)^{\text {reg, }}{ }^{\prime}$ the intersection $D_{t} \phi\left(T_{x_{1}} L\right) \cap D_{t} \phi\left(T_{x_{2}} L\right)$ is trivial except for finitely many times $t=t_{1}, \ldots, t_{k}$ at which the intersection is one-dimensional. So there is a point of tangency $\left(x_{i,-}, x_{i,+}\right) \in L \times_{\phi_{t_{i}}} L$ and for these intersections the tangency is quadratic.

A final argument using a universal space of simultaneous self-tangencies

$\mathcal{M}^{\text {univ }, s s t}:=\left\{\begin{array}{l}\left(v_{1}, v_{2}, v_{3}, v_{4}, t, H, \phi\right) \in T L^{4} \times[0,1] \times C^{l}([0, T] \times X) \times \operatorname{Lag}(L, X)_{l} \mid \\ \dot{\phi}_{t}=\alpha_{t}+\mathrm{d} \phi_{t}^{*} H_{t}, D \phi_{t}\left(v_{1}\right)=D \phi_{t}\left(v_{2}\right), D \phi_{t}\left(v_{3}\right)=D \phi_{t}\left(v_{4}\right) \\ \left\{\mathbb{R} v_{1}, \mathbb{R} v_{2}, \mathbb{R} v_{3}, \mathbb{R} v_{4}\right\} \text { distinct, non-zero }\end{array}\right\}$

guarantees that at most one tangency occurs at each non-transverse time.

Definition 2.7 (a) (Self-tangency moves) A Maslov flow $\phi_{t}: L \rightarrow X$ undergoes $a$ self-tangency move at $p \in X$ and time $t=0$ if the following holds: There exist Darboux coordinates $\left(x_{1}, y_{1}, \ldots, x_{n}, y_{n}\right)$ on a contractible open neighborhood $U$ of $p$ such that the intersection $U \cap \phi_{s}(L)$ is the union of Lagrangians $L_{1}$ and $L_{2}(t)$ where

$$
L_{1}=L_{1}^{\prime} \times L_{1}^{\prime \prime}, \quad L_{2}(t)=L_{2}^{\prime}(t) \times L_{2}^{\prime \prime}
$$

for some Lagrangians

$$
L_{1}^{\prime}, L_{2}^{\prime}(t) \subset \mathbb{C}, \quad L_{1}^{\prime \prime}, L_{2}^{\prime \prime} \subset \mathbb{C}^{n-1}
$$

where

$$
L_{1}^{\prime} \subset\left\{y_{1}=0\right\}, \quad L_{2}^{\prime}(t) \subset\left\{x_{1}^{2}+\left(y_{1}-1\right)^{2}=(1+2 t)^{2}\right\}
$$

are subarcs of the straight line and the circle in the $z_{1}$ plane, respectively, and the factors $L_{1}^{\prime \prime}$ and $L_{2}^{\prime \prime}$ are linear Lagrangians in $\mathbb{C}^{n-1}=\left\{z_{1}=0\right\}$ intersecting transversally at 0 . The mean curvature to a circle $L_{2}(t)^{\prime}$ is the outward normal while the mean curvatures for the linear spaces are trivial. Hence the isotopy $\phi_{t}$ is a Maslov flow as in Example 2.4. 
(b) (Admissible flows) Let $\phi_{t}: L \rightarrow X$ be a Maslov flow of Lagrangian immersions with self-tangency instants $t_{1}, \ldots, t_{k} \in(0, T)$ at points $x_{1}, \ldots, x_{k} \in X$. Say that $\phi_{t}: L \rightarrow X, s \in[0, T]$ is an admissible family of immersions if there exist small disjoint intervals $\left(t_{j}-\delta, t_{j}+\delta\right) \subset[0, T]$ such that all self-tangency instants $t_{j}$ are standard in the sense that $\phi_{t}$ near $t_{j}$ is given by the local model (14), and in addition any self-tangency occurs at a rational time $t \in(0, T) \cap \mathbb{Q}$.

The rationality of the time will be used in Section 9 to define the Floer theory in a neighborhood of the singular time.

Lemma 2.8 (Similar to Lemma 3.6 of [23]) Let $\phi_{t}: L \rightarrow X, t \in\left[t_{0}, t_{1}\right]$ be a Maslov flow of Lagrangian immersions connecting $\phi_{t_{0}}$ to $\phi_{t_{1}}$. Then for any $\epsilon$ there is an admissible Maslov flow $\phi_{t}^{\prime}$ of Lagrangian immersions $\epsilon$-close to $\phi_{t}$ connecting $\phi_{t_{0}}$ to $\phi_{t_{1}}$.

Proof First we perturb to make the flow generic. Locally the family of immersions $\phi_{t}: L \rightarrow X$ is given by a family of closed one-forms $\alpha_{t} \in \Omega^{1}(L)$ as in (7). For each self-tangency instant $t_{j}$, there exist points $x_{j}^{-}, x_{j}^{+} \in L$ with $\phi\left(x_{j}^{+}\right)=\phi\left(x_{j}^{-}\right)=x_{j}$ such that

$$
D \phi_{t_{j}}\left(x_{j}^{-}\right)\left(T_{x_{j}^{-}} L\right) \cap D \phi_{t_{j}}\left(x_{j}^{+}\right)\left(T_{x_{j}^{+}} L\right) \neq\{0\} .
$$

Using the Sard-Smale theorem one checks that for a dense open subset of Hamiltonian perturbations $H_{t}$ as in (12), the equation cuts out the solutions $\left(x_{j}^{-}, x_{j}^{+}\right)$transversally. Equivalently,

$$
\operatorname{dim} I_{j}=1, \quad I_{j}:=D \phi_{t_{j}}\left(x_{j}^{-}\right)\left(T_{x_{j}^{-}} L\right) \cap D \phi_{t_{j}}\left(x_{j}^{+}\right)\left(T_{x_{j}^{+}} L\right)
$$

and the tangency of the components of $\phi_{t_{j}}^{-1}\left(I_{j}\right)$ is quadratic.

Having achieved a quadratic tangency, we apply a Hamiltonian isotopy to make the Lagrangians of standard form just before the isotopy. We suppose that the self-tangency represents the death of two self-intersection points; the birth case is easier. By changing the cubic and higher order terms in the generating functions for the Lagrangians locally we obtain an isotopy $L_{12}(t)$ between $L_{1}\left(t_{j}-\delta\right) \cup L_{2}\left(t_{j}-\delta\right)$ and $L_{1}^{\text {st }} \cup L_{2}^{\text {st }}(t)$ where $L_{2}^{\text {st }}(t)$ is a circle of radius $r(t)$ depending on $t$. As in Moser's proof of the Darboux lemma, there exists a diffeomorphism $\psi$ between neighborhoods $U_{0}, U_{1}$ of 0 in $\mathbb{C}^{n}$ taking $L_{1}(t) \cup L_{2}(t)$ to $L_{1}^{\text {st }} \cup L_{2}^{\text {st }}(t)$. Let $\omega_{0} \in \Omega^{2}\left(U_{0}\right)$ be the standard symplectic form, and $\omega_{1}=\psi^{*} \omega_{0} \in \Omega^{2}\left(U_{1}\right)$ the pull-back. Let

$$
\omega_{t}=(1-t) \omega_{0}+t \omega_{1} \in \Omega^{2}\left(U_{1}\right)
$$


In order to correct $\psi$ to a symplectomorphism note that $\frac{d}{d t} \omega_{t}$ is exact if $\frac{d}{d t} A_{t}=0$ where $A_{t}=\int_{S} u^{*} \omega_{t}$ is the area of the disk $u: S \rightarrow \mathbb{C}^{n}$ whose boundary from 1 to -1 resp. -1 to 1 lies in $L_{1}^{\text {st }}$ resp. $L_{2}^{\text {st }}$ connecting the two self-intersection points. By choosing the parameter $r(t)$ and the isotopy suitably, we may ensure that this area $A_{t}$ is constant in the isotopy $L_{12}(t)$; then Moser's argument produces the desired symplectomorphism equal to the identity on $L_{1}^{\mathrm{st}} \cup L_{2}^{\mathrm{st}}(t)$.

The flows of the original Lagrangian immersion and the flow in the standard model may be combined using a cutoff function. In the local model, the flow of $L_{1}(t)$ is given by the graph of the differential $\mathrm{d} H_{1}(t)$ of a time-dependent Hamiltonian $H_{1}(t): L_{1}^{\mathrm{st}} \rightarrow \mathbb{R}$, while $L_{2}(t)$ is the graph of $\mathrm{d} H_{2}(t)$ for some function $H_{2}: L_{2}^{\text {st }}(t) \rightarrow \mathbb{R}$. Finally, by choosing a generic complement to $T_{0} L_{1}^{\mathrm{st}}$, we may assume that $L_{2}^{\mathrm{st}}(t)$ is the graph of $\mathrm{d} H_{12}(t)$ for some $H_{12}(t): L_{1}^{\mathrm{st}} \rightarrow \mathbb{R}$, By choosing $U$ sufficiently small, we may assume that $\mathrm{d} H_{1}(t), \mathrm{d} H_{2}(t)$ are small in comparison with $\mathrm{d} H_{12}(t)$, since $\mathrm{d} H_{12}(t)$ has at worst quadratic vanishing at $(q, p)=0$ and $t=0$, and $\mathrm{d} H_{2}(t), \mathrm{d} H_{1}(t)$ vanish to third order. Let $\varkappa$ be a cutoff function vanishing in a neighborhood of 0 . The flow $\phi_{t}^{\prime}: L \rightarrow X$ obtained by replacing $L_{1}(t), L_{2}(t)$ by the graphs of $\mathrm{d}\left(\varkappa H_{1}(t)\right), \mathrm{d}\left(\varkappa H_{2}(t)\right)$ have the same self-intersections, and are equal to the standard flow in a neighborhood of 0 . By (12), $\phi_{t}^{\prime}$ is also a Maslov flow, and is of standard form near the tangency.

At the end time of the combined flow, the two nearby self-intersection points have disappeared, and so the resulting Lagrangians are disjoint and equal outside of a small ball. In particular, the flow produces an exact deformation from $L_{1}^{\prime}\left(t_{j}+\delta\right) \cup L_{2}^{\prime}\left(t_{j}+\delta\right)$ to $L_{1}\left(t_{j}+\delta\right) \cup L_{2}\left(t_{j}+\delta\right)$ that is the identity outside of a small ball, and a standard argument implies that this isotopy is achievable by a Hamiltonian flow. Thus, changing the flow by a Hamiltonian isotopy at $t_{j}+\delta$ produces a piece-wise smooth flow that matches up with the original flow for $t \geq t_{j}+\delta$.

Finally, the time of the self-tangency can be perturbed an arbitrary small amount using a Hamiltonian isotopy, so that the times $t_{j}$ of the self-tangencies $D \phi_{t_{j}}\left(T_{x_{1}} L\right) \cap$ $D \phi_{t_{j}}\left(T_{x_{2}} L\right) \neq\{0\}$ become rational.

\section{Holomorphic disks with self-transverse boundary condi- tion}

The Morse model of Floer theory counts treed pseudoholomorphic disks with Lagrangian boundary condition. First we recall basic terminology regarding stable disks. A disk will mean a 2-manifold-with-boundary $S_{\circ}$ equipped with a complex structure $j_{S_{\circ}}: T S_{\circ} \rightarrow T S_{\circ}$ so that the surface $S_{\circ}$ is biholomorphic to the closed unit disk 


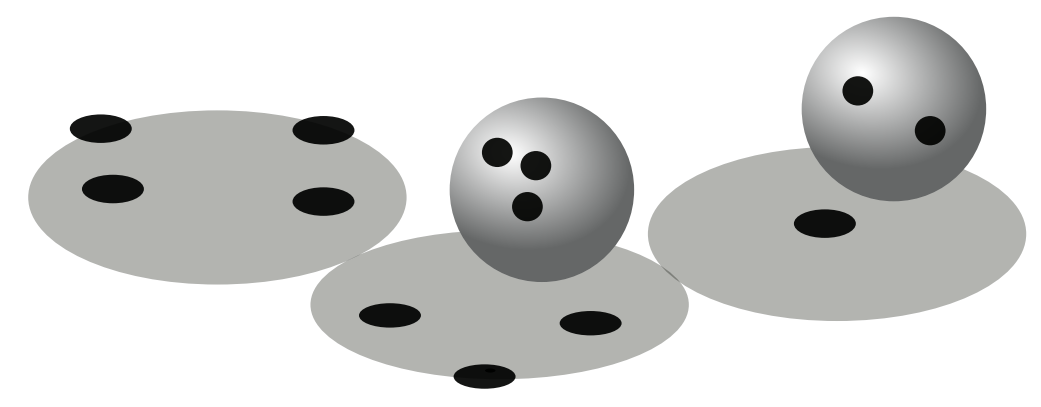

Figure 4: A stable disk with boundary and interior markings

$\{z \in \mathbb{C}|| z \mid \leq 1\}$. A sphere will mean a complex one-manifold $S_{\bullet}$ biholomorphic to the complex projective line $\mathbb{P}^{1}=\left\{\left[\zeta_{0}: \zeta_{1}\right] \mid \zeta_{0}, \zeta_{1} \in \mathbb{C}\right\}$. A nodal disk $S$ is a union

$$
S=\left(\bigcup_{i=1}^{n_{\circ}} S_{\circ, i}\right) \cup\left(\bigcup_{i=1}^{n_{\bullet}} S_{\bullet, i}\right) / \sim
$$

of a finite number of disks $S_{\circ, i}, i=1, \ldots, n_{\circ}$ and spheres $S_{\bullet, i}, i=1, \ldots, n_{\bullet}$ identified at pairs of distinct points called nodes $w_{1}, \ldots, w_{m}$. Each node

$$
w_{k}=\left(w_{k}^{-}, w_{k}^{+}\right) \in S_{i_{-}(k)} \times S_{i_{+}(k)}
$$

is a pair of distinct points $w_{k}^{ \pm} \in S_{i_{ \pm}(k)}$ where $S_{i_{ \pm}(k)}$ are the (disk or sphere) components of $S$ adjacent to the node $w_{k}$; the resulting topological space $S$ is required to be simplyconnected, see Figure 3 (produced using Inkscape and [62].) The complex structures on the disks and spheres induce a complex structure on the tangent bundle TS (which is a vector bundle except at the nodal points) denoted $j: T S \rightarrow T S$. A boundary resp. interior marking of a nodal disk $S$ is an ordered collection of non-nodal points $\underline{z}=\left(z_{0}, \ldots, z_{d}\right) \in \partial S^{d+1}$ resp. $\underline{z}^{\prime}=\left(z_{1}^{\prime}, \ldots, z_{c}^{\prime}\right) \in \operatorname{int}(S)^{c}$ on the boundary resp. interior, whose ordering is compatible with the orientation on the boundary $\partial S$ in the case of boundary markings. The combinatorial type is the graph

$$
\Gamma(S)=(\operatorname{Vert}(\Gamma(S)), \operatorname{Edge}(\Gamma(S):(h \times t): \operatorname{Edge}(\Gamma(S)) \rightarrow \operatorname{Vert}(\Gamma(S)) \cup\{\infty\}
$$

obtained by setting $\operatorname{Vert}(\Gamma(S))$ to be the set of disk and sphere components in $S$ and $\operatorname{Edge}(\Gamma(S))$ the set of nodes $w_{1}, \ldots, w_{m}$ (each connected to the vertices corresponding to the disks or spheres they connect) and markings $z_{i, \bullet}, z_{j, \circ}$ (each connecting the corresponding vertex to infinity); the graph $\Gamma(S)$ is required to be a tree, that is, connected with no cycles among the combinatorially finite edges. The set of edges is naturally equipped with a partition into subsets

$$
\operatorname{Edge}(\Gamma(S))=\text { Edge }_{\bullet}(\Gamma(S)) \cup \operatorname{Edge}_{\circ}(\Gamma(S))
$$


corresponding to interior resp. boundary markings and nodes, respectively. The set of boundary edges $h^{-1}(v) \cup t^{-1}(v) \cap \operatorname{Edge}_{\circ}(\Gamma(S))$ meeting some vertex $v \in \operatorname{Vert}(\Gamma(S))$ is naturally equipped with a cyclic ordering giving $\Gamma(S)$ the partial structure of a ribbon graph. The set of boundary and interior semi-infinite leaves

$$
\begin{aligned}
\text { Edge }_{\circ, \rightarrow}(\Gamma(S)) & :=\text { Edge }_{\circ}(\Gamma(S)) \cap \text { Edge }_{\rightarrow}(\Gamma(S)), \\
\text { Edge }_{\bullet} \rightarrow(\Gamma(S)) & :=\text { Edge }_{\bullet}(\Gamma(S)) \cap \text { Edge }_{\rightarrow}(\Gamma(S)), \\
\text { Edge }_{\rightarrow}(\Gamma(S)) & :=h^{-1}(\infty) \cup t^{-1}(\infty)
\end{aligned}
$$

is each equipped with an ordering; these orderings will be omitted from the notation to save space. We call the leaves corresponding to interior resp. boundary markings bulk resp. boundary leaves, for lack of better terminology. A marked disk $\left(S, \underline{z}, \underline{z}^{\prime}\right)$ is stable if it admits no automorphisms preserving the markings. The moduli space of stable disks with fixed number of boundary markings and no interior markings admits a natural structure of a cell complex which identifies the moduli space with Stasheff's associahedron.

Treed disks are defined by replacing nodes with broken segments as in the pearly trajectories of Cornea-Lalonde [20], Biran-Cornea [8], and also Seidel [55]. By a segment we will mean an oriented connected Riemannian manifold $T$, necessarily isometric with a closed sub-interval of the real line $\left[t_{-}, t_{+}\right] \cap \mathbb{R}, t_{ \pm} \in\{-\infty\} \cup \mathbb{R} \cup\{\infty\}$. A broken segment $T$ is obtained from a finite collection of segments $T_{1}, T_{2}$ with an infinite positive resp. negative end by $T=T_{1} \cup\{\infty\} \cup T_{2}$ where $\{\infty\}=\overline{T_{1}} \cap \overline{T_{2}}$ is the point of breaking, with the topology that makes $T$ also a closed interval. The metrics on the open subsets $T_{1}, T_{2}$ are given as part of the data; that is, any broken segment is equipped with a metric on the complement of the finite set of breaking points. A treed disk is a space $C$ obtained from a nodal disk $S$ by replacing each boundary node or boundary marking corresponding to an edge $e \in \Gamma(S)$ with a broken segment $T_{e}$; we also allow the case $C \cong \mathbb{R}$ with a single edge infinite in both directions in which case the underlying "disk" is empty. ${ }^{3}$ Each broken line is obtained from a sequence of possibly infinite closed real intervals by gluing together endpoints, and is equipped with a length and number of breakings

$$
\ell(e) \in[0, \infty], \quad b(e) \in \mathbb{Z}_{\geq 0}
$$

where the length $\ell(e)$ is equal to infinity by assumption if the segment $T_{e}$ replaces a marking $z_{e} \in S$. For the combinatorially finite edges, $\ell(e)=\infty$ if and only if the number of breakings $b(e)>0$. Denote by $T \subset C$ the union of (finite or infinite length)

\footnotetext{
${ }^{3}$ More generally one could also replace interior nodes and markings with segments, but since in this case we will rule out sphere bubbling there is no need for the most general construction.
} 


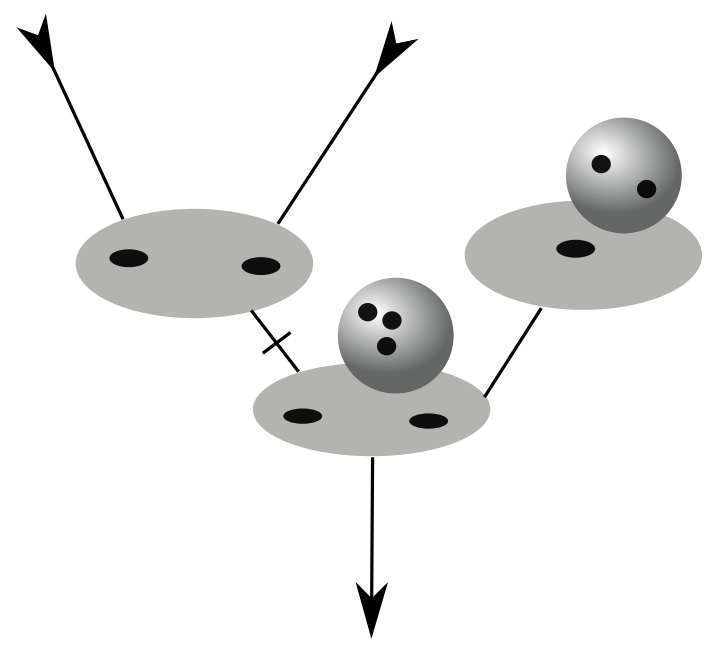

Figure 5: A treed disk with $d=2$ incoming edges

segments so produced. Then $C=S \cup T$ where the one-dimensional part $T$ is joined to the two-dimensional part $S$ at a finite set of points on the boundary of $S$, which we call the nodes of the treed disk (as they correspond to the nodes in the underlying nodal disk.) The semi-infinite edges in the one-dimensional part $T$ are oriented by requiring that the first boundary marking is outgoing while the remaining semi-infinite edges are incoming; the outgoing semi-infinite edge is referred to as the root while the other semi-infinite edges are leaves. On each segment $T_{e} \subset T$ corresponding to an edge Edge $(\Gamma(S))$ we assume the existence of a coordinate $s$ that identifies $T_{e}$ with the interval $[0, \ell(e)]$; this coordinate will be used in the construction of gradient trees below. The combinatorial type $\Gamma(C)=(\operatorname{Vert}(C), \operatorname{Edge}(C))$ of a broken tree $C$ is defined similarly to that for broken disks but now the set of edges $\operatorname{Edge}(C)$ is equipped with a partition $\operatorname{Edge}(C)=\operatorname{Edge}_{0}(C) \cup \operatorname{Edge}_{(0, \infty)}(C) \cup \operatorname{Edge}_{\infty}(C)$ corresponding to whether the length is zero, finite and non-zero, or infinite, and equipped with a labelling by the number of breakings. A treed disk is stable if the underlying disk is stable, each combinatorial finite edge has at most one breaking, and each semi-infinite edge is unbroken. An example of a treed disk with one broken edge (indicated by a small hash through the edge) is shown in Figure 3.

The stability condition gives a compact, Hausdorff moduli space with a universal curve. For a given combinatorial type $\Gamma$ denote by $\mathcal{M}_{\Gamma}$ the moduli space of treed disks of combinatorial type $\Gamma$ and $\overline{\mathcal{M}}_{d}=\cup_{\Gamma} \mathcal{M}_{\Gamma}$ the union over combinatorial types $\Gamma$ with $d$ incoming semi-infinite edges. The moduli space $\overline{\mathcal{M}}_{d}$ is compact with a universal curve 


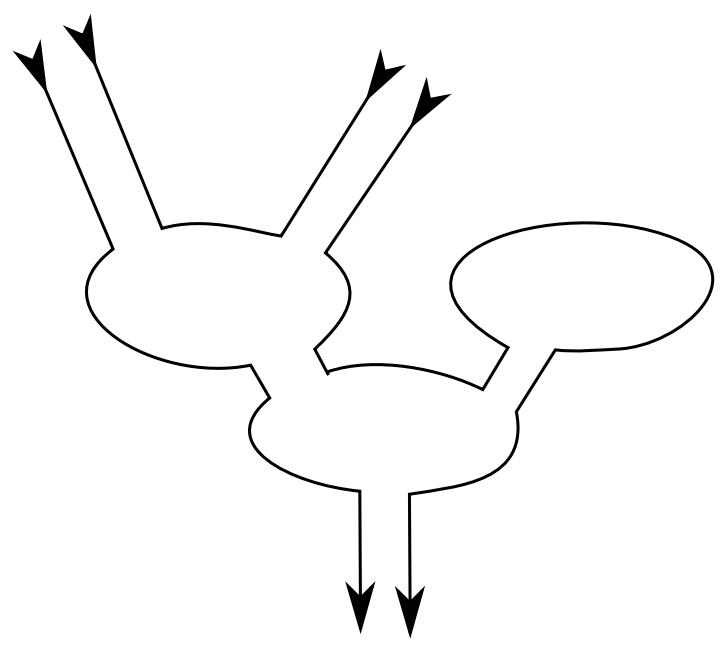

Figure 6: Boundary of a treed disk with $d=2$ incoming edges

$\overline{\mathcal{U}}_{d}$ given as the space of isomorphism classes of pairs $[C, z]$ where $C$ is a holomorphic treed disk and $z \in C$ is a point in the one or two dimensional locus. Depending on which is the case one has a splitting

$$
\overline{\mathcal{U}}_{d}=\overline{\mathcal{S}}_{d} \cup \overline{\mathcal{T}}_{d}
$$

of the universal treed disk into one-dimensional and two-dimensional parts $\overline{\mathcal{T}}_{d}$ resp. $\overline{\mathcal{S}}_{d}$. That is, $\overline{\mathcal{U}}_{d}=\overline{\mathcal{T}}_{d} \cup \overline{\mathcal{S}}_{d}$ where the fibers of $\overline{\mathcal{T}}_{d} \rightarrow \overline{\mathcal{M}}_{d}$ resp. $\overline{\mathcal{S}}_{d} \rightarrow \overline{\mathcal{M}}_{d}$ are one resp. two-dimensional. We denote by $\overline{\mathcal{S}}_{\Gamma}, \overline{\mathcal{T}}_{\Gamma}$ the parts of the universal treed disk living over $\overline{\mathcal{M}}_{\Gamma}$.

Holomorphic treed disks for immersed Lagrangians are defined as in the embedded case, but requiring a double cover of the tree parts to obtain the boundary lift. Given a treed disk $C$ we define a one-manifold $\partial C$ by gluing together the boundary of each disk $S_{v}, v \in \operatorname{Vert}(\Gamma)$ minus the points $T_{e} \cap S, e \in \operatorname{Edge}(\Gamma)$ where edges attach with $t w o$ copies of each edge $T_{e}$ as in Figure 6.

To define holomorphic treed disks, we make a choice of almost complex structure and Morse function.

(a) Let $J: T X \rightarrow T X$ be an almost complex structure taming the symplectic form $\omega \in \Omega^{2}(X)$; such a choice is unique up to isotopy. Later, for the purposes of constructing Donaldson hypersurfaces we will assume that $J$ is compatible with $\omega$ in the sense that $\omega(\cdot, J \cdot)$ is a Riemannian metric. 
(b) Let $m: L \rightarrow \mathbb{R}$ be a Morse function. Given a metric on $L$ we obtain a gradient vector field and time $t$ flow

$$
\operatorname{grad}(m) \in \operatorname{Vect}(L), \quad \psi_{t} \in \operatorname{Diff}(L) .
$$

For any critical point $x \in \operatorname{crit}(m)$ we have stable and unstable manifolds

$$
W_{x}^{ \pm}=\left\{\begin{array}{l|l}
y \in L & \lim _{t \rightarrow \pm \infty} \psi_{t}(y)=x
\end{array}\right\} .
$$

We assume that the Morse function and metric are Morse-Smale in the sense that the unstable and stable manifolds intersect transversally:

$$
W_{x}^{+} \pitchfork W_{y}^{-}, \quad \forall x, y \in \operatorname{crit}(m) .
$$

Thus in particular the moduli space of Morse trajectories

$$
\mathcal{M}(x, y) \cong\left(W_{x}^{+} \cap W_{y}^{-}\right) / \mathbb{R}
$$

is smooth for any $x, y \in \operatorname{crit}(m)$; these are special cases of moduli spaces of holomorphic treed disks, namely the case that the number of disks in the configuration is zero. Choose coordinates $s$ on each segment of $T$ so that each segment has the given length. We extend $m$ to the space of self-intersection points $L \times_{\phi} L$ by extension by zero, so that any gradient trajectory $u: T_{e}^{2} \rightarrow L \times_{\phi} L$ in $L \times_{\phi} L$ is constant.

Definition 3.1 A holomorphic treed disk with boundary in $\phi: L \rightarrow X$ consists of a treed disk $C=S \cup T$ and a pair of continuous maps

$$
u: C \rightarrow X, \quad \partial u: \partial C \rightarrow L
$$

satisfying the conditions that the map is a pseudoholomorphic map on the surface part, gradient trajectory on the tree parts, and the map $\partial u$ is a lift to $L$ of the restriction of $u$ to the boundary of $S$ :

$$
\begin{aligned}
J \mathrm{~d}_{H}\left(\left.u\right|_{S}\right) & =\mathrm{d}_{H}\left(\left.u\right|_{S}\right) j \\
-\operatorname{grad}(m)\left(\left.\partial u\right|_{T}\right) & =\frac{d}{d s}\left(\left.\partial u\right|_{T}\right) \\
u \mid \partial C & =\phi \circ \partial u
\end{aligned}
$$

where $\mathrm{d}_{H} u=\mathrm{d} u-H(u)$.

Note that the one-dimensional part $T$ of the domain $C$ is equipped with a decomposition $T=T_{2} \cup T_{1}$, so that $u \mid T_{2}$ takes values in the finite set of self-intersections

$$
L \times_{\phi} L-\Delta_{L}, \quad \Delta_{L}=\{(l, l) \mid l \in L\}
$$


and $u \mid T_{1}$ is a map to $L$. We call $T_{1}$ resp. $T_{2}$ the unbranched resp. branched one-dimensional locus.

The combinatorial data of a treed holomorphic disk is packaged into a labelled graph called the combinatorial type: for a pseudoholomorphic treed disks $u: C \rightarrow X$ is the type is the combinatorial type $\Gamma$ of the underlying treed disk $C$ together with the labelling of vertices $v \in \operatorname{Vert}(\Gamma)$ corresponding to sphere and disk components $S_{v}, v \in \operatorname{Vert}(\Gamma)$ by their (relative) homology classes

$$
d(v) \in H_{2}(X) \cup H_{2}(\phi)
$$

and the labelling

$$
t(e) \in\{1,2\}
$$

of edges by their branch type (whether they map to $\Delta_{L}$ or to $L \times_{\phi} L$ ), and the labelling of the semi-infinite edges by the limits $x_{0}, \ldots, x_{d} \in L$.

A compactified moduli space for any type is obtained after imposing a stability condition. A holomorphic tree disk $u: C=S \cup T \rightarrow X$ is stable if it has no automorphisms, or equivalently

(a) each disk component $S_{v, \circ} \subset S$ on which the map $u$ is constant (that is, a ghost disk bubble) has at least one interior node $S_{v, \circ} \cap T_{\bullet} \neq \emptyset$ or has at least three boundary nodes $\#\left(S_{v, \circ} \cap T_{\circ}\right) \geq 3$, where $T_{\circ}$ resp. $T_{\bullet}$ denotes the union of segments $e$ corresponding to $e \in$ Edge $_{\circ}$ resp. $e \in$ Edge $_{\bullet}$;

(b) each sphere component $S_{v, \bullet} \subset S$ on which the map $u$ is constant (that is, a ghost sphere bubble) has at least three nodes $\#\left(S_{v, \bullet} \cap T_{\bullet}\right) \geq 3$;

(c) each broken segment $T_{e, i} \subset T_{e}$ on which the map $u$ is constant has at most one infinite end, that is, one of the ends of $T_{e, i}$ is an attaching point to a sphere or disk $S_{v} \subset S$.

Note that the case $C \cong \mathbb{R}$ equipped with a non-constant Morse trajectory $u: C \rightarrow L$ is allowed under this stability condition. The energy of a treed disk is the sum of the energies of the surface components,

$$
E_{H}(u)=\int_{S}(1 / 2)\left|\mathrm{d}_{H} u\right|_{S}^{2} \mathrm{~d} \mathrm{Vol}_{S}
$$

and for holomorphic treed disks with $H=0$ is equal to the symplectic area

$$
A(u)=\int_{S}\left(\left.u\right|_{S}\right)^{*} \omega
$$


For any combinatorial type $\Gamma$ denote by $\mathcal{M}_{\Gamma}(\phi)$ the moduli space of finite energy stable treed holomorphic disks of type $\Gamma$. Denote by

$$
\overline{\mathcal{M}}_{d}(\phi)=\bigcup_{\Gamma} \mathcal{M}_{\Gamma}(\phi)
$$

the union over combinatorial types with $d$ incoming edges.

Proposition 3.2 In a neighborhood of any holomorphic treed disk $u: C \rightarrow X$ of type $\Gamma$ with stable domain the moduli space $\mathcal{M}_{\Gamma}(\phi)$ is cut out by a Fredholm map of Banach spaces.

Proof The proof is standard combination of various Banach spaces of maps and Sobolev multiplication theorems. Let $C=S \cup T$ be the domain of $u$ and denote by $S^{\circ}$ the surface obtained by removing the nodes $q_{k}$ that map to self-intersection points $\phi\left(L \times{ }_{\phi} L-L\right)$. Fix a metric on $S^{\circ}$ with strip-like ends near those self-intersection points. For $k p>2$ let $\operatorname{Map}_{k, p}\left(S^{\circ}, X\right)$ resp. $\operatorname{Map}_{k, p}(T, L)$ denote the space of continuous maps with finite $W^{k, p}$-norm with respect to suitably chosen covariant derivatives on the domains and target. The base of the required bundle is the space

$$
\mathcal{B}_{\Gamma}=\left\{\begin{array}{l}
\left(C, u_{S}, \partial u_{S}, u_{T_{1}}, u_{T_{2}}\right) \in\left(\begin{array}{l}
\mathcal{M}_{\Gamma} \times \operatorname{Map}_{k, p}\left(S^{\circ}, X\right) \times \operatorname{Map}_{k-1 / p, p}\left(\partial S^{\circ}, L\right) \\
\times \operatorname{Map}_{k, p}\left(T_{1}, L\right) \times \operatorname{Map}_{k, p}\left(T_{2}, L \times \phi\right. \\
\end{array}\right) \mid \\
u\left|\partial S^{\circ}=\phi \circ \partial u, \phi \circ u_{T_{1}}\right| S^{\circ} \cap T_{1}=u_{S} \mid \partial S^{\circ} \cap T_{1}
\end{array}\right\} .
$$

Note that by the Sobolev trace theorem the boundary map $\operatorname{Map}_{k, p}\left(S^{\circ}, X\right)$ takes values in $\operatorname{Map}_{k-1 / p, p}\left(\partial S^{\circ}, X\right)$, so the boundary condition $u \mid \partial S^{\circ}=\phi \circ \partial u$ is well-defined. Local charts for $\mathcal{B}_{\Gamma}$ can be constructed using geodesic exponentiation exp :TX $\rightarrow X$ for some metric for which $L$ is totally geodesic; such a metric exists as long as the self-intersections of $L$ are self-transverse. Given such a metric we have compatible maps

$$
\Omega\left(S^{\circ}, u^{*} T X\right)_{k, p} \rightarrow \operatorname{Map}_{k, p}\left(S^{\circ}, X\right) \quad \Omega\left(\partial S^{\circ}, u^{*} T L\right)_{k-1 / p, p} \rightarrow \operatorname{Map}_{k-1 / p, p}\left(\partial S^{\circ}, L\right)
$$

providing the local charts for $\mathcal{B}_{\Gamma}$. The fiber of the bundle $\mathcal{E}_{\Gamma}$ over some map $u$ is the vector space

$$
\mathcal{E}_{\Gamma, u}:=\Omega^{0,1}\left(S, u_{S}^{*} T X\right)_{k-1, p} \oplus \Omega^{1}\left(T_{1}, u_{T_{1}}^{*} T L\right)_{k-1, p} \oplus \Omega^{1}\left(T_{2}, u_{T_{2}}^{*} T L^{2}\right)_{k-1, p} .
$$

Local charts are provided by almost complex parallel transport

$$
\Pi_{u}^{\xi}: \Omega^{0,1}\left(S, \exp _{u}(\xi)_{S}^{*} T X\right)_{k-1, p} \rightarrow \Omega^{0,1}\left(S, u_{S}^{*} T X\right)_{k-1, p}
$$


along $\exp _{u}(s \xi)$ for $s \in[0,1]$. However, in general $\mathcal{E}_{\Gamma} \rightarrow \mathcal{B}_{\Gamma}$ is only a $C^{0}$-Banach manifold, because the transition maps between the local trivializations involve reparametrizing the domains $S$ in $C=S \cup T$ and these reparametrization actions are not smooth on Sobolev spaces $W^{k, p}(S)$.

However, in any local trivialization of the universal curve one can obtain Banach bundles with arbitrarily high regularity. Let $\mathcal{U}_{\Gamma}^{i} \rightarrow \mathcal{M}_{\Gamma}^{i} \times C$ be a collection of local trivializations of the universal curve. Let $\mathcal{B}_{\Gamma}^{i}$ denote the inverse image of $\mathcal{M}_{\Gamma}^{i}$ in $\mathcal{B}_{\Gamma}$ and $\mathcal{E}_{\Gamma}^{i}$ its preimage in of $\mathcal{E}_{\Gamma}$. For integers $k, p$ determining the Sobolev class as above the Fredholm map cutting out the moduli space over $\mathcal{M}_{\Gamma}^{i}$ is

$$
\mathcal{F}_{\Gamma}^{i}: \mathcal{B}_{\Gamma}^{i} \rightarrow \mathcal{E}_{\Gamma}^{i}, u \mapsto\left(\bar{\partial}_{u \mid S}, \frac{d}{d s} u_{T_{1}}+\operatorname{grad}\left(m\left(u_{T_{1}}\right)\right), \frac{d}{d s} u_{T_{2}}\right) .
$$

In other words, $u$ is $J$-holomorphic on the surface parts $S \subset C$, a gradient trajectory of $-m$ on the unbranched segments $T_{e} \subset T^{1}$ and constant on the branched segments $T_{e} \subset T^{2}$. Indeed, in local charts and after restricting to a sheet of the immersion $\iota: L \rightarrow X$, the condition $u \mid \partial S^{\circ}=\phi \circ \partial u$ simply imposes Lagrangian boundary conditions. After imposing a finite energy condition each strip-like end limiting to such a node has a well-defined limit $u\left(q_{j, \pm}\right) \in L \times{ }_{\phi} L$. The zero set of $\mathcal{F}_{\Gamma}^{i}$ need not satisfy the matching conditions $u\left(q_{j,-}\right)=u\left(q_{j,+}\right)$ on the nodes of $S$ mapping to self-intersection points. The matching condition may be imposed a posteriori, that is, $\mathcal{M}_{\Gamma}(\phi)$ is the subset of $\mathcal{F}_{\Gamma}^{-1}(0)$ such that for each node $u\left(q_{j,-}\right)=u\left(q_{j,+}\right)$. Since the set of self-intersection points $L \times_{\phi} L-\Delta_{L}$ is finite, this identifies $\mathcal{M}_{\Gamma}(\phi)$ as a connected component of the zero set $\mathcal{F}_{\Gamma}^{-1}(0)$. Elliptic regularity for pseudoholomorphic curves in [43, Theorem B.4.1] (note that the boundary condition is embedded locally) imply that the solution space consists of smooth maps.

The linearization of the map (23) cutting out the moduli space is a combination of the standard linearization of the Cauchy-Riemann operator with additional terms arising from the gradient operator and variation of conformal structure. With $k, p$ integers determining the Sobolev class as above let

$$
\begin{aligned}
D_{u}: \Omega^{0}\left(S, u_{S}^{*} T X,\left(\partial u_{S}\right)^{*} T L\right)_{k, p} & \rightarrow \Omega^{0,1}\left(S, u_{S}^{*} T X\right)_{k-1, p} \\
\xi & \mapsto \nabla_{H}^{0,1} \xi-\frac{1}{2}\left(\nabla_{\xi} J\right) J \partial_{H} u_{S}
\end{aligned}
$$

denote the linearization of the Cauchy-Riemann operator, c.f. McDuff-Salamon [43, p. 258]; here $\partial_{H} u_{S}=(1 / 2)\left(J \mathrm{~d}_{H} u_{S}+\mathrm{d}_{H} u_{S j}\right)$ and $\nabla_{H}^{0,1} \xi$ is the 0,1 -projection of $\nabla_{H} \xi=\nabla \xi-\nabla_{\xi} H$, where $H \in \Omega^{1}\left(S, \operatorname{Vect}_{h}(X)\right)$ is the Hamiltonian vector field. Denote by $\tilde{D}_{u}$ the operator given by combining the linearization of the Cauchy-Riemann 
operator with the linearized gradient operator and the variation of conformal structure and metrics on the domain:

$$
\begin{aligned}
& \tilde{D}_{u}: T_{[C]} \mathcal{M}_{\Gamma} \oplus \Omega^{0}\left(S^{\circ}, u_{S}^{*} T X\right)_{k, p} \oplus \Omega^{0}\left(T_{1}, u_{1}^{*} T L\right)_{k, p} \oplus \Omega^{0}\left(T_{2}, u_{2}^{*} T L^{2}\right)_{k, p} \\
& \rightarrow \Omega^{0,1}\left(S, u^{*} T X\right)_{k-1, p} \oplus \Omega^{1}\left(T_{1}, u^{*} T L\right)_{k-1, p} \oplus \Omega^{1}\left(T_{2}, u^{*} T L^{2}\right)_{k-1, p} \oplus \operatorname{Map}\left(S \cap T, u^{*} T X\right), \\
& \left(\zeta, \xi_{S}, \xi_{1}, \xi_{2}\right) \mapsto\left(\begin{array}{l}
D_{u} \xi_{S}-(1 / 2) J \mathrm{~d} u \zeta, \nabla \xi_{1}+D_{\xi} \operatorname{grad}(m(u)), \nabla \xi_{2}, \\
\left.\xi_{S}\right|_{S \cap T}-\left(\left.\left.D \phi \xi_{1}\right|_{T_{1} \cap S} \sqcup D \phi \xi_{2}\right|_{T_{2} \cap S}\right)
\end{array}\right) .
\end{aligned}
$$

Here the last factor $\operatorname{Map}\left(S \cap\left(T_{1}, T_{2}\right), u^{*} T X\right)$ enforces the matching condition for the sections $\xi_{S}, \xi_{1}, \xi_{2}$ at the finite set of nodes $S \cap\left(T_{1} \cup T_{2}\right) \subset C$. A holomorphic treed disk $u: C \rightarrow X$ with stable domain $C$ is regular if the linearized operator $\tilde{D}_{u}$ is surjective, and rigid if it is regular and $\tilde{D}_{u}$ is an isomorphism, or more generally, if $C$ is unstable, if $\tilde{D}_{u}$ is surjective and the kernel of $\tilde{D}_{u}$ is generated by the infinitesimal automorphism $\operatorname{aut}(C)$ of $C$.

A standard application of the implicit function theorem in Banach spaces implies that the moduli space of rigid regular disks is invariant under small perturbations of the boundary condition or almost complex structure:

Corollary 3.3 Let $u_{0}: C \rightarrow X$ be a rigid regular holomorphic treed disk with boundary in a Lagrangian immersion $\phi_{0}: L \rightarrow X$ with respect to a symplectic form $\omega_{0}$. Suppose that $\phi_{t}: L \rightarrow X$ is a family of Lagrangian immersions with respect to a family of symplectic forms $\omega_{t}$ for $t \in[-T, T]$, agreeing with the given immersion and form at $t=0$. Let $D \subset X$ a Donaldson hypersurface symplectic for all $\omega_{t}$ and $P_{\Gamma, t}$ a family of perturbation data for adapted holomorphic treed disks for combinatorial type $\Gamma_{0}$. Then

(a) (Existence) there exists an $\epsilon>0$ such that there exists a family $u_{t}: C \rightarrow X$ for $|t|<\epsilon$ of holomorphic treed disks with boundary in the Lagrangian immersion $\phi_{t}: L \rightarrow X$;

(b) (Uniqueness) the solution $u_{t}$ is unique in the following sense: There exists a $\delta>0$ such that $u_{t}$ is the unique such map up to isomorphism in an open neighborhood of $u_{0}$ in the Gromov topology.

(c) (Uniformity) The sizes of $\delta$ and $\epsilon$ depend on uniform estimates for the distance from $\phi_{0}$ to $\phi_{t}$ and from $P_{\Gamma, 0}$ to $P_{\Gamma, 1}$ in $C^{1}$ norm and a lower bound for the right inverse of the linearized operator $\tilde{D}_{u}$.

The moduli space of holomorphic treed disks admits a natural version of the Gromov topology which allows bubbling off spheres, disks, and Morse trajectories. Given 
a sequence $u_{\nu}: C_{\nu}=S_{\nu} \cup T_{\nu} \rightarrow X, \partial u_{\nu}: \partial C_{\nu} \rightarrow L$ of treed holomorphic disks with boundary in $\phi$ with bounded energy Gromov compactness of pseudoholomorphic curves with Lagrangian boundary conditions as in, for example, Frauenfelder-Zemisch [30] implies that there exist stable limits $\lim u_{\nu} \mid S_{\nu} \rightarrow X$ on the surface parts, while limits on the tree parts $\lim \partial u_{\nu} \mid \partial C_{\nu} \rightarrow L$ are a consequence of convergence for gradient trajectories up to breaking. Thus for any fixed energy bound $E$, the subset

$$
\overline{\mathcal{M}}_{d}^{<E}(\phi)=\left\{u \in \overline{\mathcal{M}}_{d}(\phi) \mid E(u)<E\right\}
$$

satisfying the given energy bound is compact.

The moduli space further decomposes according to the limits at infinity and the expected dimension. For each end $e=0, \ldots, d$ let $\epsilon_{e}:[0, \infty) \rightarrow C$ denote a local coordinate on the $e$-th end. Define the sets of possible limits

$$
\begin{aligned}
& \mathcal{I}^{\mathrm{c}}(\phi)=\operatorname{crit}(m)=\{x \in L \mid \mathrm{d} m(x)=0\} \\
& \mathcal{I}^{\mathrm{si}}(\phi)=\left\{\left(x_{-}, x_{+}\right) \in L^{2} \mid \phi\left(x_{-}\right)=\phi\left(x_{+}\right), x_{-} \neq x_{+}\right\} .
\end{aligned}
$$

The set of self-intersections $\mathcal{I}^{\text {si }}(\phi)$ has a natural involution

$$
\mathcal{I}^{\mathrm{si}}(\phi) \rightarrow \mathcal{I}^{\mathrm{si}}(\phi), x=\left(x_{-}, x_{+}\right) \mapsto \bar{x}=\left(x_{+}, x_{-}\right) .
$$

Given

$$
\underline{x}=\left(x_{0}, \ldots, x_{d}\right) \in \mathcal{I}^{\mathrm{c}}(\phi) \cup \mathcal{I}^{\mathrm{si}}(\phi)
$$

denote by

$$
\overline{\mathcal{M}}_{d}(\phi, \underline{x})=\left\{[u: C \rightarrow X] \in \overline{\mathcal{M}}_{d}(\phi, \underline{x}) \mid \lim _{s \rightarrow \infty} u\left(\epsilon_{e}(s)\right)=x_{e}, \forall e=0, \ldots, d\right\}
$$

the locus with limits $\underline{x}$. For any integer $p$ denote by

$$
\overline{\mathcal{M}}_{d}(\phi)_{p}=\left\{[u: C \rightarrow X] \mid \operatorname{Ind}\left(\tilde{D}_{u}\right)-\operatorname{dim}(\operatorname{aut}(C))=p\right\}
$$

the locus with expected dimension $p$, where $\tilde{D}_{u}$ is the operator of (25). In the next section, we construct perturbed moduli spaces using Cieliebak-Mohnke perturbations [15] and show that they are still compact.

\section{Transversality and compactness}

In this section we construct perturbations of the moduli spaces of treed holomorphic disks with immersed Lagrangian boundary conditions so that the perturbed moduli spaces are transversally cut out and compact. To achieve transversality, we combine 
the approach in Akaho-Joyce [2] with Biran-Cornea [8], Charest [12], and CharestWoodward [13] which uses Donaldson hypersurfaces to stabilize the domains of the holomorphic treed disks; once the domains are stabilized, one may choose generic domain-dependent almost complex structures and Morse functions to achieve regularity, except for the following trivial case: To achieve transversality for constant maps to self-intersections of the Lagrangian immersion domain-dependent Hamiltonian perturbations are required.

The existence of suitable Donaldson hypersurfaces uses auxiliary line bundles whose curvature is the symplectic form, and so requires rationality assumptions. Say that $\phi$ is weakly rational if the pairing of $[\omega] \in H^{2}(\phi)$ with any relative cycle is rational. That is, the class $[\omega]$ lies in the image of the natural map from singular cohomology with rational coefficients:

$$
[0, \omega] \in \operatorname{Im}\left(H^{2}(\phi, \mathbb{Q}) \rightarrow H^{2}(\phi, \mathbb{R})\right) .
$$

In particular, $[\omega] \in H^{2}(X, \mathbb{Q})$ so that for some integer $k, k[\omega]=c_{1}(\tilde{X})$ for some line bundle $\tilde{X} \rightarrow X$. By Stokes' theorem the pairings of $[\omega]$ with disk classes $u_{*}[S] \in H_{2}(\phi)$ are related to the holonomies of the connection around the boundary by

$$
\exp \left(2 \pi i\left(u_{*}[S], k[\omega]\right)\right)=\operatorname{Hol}\left((\partial u)^{*} \tilde{X}\right)
$$

and so trivial. The immersion $\phi$ is strongly rational if there exists a line bundle $\tilde{X} \rightarrow X$ with connection whose curvature equals $k(2 \pi / i) \omega$ for some integer $k>0$ such that the restriction of $\tilde{X}$ to $\phi(L)$ is trivial as a line bundle with connection. That is, there exists an isomorphism of line bundles with connection $\psi: \phi^{*} L \rightarrow L \times \mathbb{C}$ (where $L \times \mathbb{C}$ is equipped with the trivial connection) such that $\phi\left(x_{1}\right)=\phi\left(x_{2}\right)$ implies that $\psi_{x_{1}}$ agrees with $\psi_{x_{2}}$ using the canonical identification $L_{\phi\left(x_{1}\right)}=L_{\phi\left(x_{2}\right)}$.

Strong rationality implies the existence of Donaldson hypersurfaces in the complement of the image of the Lagrangian immersion, similar to the construction in CharestWoodward [13]. Let $\tilde{X} \rightarrow X$ be a line-bundle with connection $\alpha$ over $X$ whose curvature two-form $\operatorname{curv}(\alpha)$ satisfies $\operatorname{curv}(\alpha)=(2 \pi / i) \omega$; since our symplectic manifold $(X, \omega)$ has rational symplectic class $[\omega]$ we may always assume such $\tilde{X}$ after taking a suitable integer multiple of the symplectic form $\omega$. Let $\left(\sigma_{k}\right)_{k \geq 0}$ be a sequence of sections of $\tilde{X}^{k} \rightarrow X$. The sequence $\left(\sigma_{k}\right)_{k \geq 0}$ is asymptotically holomorphic if there exists a constant $C$ and integer $k_{0}$ such that for $k \geq k_{0}$,

$$
\left|\sigma_{k}\right|+\left|\nabla \sigma_{k}\right|+\left|\nabla^{2} \sigma_{k}\right| \leq C, \quad\left|\bar{\partial} \sigma_{k}\right|+\left|\nabla \bar{\partial} \sigma_{k}\right| \leq C k^{-1 / 2} .
$$

The sequence $\left(\sigma_{k}\right)_{k \geq 0}$ is uniformly transverse to 0 if there exists a constant $\eta$ independent of $k$ such that for any $x \in X$ with $\left|\sigma_{k}(x)\right|<\eta$, the derivative of $\sigma_{k}$ is surjective and satisfies $\left|\nabla \sigma_{k}(x)\right| \geq \eta$. In both definitions the norms of the derivatives 
are evaluated using the metric $g_{k}=k \omega(\cdot, J \cdot)$. Donaldson's construction [22] produces from an asymptotically holomorphic sequence of sections a nearby sequence that is uniformly transverse, so that the zero section $D=\sigma_{k}^{-1}(0)$ is a symplectic submanifold of codimension two representing $k[\omega]$, called a Donaldson hypersurface.

Lemma 4.1 Suppose that $\phi: L \rightarrow X$ is a strongly rational Lagrangian immersion with only transverse self-intersections or non-degenerate tangencies. There exists a Donaldson hypersurface $D \subset X$ with the property that $\phi(L)$ is exact in $X-D$. That is, there exists a one-form

$$
\alpha \in \Omega^{1}(X-D), \quad d \alpha=\omega
$$

and a function

$$
\beta: L \rightarrow \mathbb{R}, \quad \mathrm{d} \beta=\phi^{*} \alpha
$$

such that for any $x_{1}, x_{2} \in L$, equality $\phi\left(x_{1}\right)=\phi\left(x_{2}\right)$ implies $\beta\left(x_{1}\right)=\beta\left(x_{2}\right)$. In particular, any disk with boundary in $L$ with non-zero area intersects $D$.

Proof The first step is to choose an asymptotically holomorphic sequence of sections concentrated on the image of the immersion. By the Auroux-Gayet-Mohsen [6] prescription, there exists a Gaussian asymptotically holomorphic sequence of sections $\sigma_{k}: X \rightarrow \tilde{X}^{k}$ concentrated on $\phi(L)$. At least locally we may model $X$ in a neighborhood $U$ of the image of $L$ as the cotangent bundle $T^{\vee} L$ with coordinates $q_{1}, \ldots, q_{n}, p_{1}, \ldots, p_{n}$. Parallel transport along geodesics $\exp _{x}(s v), v \in T_{x} X$ normal to $L$ defines a local trivialization of $\tilde{X}$ near the image of $\phi(L)$, depending on the choice of branch. An asymptotically-holomorphic sequence of sections $\sigma_{k, \text { loc }}: U \rightarrow \tilde{X} \mid U$ is then given by

$$
\sigma_{k, \mathrm{loc}}\left(q_{1}, \ldots, q_{n}, p_{1}, \ldots, p_{n}\right)=\exp \left(-k\left(p_{1}^{2}+\ldots+p_{n}^{2}\right)\right) .
$$

The sequence $\sigma_{k \text {,loc }}$ can be extended to a globally defined sequence of sections $\sigma_{k}$ on $X$ by multiplying by a cutoff function $\varkappa: X \rightarrow \mathbb{R}$ supported in $U$ and equal to 1 in neighborhood of each branch of $\phi(L)$. Near a transverse self-intersection or admissible self-tangent intersection $x \in L \times_{\phi} L-\Delta_{L}$, the section $\sigma_{k}$ is the sum of the contributions $\sigma_{k, 1}+\sigma_{k, 2}$ from the branches. The sum $\sigma_{k}$ is non-vanishing on each branch of $\phi(L)$, since the maximum of the norm of $\sigma_{k, m}$ occurs on the branch $\phi(L)_{m}$. Since $\sigma_{k, m}, m \in\{1,2\}$ restricts to a section on $L_{m+1}$ (with $m \bmod 2$ ) of norm strictly less than one, the sum $\left(\sigma_{m}+\sigma_{m+1}\right) \mid L_{m}$ is homotopic to $\sigma_{m} \mid L_{m}$, and so the Maslov computation still implies that the intersection number with $\sigma^{-1}(0)$ is the symplectic area. 
In the second step one applies Donaldson's perturbation procedure [22] to obtain asymptotically holomorphic sections $\sigma_{k}^{\prime}: X \rightarrow \tilde{X}^{k}$ uniformly transverse to the zero section $X \subset \tilde{X}^{k}$. This gives a symplectic hypersurface $D_{k}:=\left(\sigma_{k}^{\prime}\right)^{-1}(0)$ in the complement of the Lagrangian. Because the section $\sigma_{k}^{\prime}$ is homotopic to a covariant constant section of $\tilde{X}^{k}$ on the Lagrangian $L$, on the complement of $D_{k}$ we have

$$
k \omega=\mathrm{d} \alpha_{k}, \alpha_{k} \mid L=\mathrm{d} f_{k}
$$

where $\alpha_{k}$ is the connection one-form corresponding to the section $\sigma_{k}$. Thus the Lagrangian $L$ is exact in the complement $X-D_{k}$ of the Donaldson hypersurface $D_{k} \subset X$.

Given a weakly rational immersion one may choose a small perturbation of the symplectic form and connection to obtain a strongly rational immersion with respect to a slightly different symplectic form:

Lemma 4.2 Let $\phi: L \rightarrow X$ be a weakly rational immersion with only isolated self-intersections $\phi\left(x_{k,+}\right)=\phi\left(x_{k,-}\right)$ for $k=1, \ldots, m$. There exists a collection of paths

$$
\left\{\gamma_{k, l, \pm, \pm}:[0,1] \rightarrow L \mid(k \pm, l \pm) \in \Theta\right\}
$$

where $\gamma_{k, l, \pm, \pm}$ is a path from $x_{k, \pm}$ to $x_{l, \pm}$ in $L$ that are disjoint except for the endpoints and $\Theta \subset \mathcal{I}^{\text {si }}(\phi)$ is an indexing set such that all distinct pairs of self-intersection points are connected by a concatenation of such paths. For a generic perturbation of the connection one-form $\alpha_{t}^{\prime}$ that is closed on $L$, the parallel transports along $\gamma_{k, l, \pm, \pm}$ are rational and so $\phi: L \rightarrow X$ is strongly rational with respect to the resulting symplectic form $\omega^{\prime}$.

Proof The strategy of proof is to introduce a variation of connection and symplectic form on each branch individually, which preserves the Lagrangian condition. The proof is slightly different in the case of surface target. If $\operatorname{dim}(X)>2$ then $\Theta$ can be chosen to connect all self-intersection points, and if $\operatorname{dim}(X)=2$ then $\Theta$ can be chosen to connect adjacent self-intersection points. By assumption the self-intersections $\phi\left(x_{k, \pm}\right)$ are isolated, so that at any self-intersection point $\phi\left(x_{k, \pm}\right)$ there are two branches $L_{0}, L_{1}$ of $\phi(L)$ in any sufficiently small neighborhood of a self-intersection point $\phi\left(x_{k, \pm}\right)$.

To produce the variation in connection, let $f: \mathbb{R}_{\geq 0} \rightarrow[0,1]$ be a smooth bump function supported in $[0,2]$ and equal to 1 on $[0,1]$. Let $x \in L$ be a point on which $\phi$ is not injective, that is, $\phi(x)=\phi(y)$ for some $y \neq x \in L$. Choose local Darboux coordinates $p_{1}, \ldots, p_{n}, q_{1}, \ldots, q_{n}$ on $X$ centered at $\phi(x)$ so that the given branch of $\phi(L)$ is equal 


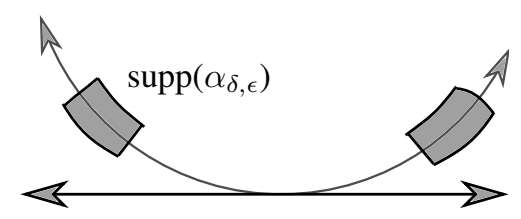

Figure 7: Support of the perturbation of the connection one-form

near $x$ to the locus defined by $p_{1}=\ldots=p_{n}=0$. Consider perturbations $\alpha^{\prime}$ of the connection $\alpha$ by multiples of the one-forms

$$
\alpha_{\delta, \epsilon}=f(\epsilon|p|) \mathrm{d} f(\delta|q|) ;
$$

the support is shown in Figure 7. In the Figure, the branch $\phi(L)$ on which the connection is modified is the top branch. Since the non-injective points are assumed isolated, for sufficiently small $\delta, \epsilon$ the support of $\alpha_{\delta, \epsilon}$ does not intersect any other branch of $\phi(L)$. The effect of adding $\alpha_{\delta, \epsilon}$ to the connection one-form on $\tilde{X}$ is to modify the parallel transport in the fibers of $\tilde{X}$ along any path $\gamma$ to or from $x$ by a small amount $\delta h \in S^{1}$, without changing the parallel transports to $x$ along other branches of $\phi(L)$, at the cost of a small change $\delta \omega$ in the symplectic form $\omega$ which keeps the immersion $\phi$ Lagrangian, that is, $\phi^{*}(\omega+\delta \omega)=0$. Thus we ensure that there is a path between each pair of self-intersection points which has rational holonomy. Since this perturbation preserves the holonomy of each loop we still have that each $\gamma: S^{1} \rightarrow L$ has rational holonomy for $\phi^{*} \tilde{X}$, all paths $\gamma_{k, l, \pm, \pm}$ from (27) between self-intersection points $x_{k}, x_{l}$ have the property that the loops $\phi\left(\gamma_{k, \pm} \gamma_{l, \pm}^{-1}\right)$ have rational holonomy. After taking a suitable tensor power $\tilde{X}^{\otimes k}, k \gg 0$ of the bundle $\tilde{X}$, all paths between intersection points have the same parallel transport. It follows that the bundle $\tilde{X}$ admits a trivialization over $\phi(L)$.

Remark 4.3 Given Lemma 4.2, we may assume that the Lagrangian immersions $\phi_{s}: L \rightarrow X$ for rational times $s$ are strongly rational. Indeed, suppose that $L$ is exact in $X-D$ with respect to some perturbed symplectic form $\omega^{\prime}$. The number of intersection points $u^{-1}(D)$ of $u$ with $D$ is $k$ times the pairing of $u_{*}[C]$ with $\left[\omega^{\prime}\right]$; the number $\# u^{-1}(D)$ is necessarily positive for any almost complex structure $J \in \mathcal{J}_{\tau}(X, \omega) \cap J_{\tau}\left(X, \omega^{\prime}\right)$ taming both symplectic forms $\omega, \omega^{\prime}$ which exists as long as $\omega^{\prime}$ is sufficiently close to $\omega$.

Domain-dependent perturbations as in Cieliebak-Mohnke [15] are chosen using the Donaldson hypersurfaces above. For sufficiently large degree and a generic almost complex structure $J_{D} \in \mathcal{J}_{\tau}(X)$ making $D$ an almost complex submanifold, the Donaldson hypersurface $D$ contains no pseudoholomorphic spheres $v: \mathbb{P}^{1} \rightarrow D$ and any 
pseudoholomorphic sphere $v: \mathbb{P}^{1} \rightarrow X$ intersects the hypersurface $D \subset X$ in at least three but finitely many points $v^{-1}(D)$ [15, Proposition 8.11]. The additional intersection points of a holomorphic treed disk $u: C \rightarrow X$ with a Donaldson hypersurface $D$ may be used to stabilize the domains $C$ of holomorphic disks. Then domain-dependent almost complex structures achieve transversality, as we now explain. Call a holomorphic treed disk $u: C=S \cup T \rightarrow X$ adapted to the Donaldson hypersurface $D \subset X$ if each bulk leaf $T_{e}$ (with terminology from Section 3) lies in $u^{-1}(D)$ and each connected component of $u^{-1}(D)$ contains an bulk leaf $T_{e}, e \in$ Edge $_{\bullet} \rightarrow(\Gamma)$; in the absence of sphere bubbles $S_{v}, v \in \operatorname{Vert}_{\text {o }}(\Gamma)$ mapping to the hypersurface $D$ this means that the bulk leaves are the intersection points with $D$, that is,

$$
u^{-1}(D)=\bigcup_{e \in \mathrm{Edge}_{\bullet} \rightarrow(\Gamma)} T_{e} .
$$

Let

$$
\mathcal{J}_{\tau}(X)=\left\{J: T X \rightarrow T X \mid J^{2}=-\mathrm{Id}, \quad \omega(\cdot, J \cdot)>0\right\}
$$

denote the space of tame almost complex structures on $X$. As in Cieliebak-Mohnke [15, Section 8], there exist an open subset $\mathcal{J}_{\tau}\left(X, J_{D}, \Gamma\right)$ in the space of such domaindependent almost complex structures $J$ near $J_{D}$ with the property that each $J$ holomorphic sphere $u \mid S_{v}: S_{v} \rightarrow X, v \in \operatorname{Vert}_{\bullet}(\Gamma)$ intersects $D$ in finitely many but at least three points. For each combinatorial type $\Gamma$, a domain-dependent almost complex structure is a map

$$
J_{\Gamma}: \overline{\mathcal{S}}_{\Gamma} \rightarrow \mathcal{J}_{\tau}\left(X, J_{D}, \Gamma\right)
$$

(notation from (15)) agreeing with the given almost complex structure $J_{D}$ on the hypersurface $D$ and in a neighborhood of the nodes $q_{k} \in S$ and boundary $S$ for any fiber $S \subset \overline{\mathcal{S}}_{\Gamma}$. We also choose a domain-dependent Hamiltonian perturbation: Let $\operatorname{Vect}_{h}(X) \subset \operatorname{Vect}(X)$ denote the space of Hamiltonian vector fields and $\operatorname{Vect}_{h}(X, D)$ the space of vector fields $v: X \rightarrow T X$ that vanish on an open neighborhood of the Donaldson hypersurface $D \subset X$. A domain-dependent Hamiltonian perturbation is a one-form on $\overline{\mathcal{S}}_{\Gamma}$ with values in $\operatorname{Vect}_{h}(X, D)$

$$
H_{\Gamma} \in \Omega^{1}\left(\overline{\mathcal{S}}_{\Gamma}, \operatorname{Vect}_{h}(X, D)\right)
$$

vanishing on an open neighborhood of the boundary of $\overline{\mathcal{S}}_{\Gamma}$. A domain-dependent Morse function is a map

$$
m_{\Gamma}: \overline{\mathcal{T}}_{\Gamma} \times L \rightarrow \mathbb{R}
$$

agreeing with the given Morse function $m: L \rightarrow \mathbb{R}$ in a neighborhood of the endpoints of each segment $T_{i} \subset T$ for any fiber $T \subset \overline{\mathcal{T}}_{\Gamma}$. A perturbation datum is a triple $P_{\Gamma}=\left(J_{\Gamma}, H_{\Gamma}, m_{\Gamma}\right)$. The space of perturbation data of class $C^{l}$ is denoted $\mathcal{P}_{\Gamma}^{l}=\left\{P_{\Gamma}\right\}$. 
The combinatorial type of an adapted map is that of the map with the additional data of a labelling $d(e), e \in \operatorname{Edge}(\Gamma)$ of any interior node by intersection multiplicity $d(e)$ with the hypersurface $D$; we let $d(e)=0$ if the map is constant with values in the hypersurface $D$ near the node. We denote the moduli space of adapted treed holomorphic disks of type $\Gamma$ by $\mathcal{M}_{\Gamma}(\phi, D)$. Denote by $\overline{\mathcal{M}}(\phi, D)$ the union over combinatorial types. The requirement that the intersections with the Donaldson hypersurface are the bulk leaves means that the energy of a given combinatorial type of map to $X$ is determined by the bulk leaves as well as the set of ghost bubbles. Thus in particular, for any combinatorial type $\Gamma$ of holomorphic treed disk there exist finitely many combinatorial types $\Gamma_{X}$ with domain of type $\Gamma$ (allowing any combination of stable sphere components to be ghost bubbles.) Thus for each type $\Gamma$ of domain the union

$$
\mathcal{M}_{\Gamma}(\phi, D)=\bigcup_{\Gamma \rightarrow \Gamma_{X}} \mathcal{M}_{\Gamma_{X}}(\phi, D)
$$

is a finite union of types $\Gamma_{X}$ of maps.

In order to obtain good compactness properties, we assume the following coherence properties of the perturbations. For each vertex $v \in \operatorname{Vert}(\Gamma)$, let $\Gamma(v)$ denote the subtree of $\Gamma$ consisting of the vertex $v$ and all edges of $\Gamma$ meeting $v$. There is natural inclusion $\pi^{*} \mathcal{U}_{\Gamma(v)} \rightarrow \mathcal{U}_{\Gamma}$ and we assume that the perturbations $\underline{P}=\left(P_{\Gamma}\right)$ satisfy the following axioms:

- (Locality) The next axiom prevents ghost bubbles from forming when intersection points with divisors come together. For each vertex $v \in \operatorname{Vert}(\Gamma)$, let

$$
\Gamma(v)=\cup_{e \ni v} e
$$

denote the subtree of $\Gamma$ consisting of the vertex $v$ and all edges $e$ of $\Gamma$ meeting $v$. Let $\Gamma_{\circ}$ denote the subgraph of $\Gamma$ whose vertices are $v \in \operatorname{Vert}_{\circ}(\Gamma)$. Let

$$
\pi=\pi_{1} \times \pi_{2}: \mathcal{U}_{\Gamma} \rightarrow \mathcal{M}_{\Gamma_{\circ}} \times \mathcal{U}_{\Gamma(v)}
$$

be the product of the maps where $\pi_{1}$ is given by projection followed by forgetful morphism and $\pi_{2}$ is the map $C \mapsto S_{v}$, equipped with its special points. The perturbations $P_{\Gamma}$ are local for $v$ if and only if $P_{\Gamma}$ restricts on $S_{v}$ to the pullback under $\pi$ of a family of perturbation data $P_{\Gamma, v}$ on $\mathcal{M}_{\Gamma_{\circ}} \times \mathcal{U}_{\Gamma(v)}$ to $\mathcal{U}_{\Gamma}$. (The dependence on $\mathcal{M}_{\Gamma_{0}}$ is necessary to allow compatibility with the (Cutting Edges) axiom; we thank Guangbo Xu for pointing this out.)

(Cutting-edges axiom) If $\Gamma$ is obtained from types $\Gamma_{1}, \Gamma_{2}$ by gluing along semiinfinite edges then $P_{\Gamma}$ is the product of the perturbations $P_{\Gamma_{1}}, P_{\Gamma_{2}}$ under the isomorphism $\mathcal{U}_{\Gamma} \cong \pi_{1}^{*} \mathcal{U}_{\Gamma 1} \cup \pi_{2}^{*} \mathcal{U}_{\Gamma_{2}}$. 
(Collapsing-edges axiom) If $\Gamma^{\prime}$ is obtained from $\Gamma$ by setting a length equal to zero or infinity or collapsing an edge then the restriction of $P_{\Gamma}$ to $\mathcal{U}_{\Gamma} \mid \mathcal{M}_{\Gamma^{\prime}} \cong \mathcal{U}_{\Gamma^{\prime}}$ is equal to $P_{\Gamma^{\prime}}$.

The origins of the axioms is rather different: the (Cutting-edges) and (Collapsingedges) axioms in particular imply that the moduli space $\mathcal{M}_{\Gamma}(\phi, D)$ over the image of the inclusion $\mathcal{M}_{\Gamma_{1}} \times \mathcal{M}_{\Gamma_{2}} \rightarrow \overline{\mathcal{M}}_{\Gamma}$ is a product of moduli spaces over $\mathcal{M}_{\Gamma_{1}}(\phi, D)$ and $\mathcal{M}_{\Gamma_{2}}(\phi, D)$; this implies that the terms in the $A_{\infty}$ axiom are associated to the boundary points on the moduli space of holomorphic tree disks. On the other hand, in principle one could also have sphere bubbling: Cieliebak-Mohnke perturbations $\underline{P}=\left(P_{\Gamma}\right)$ [15] do not make all strata $\overline{\mathcal{M}}_{\Gamma}(\phi, D)$ expected dimension in the case of ghost bubbles. Without the (Locality axiom), this fact could cause additional terms in the boundary of the one-dimensional component $\overline{\mathcal{M}}(\phi, D)_{1}$ of the moduli space of treed holomorphic maps $\overline{\mathcal{M}}(\phi, D)$. The (Locality axiom) implies that if at least one ghost sphere bubble $u: S_{v} \rightarrow X, u_{*}\left[S_{v}\right]=0$ appears then forgetting all but one marking $z_{k} \in S_{v}$ on each ghost bubble $S_{v} \subset S, d(v)=0$, one obtains a configuration of lower expected dimension $u^{\prime}: S \rightarrow X, \underline{z}^{\prime} \subset \underline{z}$ at least two lower. However, there is no recursive constraint on such perturbations $P_{\Gamma, v}$.

Obtaining strict units requires the addition of weightings to the combinatorial types as in Ganatra [33] and Charest-Woodward [16]. A weighting for a type $\Gamma$ is a map $\varkappa$ from the space $\operatorname{Edge}_{\infty}(\Gamma)$ of semi-infinite edges of $\Gamma$ to $[0, \infty]$. The set of generators of the space of Floer cochains $C F(\phi)$ is enlarged by adding two new elements $x^{\nabla}, x^{\nabla}$ of degree 0 resp. -1 , with the constraint that if the weight $\varkappa(e)$ is non-zero then the only allowable labels of the edge $e$ are $x$ (if the weight is infinite) $x^{\nabla}$ (for any weighting). One then requires that the perturbation system satisfies a forgetful axiom:

(Forgetful axiom) For any semi-infinite edge $e$ with infinite weighting $\varkappa(e)=$ $\infty$, the perturbation datum $P_{\Gamma}$ is pulled back from the perturbation datum $P_{\Gamma^{\prime}}$ under the forgetful map $\mathcal{U}_{\Gamma} \rightarrow \mathcal{U}_{\Gamma^{\prime}}$ obtained by forgetting that semi-infinite edge and stabilizing.

In particular, this axiom implies that the resulting moduli spaces admit forgetful morphisms $\mathcal{M}_{\Gamma}(\phi, D) \rightarrow \mathcal{M}_{\Gamma^{\prime}}(\phi, D)$ whenever there is a semi-infinite edge with infinite weighting. See [16] for more details on the allowable weightings.

In order to apply the Sard-Smale theorem we construct a suitable Banach manifold of perturbation data for each type. We assume perturbation data $P_{\Gamma}$ matches that obtained by gluing perturbation data $P_{\Gamma^{\prime}}$ on strata $\mathcal{U}_{\Gamma^{\prime}}$ contained in $\overline{\mathcal{U}}_{\Gamma}$ on a fixed neighborhood of the boundary. The space $\mathcal{P}_{\Gamma}^{l}$ of perturbation data of class $C^{l}$ taking values in $\mathcal{J}_{\Gamma}\left(J_{D}\right)$ is a Banach manifold and the space $\mathcal{P}_{\Gamma}$ of perturbation data of class $C^{\infty}$ and fixed to 
be the given almost complex structure $J_{D}$ and Morse function $m$ on the complement of a compact subset is a Baire space, by the usual combination of $C^{k}$ norms into a non-linear metric:

$$
\operatorname{dist}\left(P_{\Gamma, 1}, P_{\Gamma_{2}}\right)=\sum_{k \geq 0} 2^{-k} \min \left(1,\left\|P_{\Gamma, 1}-P_{\Gamma, 2}\right\|_{C^{k}}\right) ;
$$

see for example Royden [49, Chapter 7.8].

Given a relative spin structure for the immersion, orientations on the moduli spaces may be constructed following Fukaya-Oh-Ohta-Ono [31, Orientation chapter], [59]. We may ignore the constraints at the interior nodes $z_{1}, \ldots, z_{m} \in \operatorname{int}(S)$, since the tangent spaces to these markings and the linearized constraints $\mathrm{d} u\left(z_{i}\right) \in T_{u\left(z_{i}\right)} D$ are even dimensional and oriented by the given complex structures. At any regular element $(u: C \rightarrow X) \in \mathcal{M}(\phi, D)$ the tangent space to the moduli space of treed disks is the kernel of the linearized operator

$$
T_{u} \mathcal{M}(\phi, D) \cong \operatorname{ker}\left(\tilde{D}_{u}\right) .
$$

The operator $\tilde{D}_{u}$ is canonically homotopic via family of operators $\tilde{D}_{u}^{t}, t \in[0,1]$ to the operator $0 \oplus D_{u} \oplus \frac{d}{d s}$ on the direct sum in (25). For any vector spaces $V, W$ we have isomorphism $\operatorname{det}(V \oplus W) \cong \operatorname{det}(V) \otimes \operatorname{det}(W)$. The $\operatorname{deformation} \tilde{D}_{u}^{t}, t \in[0,1]$ of operators induces a family of determinant lines $\operatorname{det}\left(\tilde{D}_{u}^{t}\right)$ over the interval $[0,1]$, necessarily trivial, and so (by taking a connection on this family) an identification of determinant lines

$$
\operatorname{det}\left(T_{u} \mathcal{M}(\phi, D)\right) \rightarrow \operatorname{det}\left(T_{C} \mathcal{M}_{\Gamma}\right) \otimes \operatorname{det}\left(D_{u}\right)
$$

well-defined up to isomorphism. (Here $D_{u}$ denotes the linearized operator subject to the constraints that require the attaching points of edges mapping to critical points to map to the corresponding unstable manifolds of the Morse function.) In the case of nodes of $S$ mapping to self-intersection points $x \in \mathcal{I}^{\text {si }}(\phi)$ the $\operatorname{determinant} \operatorname{line} \operatorname{det}\left(D_{u}\right)$ is oriented by "bubbling off one-pointed disks", see [31, Theorem 44.1] or [59, Equation (36)]. That is, for each self-intersection point $\left(x_{-} \neq x_{+}\right) \in L^{2}, \phi\left(x_{-}\right)=\phi\left(x_{+}\right)$choose a path of Lagrangian subspaces

$$
\gamma_{x}:[0,1] \rightarrow \operatorname{Lag}\left(T_{\phi\left(x_{-}\right)=\phi\left(x_{+}\right)} X\right), \quad \gamma_{x}(0)=D_{x_{-}} \phi\left(T_{x_{-}} L\right) \quad \gamma_{x}(1)=D_{x_{+}} \phi\left(T_{x_{+}} L\right) .
$$

Let $S$ be the unit disk with a single boundary marking $1 \in \partial S$. The path $\gamma_{x}$ defines a totally real boundary condition on $S$ on the trivial bundle with fiber $T_{x} X$. Let $\operatorname{det}\left(D_{x}\right)$ denote the determinant line for the Cauchy-Riemann operator $D_{x}$ with boundary conditions $\gamma_{x}$ as in [59]. Denote by

$$
i(x)=\operatorname{dim}\left(\operatorname{ker}\left(D_{x}\right)\right)-\operatorname{dim}\left(\operatorname{coker}\left(D_{x}\right)\right) \in \mathbb{Z}
$$




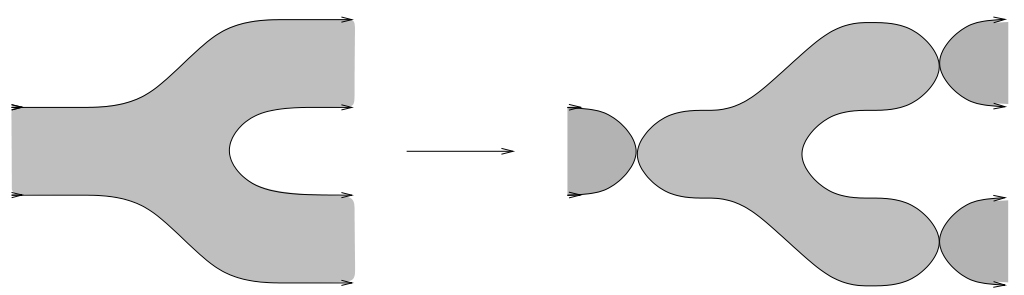

Figure 8: Bubbling off the strip-like ends

the index of the operator $D_{x}$. Let $\mathbb{D}_{x_{k}, 1}^{+}=\operatorname{det}\left(D_{x_{k}, 1}^{+}\right)$and let $\mathbb{D}_{x_{k}, 1}^{-}$be the tensor product of the determinant line $\operatorname{det}\left(D_{x_{k}}^{-}\right)$for the once-marked disk with $\operatorname{det}\left(T_{x_{k}} L\right)$. Because the once-marked disks with boundary conditions $\gamma_{x_{k}}$ and $\gamma_{\overline{x_{k}}}$ glue together to a trivial problem on the disk with index $T_{x_{k}} L$, there is a canonical isomorphism

$$
\mathbb{D}_{x_{k}, 1}^{-} \otimes \mathbb{D}_{x_{k}, 1}^{+} \rightarrow \mathbb{R}
$$

A choice of orientations $O_{x_{k}} \in \mathbb{D}_{x_{k}, 1}^{ \pm}$for the self-intersection points $x_{k}$ are coherent if the isomorphisms (30) are orientation preserving with respect to the standard orientation on $\mathbb{R}$. Similarly for each critical point $x \in \operatorname{crit}(m)$ let $W_{x}^{ \pm} \subset L$ denote the stable and unstable manifolds of $x$ under the flow of $-\operatorname{grad}(m) \in \operatorname{Vect}(L)$. Choices of orientations $O_{x}$ on the determinant lines of the stable resp. unstable manifolds $\operatorname{det}\left(T W_{x}^{ \pm}\right)$are coherent if the natural maps

$$
\operatorname{det}\left(T W_{x}^{-}\right) \otimes \operatorname{det}\left(T W_{x}^{-}\right) \rightarrow \operatorname{det}\left(T_{x} L\right)
$$

are orientation preserving. Set

$$
\mathbb{D}_{x_{k}, 2}^{+}=\operatorname{det}\left(T_{x_{k}} W^{+}\left(x_{k}\right)\right), \quad \mathbb{D}_{x_{k}, 2}^{-}=\operatorname{det}\left(T_{x_{k}} W^{-}\left(x_{k}\right)\right) \otimes \operatorname{det}\left(T_{x_{k}} L\right)
$$

so that their tensor product is canonically trivial. Given a relative spin structure for $\phi: L \rightarrow X$ the orientation at $u$ is determined by an isomorphism

$$
\operatorname{det}\left(D_{u}\right) \cong \mathbb{D}_{x_{0}, j_{0}}^{+} \otimes \mathbb{D}_{x_{1}, j_{1}}^{-} \otimes \ldots \otimes \mathbb{D}_{x_{d}, j_{d}}^{-},
$$


where each $j_{k} \in\{1,2\}$ depending on the type of end associated to $x_{k}$. The isomorphism (31) is determined by degenerating the surface with strip-like ends to a nodal surface. Thus each end $\epsilon_{e}, e \in \mathcal{E}\left(S_{v}\right)$ of a component $S_{v}$ with a node $q_{k}$ mapping to a selfintersection point is replaced by a disk $S_{v^{ \pm}(k)}$ with one end attached to the rest of the surface by a node $q_{k}^{ \pm}$. After combining the orientations on the determinant lines on $S_{v^{ \pm}(k)}$ with coherent orientations on the tangent spaces to the stable manifolds $W_{x_{k}}^{ \pm}$in the case of broken edges or semi-infinite edges $e \in \operatorname{Edge}(\Gamma), \ell(e)=\infty$, one obtains an orientation on the determinant line of the parameterized operator $\operatorname{det}\left(\tilde{D}_{u}\right)$ and so orientations on the regularized moduli spaces $\mathcal{M}_{\Gamma}(\phi, D)$. In particular the rigid component $\mathcal{M}(\phi, D)_{\rho}$ of the moduli space (that it, the component of expected dimension zero) inherits an orientation map

$$
\epsilon: \mathcal{M}(\phi, D)_{\rho} \rightarrow\{+1,-1\}
$$

comparing the constructed orientation to the canonical orientation of a point.

In general, Cieliebak-Mohnke perturbations [15] are not sufficient for achieving transversality if there are multiple markings on ghost bubbles. Indeed, if there exists a sphere component $S_{v} \subset S, v \in \operatorname{Vert}$ ( $(\Gamma)$ on which the map $\left.u\right|_{S_{v}}$ is constant and maps to the divisor so that $u\left(S_{v}\right) \subset D$, the domain $S_{v}$ may meet any number of interior leaves $T_{e} \subset T$. Adding an bulk leaf $T_{e^{\prime}}$ to the tree meeting $S_{v}$ increases the dimension of a stratum $\operatorname{dim} \mathcal{M}_{\Gamma}(\phi, D)$, but leaves the expected dimension $\operatorname{Ind}\left(D_{u}\right), u \in \mathcal{M}_{\Gamma}(\phi, D)$ unchanged so $\mathcal{M}_{\Gamma}(\phi, D)$ is not of expected dimension for some types $\Gamma$. A similar phenomenon occurs in the immersed case for constant disks $u \mid S_{v}: S_{V} \rightarrow X$ mapping to the self-intersection points $x, \bar{x}$ of the Lagrangian $\phi: L \rightarrow X$. We call a holomorphic treed disk $u: C \rightarrow X$ uncrowded if each such ghost component $S_{v} \subset S$ meets at most one bulk leaf, $T_{e} \cap S_{v} \neq \emptyset$. Cieliebak-Mohnke perturbations $\underline{P}=\left(P_{\Gamma}\right)$ suffice to make strata for uncrowded types of expected dimension at most one regular. On the other hand, the perturbations $P_{\Gamma}$ are chosen to satisfying the (Locality Axiom) so that for any crowded component $\mathcal{M}_{\Gamma}(\phi)$ there exists a non-empty uncrowded component $\mathcal{M}_{\Gamma^{\prime}}(\phi)$ of the moduli space obtained by forgetting all but one of the interior markings on such ghost bubbles $S_{v}$. Since combinatorial types $\Gamma^{\prime}$ with sphere bubbles are codimension two, such configurations $u: C \rightarrow X$ do not appear in the components of the moduli space $\overline{\mathcal{M}}(\phi, D)$ of expected dimension at most one.

The construction of regular perturbations now proceeds inductively by combinatorial type. More precisely, suppose that $d, e \geq 0$ are integers. Let $\Gamma$ be an uncrowded type of stable treed disk of expected dimension at most one with $d$ incoming edges and $e$ edges in total, and suppose that regular, stabilizing perturbation data $P_{\Gamma^{\prime}}$ have been chosen for all uncrowded boundary strata with $d^{\prime} \leq d$ incoming edges and $e^{\prime} \geq e$ total edges, 
with $\left(d^{\prime}, e^{\prime}\right) \neq(d, e)$. In particular, perturbations have been chosen for any boundary stratum $\mathcal{U}_{\Gamma^{\prime}} \subset \overline{\mathcal{U}}_{\Gamma}$. By the gluing construction, we obtain regular perturbation data on a neighborhood $V_{\Gamma}$ of the boundary of $\overline{\mathcal{U}}_{\Gamma}$. Denote by $\mathcal{P}_{\Gamma}=\left\{P_{\Gamma}\right\}$ the Baire space of perturbations that agree with the given perturbations on $V_{\Gamma}$.

Theorem 4.4 Let $\Gamma$ be a combinatorial type of adapted holomorphic treed disk of expected dimension at most one. There exists a comeager subset $\mathcal{P}_{\Gamma}^{\text {reg }}$ of the space $\mathcal{P}_{\Gamma}$ such that for $P_{\Gamma} \in \mathcal{P}_{\Gamma}^{\text {reg }}$,

(a) (Transversality) every element of $\mathcal{M}_{\Gamma}(\phi, D)$ is regular;

(b) (Compactness) the closure $\overline{\mathcal{M}}_{\Gamma}(\phi, D)$ is compact and contained in the adapted, uncrowded locus;

(c) (Boundary description) The boundary of $\overline{\mathcal{M}}_{\Gamma}(\phi, D)$ is a union of components $\mathcal{M}_{\Gamma^{\prime}}(\phi, D)$ where either $\Gamma^{\prime}$ is a type with an edge of a length zero, a broken edge connecting two disk components, or a broken semi-infinite edge corresponding to bubbling off a Morse trajectory;

(d) (Tubular neighborhoods) each uncrowded stratum $\mathcal{M}_{\Gamma}(\phi, D)$ of dimension zero has a tubular neighborhood of dimension one in any adjoining uncrowded strata of one higher dimension;

(e) (Orientations) the uncrowded strata $\mathcal{M}_{\Gamma}(\phi, D)$ of formal dimension one or two are equipped with orientations satisfying the standard gluing signs for inclusions of boundary components; in particular denote by $\epsilon(u) \in\{ \pm 1\}$ the orientation sign associated to the zero-dimensional moduli spaces $\mathcal{M}(\phi, D)_{\rho}$.

We will not give a complete proof, since the arguments are very similar to those for the embedded case as in [15], [16], but rather sketch the arguments. The transversality for generic perturbations is a consequence of the Sard-Smale theorem. Given integers $k, p$ determining the Sobolev class as in the proof of Proposition 3.2 and some local trivialization of the universal curve $\mathcal{U}_{\Gamma}^{i} \rightarrow \mathcal{M}_{\Gamma}^{i}$ denote by $\mathcal{M}_{\Gamma}^{\text {univ, } i}(\phi, D)$ the universal moduli space

$$
\begin{aligned}
& \mathcal{M}_{\Gamma}^{\text {univ }, i}(\phi, D)=\left\{\left(C, u, \partial u, u_{1}, u_{2}, P_{\Gamma}\right)\right. \\
& \in \mathcal{M}_{\Gamma}^{i} \times \operatorname{Map}\left(S^{\circ}, X\right)_{k, p} \times \operatorname{Map}\left(\partial S^{\circ}, L\right)_{k, p} \times \operatorname{Map}\left(T_{1}, L\right)_{k, p} \\
&\left.\times \operatorname{Map}\left(T_{2}, L \times{ }_{\phi} L-\Delta_{L}\right)_{k, p} \times \mathcal{P}_{\Gamma}^{l}(X, D) \mid(*)\right\}
\end{aligned}
$$

where $l \gg k,(*)$ includes the conditions (16) together with the requirement that the interior edges $T_{e}$ map to the Donaldson hypersurface $D$; in the case with tangencies with the Donaldson hypersurface there are additional conditions at the nodes $w_{e}$ requiring 
given order of vanishing at $D$ as explained in [15], [16]. On a point $p$ in any nonconstant component $S_{v} \subset S, \mathrm{~d} u \mid S_{v} \neq 0$, orthogonality of any element $\eta$ of the cokernel $\operatorname{ker}\left(D_{u}^{*}\right)$ to variations of the almost complex structure $K \in T_{J_{\Gamma}} \mathcal{J}_{\Gamma}\left(J_{D}\right)$ implies that the cokernel element $\eta$ vanishes in an open neighborhood of $p$, and so everywhere on $S_{v}$ by unique continuation. Constant spheres $S_{v}, \mathrm{~d} u \mid S_{v}=0$ have surjective linearized operators $D_{u \mid S_{v}}$ since the trivial Cauchy-Riemann operator on the sphere is surjective. Finally constant disks $u \mid S_{v}: S_{V} \rightarrow X$ mapping with corners alternating $x, \bar{x}$ for some ordered self-intersection point $x \in X$ have surjective universal linearized operator since any cokernel element $\eta$ must be orthogonal to a variation of the Hamiltonian perturbation on an open subset, and so vanish again by unique continuation. The Sard-Smale theorem implies that the set $\mathcal{P}_{\Gamma}^{l, \text { reg, } i}$ of regular values of the projection onto $\mathcal{P}_{\Gamma}^{l, i}$ is comeager; we denote by

$$
\mathcal{P}_{\Gamma}^{\text {reg }}=\cap_{i} \mathcal{P}_{\Gamma}^{\text {reg, } i}
$$

so that if $P_{\Gamma} \in \mathcal{P}_{\Gamma}$ then every element of the moduli space $\mathcal{M}_{\Gamma}(\phi, D)$ is regular. A standard argument due to Taubes implies that for a comeager subset of the space of smooth perturbations the moduli space is transversally cut out. In particular, let $\Gamma$ be a type of expected dimension at most one and $\Gamma^{\prime}$ the type obtained by adding a tangency requirement at a marking or node. Then the moduli space $\mathcal{M}_{\Gamma^{\prime}}(\phi, D)$ is empty. On the other hand, $\Gamma$ and $\Gamma^{\prime}$ have the same underlying tree so by taking $P_{\Gamma} \in \mathcal{P}_{\Gamma^{\prime}}$ we may assume that every element of $\mathcal{M}_{\Gamma}(\phi, D)$ meets the Donaldson hypersurface $D$ transversally.

We sketch the proof of the compactness statement (b). The (Locality axiom) ensures that the moduli spaces $\mathcal{M}_{\Gamma}(\phi, D)$ corresponding to types $\Gamma$ with more than one interior marking $z_{i}^{\prime}, z_{k}^{\prime}$ on a ghost bubble $S_{v} \subset S, u_{*}\left[S_{v}\right]=0$ admit forgetful maps to moduli space $\mathcal{M}_{\Gamma^{\prime}}(\phi, D)$ with at most one ghost marking on each sphere bubble. Suppose the boundary of $\mathcal{M}_{\Gamma}(\phi, D)$ contains a configuration in a stratum $\mathcal{M}_{\Gamma^{\prime}}(\phi, D)$ containing a sphere bubble. If the sphere bubble is non-trivial, then the expected dimension of $\mathcal{M}_{\Gamma^{\prime}}(\phi, D)$ is at most -1 , and so empty. Thus all sphere bubbles occurring in the limiting configuration are ghost bubbles. On the other hand, any configuration of ghost bubbles has at least two markings to be stable, and so the configuration $\mathcal{M}_{\Gamma^{\prime}}(\phi, D)$ has intersection multiplicity $d(z)$ at least two with the Donaldson hypersurface at at least one point $z \in S$. But then $\mathcal{M}_{\Gamma^{\prime}}(\phi, D)$ is of negative expected dimension, and so empty. Thus the boundary of the locus of expected dimension at most one is the union of strata $\mathcal{M}_{\Gamma^{\prime}}(\phi, D)$ where $\Gamma$ is a stable type or a Morse trajectory has bubbled off.

To prove (d), each stratum for combinatorial type representing a breaking has a tubular neighborhood given by a gluing construction. In the case of immersed Floer theory 
with treed disks there are now three kinds of gluing constructions necessary for the construction of tubular neighborhoods. A detailed exposition of the necessary estimates may be found in Schwarz [53] for the Morse gluing, Abouzaid [1] for the disk gluing estimates; also see Schmäschke [50]. First, if two disks $S_{v_{k}}, v_{k} \in \operatorname{Vert}(\Gamma), k \in\{1,2\}$ are joined at a node corresponding to an edge $e \in \operatorname{Edge}(\Gamma)$ of length $\ell(e)=0$ in a treed holomorphic disk $u: C \rightarrow X$ at which the branch of $L$ is unchanged then a gluing procedure for holomorphic disks produces a one-dimensional family of treed holomorphic disks $u_{\varkappa}: C_{\varkappa} \rightarrow X$ depending on a gluing parameter $\varkappa \in[0, \epsilon)$ for some small $\epsilon>0$. The gluing $u_{\varkappa}$ converges to the given configuration $u$ as $\varkappa \rightarrow 0$, and $C_{\varkappa}$ is obtained from $C$ by replacing the adjacent disks $S_{v^{\prime}}, S_{v^{\prime \prime}}$ with a single disk $S_{v}^{\prime}$. Second, if the branch of $L$ changes at the node $q_{k}$ so that $q_{k}$ maps to a self-intersection point $x_{k} \in \mathcal{I}^{\text {si }}(\phi)$ then a gluing construction for holomorphic strips with transversely intersecting Lagrangian boundary conditions produces a family $u_{\varkappa}: C_{\varkappa} \rightarrow X$ where again $C_{\varkappa}$ is obtained from $C$ by replacing adjacent disks $S_{v^{\prime}}, S_{v^{\prime \prime}}$ with a single disk $S_{v}^{\prime}$. Finally, for edges $e$ with infinite length $\ell(e)=\infty$ the gluing construction for Morse trajectories produces a family $u_{\varkappa}: C_{\varkappa} \rightarrow X$ converging to infinity, where $C_{\varkappa}$ is obtained from $C$ by replacing a broken segment $T_{e}=T_{e, 1} \cup T_{e, 2}$ with an unbroken one $T_{e}^{\prime}$.

The boundary description in Theorem 4.4 (c) follows from the description of the tubular neighborhoods (d) implies a description for the topological boundary of the union of one-dimensional strata. Any top-dimensional stratum $\overline{\mathcal{M}}_{\Gamma}(\phi)$ has boundary strata $\overline{\mathcal{M}}_{\Gamma^{\prime}}(\phi)$ corresponding (potentially) to Morse trajectories of length zero or broken Morse trajectories $u: T_{e} \rightarrow L$ of length $\ell(e)$ infinity. The former strata $\mathcal{M}_{\Gamma^{\prime}}(\phi, D)$ are called fake boundary components since there are two ways of desingularizing a configuration $u \in \mathcal{M}_{\Gamma^{\prime}}(\phi, D)$ : either by gluing the two adjacent holomorphic disks $u_{i}: S_{i} \rightarrow X, u_{k}: S_{k} \rightarrow X$ or by deforming a zero length Morse trajectory so that $u_{i}, u_{k}$ are connected by a segment $T_{e}$ of length $\epsilon>0$ instead of a node. On the other hand, the strata with a broken segment $u: T_{e} \rightarrow L, \ell(e)=\infty$ are true boundary components of the one-dimensional component of the moduli space $\cup_{i=1}^{k} \mathcal{M}_{\Gamma_{i}}(\phi, D)_{1}$ since in this case there is a single way of moving into the interior, by making the length $\ell(e)$ of that segment $T_{e}$ finite, see Figures 10 and 9. Because we chose the trajectories on the branched edges to be constant, for the unperturbed almost complex structure $J_{D}$ and Morse function $m$, once one has a fake boundary component $M_{\Gamma}(\phi)$ where a holomorphic disk breaks at a self-transverse intersection, it also contains a true boundary component $\mathcal{M}_{\Gamma^{\prime}}(\phi)$ with an infinite edge mapping to the same self-transverse intersection, since configurations with every possible edge length at the self-intersection occur. However, for the perturbed almost complex structure and Morse function $J_{\Gamma}, m_{\Gamma}$ 

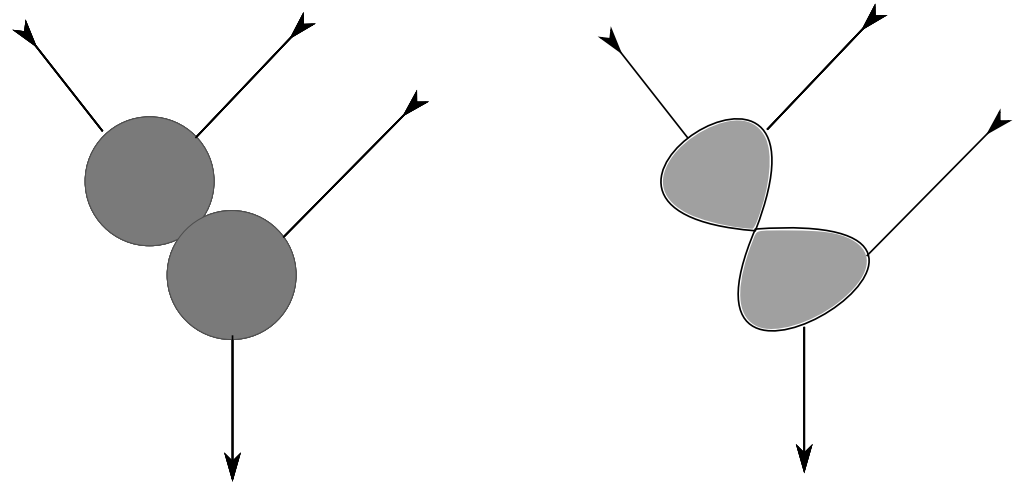

Figure 9: Fake boundary components with an unbranched resp. branched node
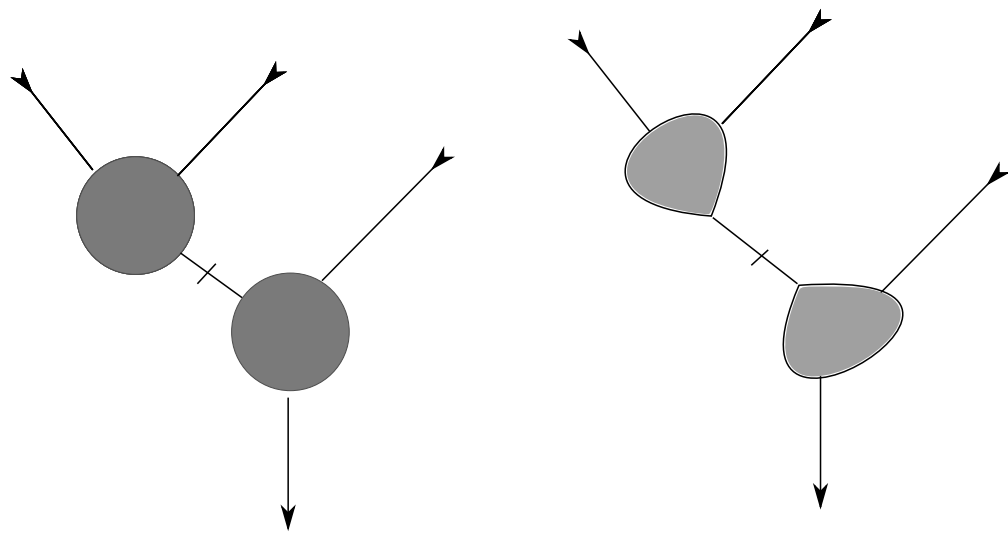

Figure 10: True boundary components with an unbranched resp. branched segment 
the perturbation depends on the length $\ell(e)$ of this trajectory, and so the existence of a non-empty stratum $\mathcal{M}_{\Gamma}(\phi)$ of a branched node with zero length $\ell(e)=0$ does not necessarily imply the existence of a non-empty stratum $\mathcal{M}_{\Gamma^{\prime}}(\phi)$ with infinite length.

Remark 4.5 (The exact case) An alternative construction of moduli spaces in the case of exact symplectic manifolds gives a definition of immersed Fukaya algebras over the integers. Let $\Gamma$ be a stable combinatorial type of nodal marked disks $S$ with no sphere components, with ordered boundary markings $z_{0}, \ldots, z_{d} \in \partial S$ in counterclockwise order around the boundary and unordered, possibly coinciding interior markings $z_{1}^{\prime}, \ldots, z_{c}^{\prime}$. That is, two such disks $S_{1}, S_{2}$ are isomorphic if there exists a biholomorphism $S_{1} \rightarrow S_{2}$ preserving the boundary marking $\underline{z}$ and preserving the interior markings $\underline{z}^{\prime}$ up to reordering. Let $\mathcal{M}_{\Gamma}^{\text {unord }}$ denote the moduli space of isomorphism classes of such disks, and $\overline{\mathcal{M}}_{d, c}^{\text {unord }}$ the union over combinatorial types $\Gamma$ with $d$ incoming leaves and $c$ interior markings:

$$
\overline{\mathcal{M}}_{d, c}^{\text {unord }}=\bigcup_{\Gamma} \mathcal{M}_{\Gamma}^{\text {unord }}
$$

Each moduli space $\mathcal{M}_{\Gamma}^{\text {unord }}$ is contractible, being identifiable with a subset of increasing elements $0=z_{1}<\ldots<z_{d-1}=1$ of the boundary markings and a subset $z_{1}^{\prime}, \ldots, z_{c}^{\prime} \in$ $\operatorname{int}(\mathbb{H})$ of the half-plane corresponding to the interior markings. The union $\overline{\mathcal{M}}^{\text {unord }}$ has a natural topology, similar to the moduli space of stable nodal disks $\overline{\mathcal{M}}_{d, c}$ with interior markings; in fact the latter admits a natural map to $\overline{\mathcal{M}}_{d, c}^{\text {unord }}$ obtained by collapsing all sphere components. Each moduli space $\mathcal{M}_{\Gamma}^{\text {unord }}$ is a smooth manifold equipped with a universal disk bundle

$$
\mathcal{U}_{\Gamma}^{\text {unord }} \rightarrow \mathcal{M}_{\Gamma}^{\text {unord }}
$$

Charts for $\mathcal{M}_{\Gamma}^{\text {unord }}$ near points $(C, z)$ where some of the markings coincide are produced using the algebra isomorphism $\mathbb{C}\left[z_{1}, \ldots, z_{n}\right]^{\Sigma_{n}} \cong \mathbb{C}\left[p_{1}, \ldots, p_{n}\right]$ where $\Sigma_{n}$ denotes the symmetric group on $\{1, \ldots, n\}$ and $p_{1}, \ldots, p_{n}$ are the elementary symmetric polynomials; taking spectra produces local charts for $\left(\mathbb{C}^{n}\right) / \mathbb{Z}_{n}$. Since each $\mathcal{M}_{\Gamma}^{\text {unord }}$ is contractible, the bundles $\mathcal{U}_{\Gamma}$ are trivial. Fix a trivialization $C \times \mathcal{M}_{\Gamma}^{\text {unord }}$. (The existence of a global trivialization is not necessary; if the bundle were only locally trivial we could perform the construction in each local trivialization and take the intersection of the comeager sets of perturbations.) Given a domain-dependent almost complex structure and Morse function $P_{\Gamma}=\left(J_{\Gamma}, m_{\Gamma}\right)$, let $J_{\Gamma}(C), m_{\Gamma}(C)$ denote the induced domain-dependent almost complex structure and Morse function on $S, T \subset C$. Denote 
the moduli space of adapted treed disks by

$$
\mathcal{M}_{\Gamma}^{\text {unord }}(\phi, D)=\left\{\begin{array}{l}
C, \underline{z}, u_{S}: S \rightarrow X, \partial u_{S}: \partial S \rightarrow L \\
u_{T_{1}}: T_{1} \rightarrow L, u_{T_{2}}: T_{2} \rightarrow L \times_{\phi} L \\
\bar{\partial}_{J_{\Gamma}, H_{\Gamma}} u_{S}=0,\left(\frac{d}{d s}+\operatorname{grad}\left(m_{\Gamma}\right)\right) u_{T_{2}}=0, u^{-1}(D)=\underline{z} \\
\left(\partial u_{S}\right)_{\partial S \cap T_{1}}=\left.\phi u_{T_{1}}\right|_{\partial S \cap T_{1}},\left(\partial u_{S}\right)_{\partial S \cap T_{2}}=\left.\phi u_{T_{1}}\right|_{\partial S \cap T_{2}}
\end{array}\right\}
$$

The same arguments that prove Theorem 4.4 show that for a comeager subset of perturbations $P_{\Gamma}$ the moduli space $\mathcal{M}_{\Gamma}^{\text {unord }}(\phi, D)$ is cut out transversally; for recursively

defined perturbation data the locus of the moduli space $\overline{\mathcal{M}}^{\text {unord, }<E}(\phi, D)$ of energy at most $E$ is compact. This ends the Remark.

\section{Holomorphic disks with self-tangent boundary condition}

In this section we construct moduli spaces of holomorphic curves for an immersed Lagrangian with a non-degenerate self-tangency. For simplicity we suppose that we are given a Lagrangian immersion $\phi: L \rightarrow X$ such that self-intersection points $L \times_{\phi} L-\Delta_{L}$ are transverse except for a single (up to reordering) self-intersection

$$
\left(x_{0}, x_{1}\right) \in L \times L, \quad \phi\left(x_{0}\right)=\phi\left(x_{1}\right)=x
$$

that has a non-degenerate self-tangency. In particular the dimension of the intersection of tangent spaces satisfies

$$
\operatorname{dim}\left(\left(D_{x_{0}} \phi\left(T_{x_{0}} L\right)\right) \cap\left(D_{x_{1}} \phi\left(T_{x_{1}} L\right)\right)\right)=1 .
$$

We assume that there exist local coordinates $q_{1}, \ldots, q_{n}, p_{1}, \ldots, p_{n}$ on $X$ near the self-intersection point $x$ with $x$ as the origin:

$$
q_{1}(x)=\ldots=q_{n}(x)=p_{1}(x)=\ldots=p_{n}(x)=0 .
$$

We also assume that the branches $L_{1}, L_{2}$ of $\phi(L)$ meeting $x$ are a plane and the product of a circular arc with a plane:

(34) $L_{1}=\left\{p_{1}=\ldots=p_{n}=0\right\} \quad L_{2}=\left\{p_{1}=\left(1-q_{1}^{2}\right)^{1 / 2}+1, q_{2}=\ldots=q_{n}=0\right\}$.

Lemma 2.8 shows that we may assume these conditions in any generic Maslov flow. We say that $x$ is a standard self-tangency if there exist such local coordinates and the almost complex structure $J$ is chosen to be the standard complex structure in these coordinates in a neighborhood of the self-tangency.

Once self-tangencies are allowed, one loses the exponential decay property at the selfintersections. Let $\Gamma$ be a combinatorial type of treed disk with immersed Lagrangian 
boundary condition and let $w \in C$ be a point at which a branched segment in $T_{2}$ meets the surface part, so that $w \in T_{2} \cap S$. Let

$$
\epsilon_{w}:[0, \pm \infty) \times[0,1] \rightarrow S
$$

be a holomorphic local coordinate on $S$ (with sign depending on whether the end is incoming or outgoing) with limit

$$
\lim _{\tau \rightarrow \pm \infty} \epsilon_{w}(\tau, t)=w, \quad \forall t \in[0,1] .
$$

Denote by $E_{w} \subset S$ the image of $[1, \infty) \times[0,1]$ under $\epsilon_{w}$. Denote by

$$
E_{w}\left[\tau_{0}\right]:=\epsilon_{w}\left(\left(\tau_{0}, \infty\right) \times[0,1]\right) \subset S
$$

the part of the end with $\tau>\tau_{0}$.

Lemma 5.1 (Exponential decay at self-intersections, similar to Proposition 4.6 in [23]) Let $u: C \rightarrow X$ be a holomorphic disk with boundary on a Lagrangian immersion $\phi: L \rightarrow X$ such that a point $z \in C$ maps to a self-intersection $x=\phi\left(x_{0}\right)=$ $\phi\left(x_{1}\right),\left(x_{0}, x_{1}\right) \in L^{2}$.

(a) If the self intersection $x$ is transverse then there exists a local coordinate $\epsilon_{w}(\tau+i t)$ near $z$ so that if $\tau>\tau_{0}$ then (omitting $\epsilon_{w}$ ) for some $\theta>0$,

$$
|u(\tau+i t)|=O\left(e^{-\theta \tau}\right)
$$

(b) If the self-intersection $x$ is a standard self-tangency then either

$$
|u(\tau+i t)|=O\left(e^{-\theta \tau}\right)
$$

as in the previous item or there exists a real number $c_{0}$ such that if $\tau>\tau_{0}$ then up to involution

$$
u(\tau+i t)=\left(\frac{2}{c_{0}+\tau+i t}, 0, \ldots, 0\right)+O\left(e^{-\theta \tau}\right)
$$

where $O\left(e^{-\theta \tau}\right)$ indicates exponential decay as in the first item.

Proof The first item is Floer's exponential decay estimate [29, Proof of Theorem 4], see also [28]. The second is also considered in Floer [29, Lemma 4.2], in the context of a study of the Fredholm index of the linearized operator; see also Ekholm-Etnyre-Sullivan [23, Lemma 4.6]. The proof of the statement here is, as in Floer [29], simpler than the treatment in Ekholm-Etnyre-Sullivan [23], since we do not perturb the boundary condition $\phi(L)$ near the self-tangency $x$. The self-tangent boundary conditions in $\mathbb{C}$ given in the first coordinate in (34) may be transformed to affine linear boundary 
conditions via the conformal transformation $u \mapsto 1 / u$. That is, the map $v(z)=1 / u_{1}(z)$ is holomorphic and has boundary conditions given by $\operatorname{Im}(v)(\tau)=0, \operatorname{Im}(v)(\tau+i)=1 / 2$. Composing with the holomorphic map $v \mapsto \exp (-2 \pi v)$ we obtain a finite energy holomorphic map with boundary conditions $\mathbb{R}$. By definition $v(z)$ has a limit as $\tau \rightarrow 0$ in the local coordinate $z=\exp (\pi(\tau+i t))$, and must be given by a map of the form

$$
\exp (-2 \pi v(z))= \pm \exp \left(2 c_{0}\right) z+O\left(z^{2}\right)
$$

Assuming the positive sign we have

$$
\begin{aligned}
u_{1}(t, \tau) & =v(t, \tau)^{-1} \\
& = \pm\left(\ln \left(\exp \left(\left(c_{0}+t+i \tau\right) / 2+O\left(e^{-\tau}\right)\right)\right)\right)^{-1} \\
u(t, \tau) & =\left(\frac{2}{c_{0}+\tau+i t}, 0, \ldots, 0\right)+O\left(e^{-\theta \tau}\right)
\end{aligned}
$$

(where $\theta$ depends on the Kähler angles of the transverse components) defines a holomorphic map from a neighborhood of 0 in the half plane $\operatorname{Im}(z) \geq 0$ to $\mathbb{C}$ with boundary in $\mathbb{R}$ and has the claimed expansion.

Proposition 5.2 If $\phi: L \rightarrow X$ has only non-degenerate tangencies then near any $u: C \rightarrow X$ of combinatorial type $\Gamma$ the moduli space $\mathcal{M}_{\Gamma}(\phi)$ is cut out locally by a Fredholm map.

Proof The statement of the proposition is essentially that in Floer [29, Theorem 4a], who studies non-degenerate tangencies as a method for developing an index theorem for the transverse case, or Ekholm-Etnyre-Sullivan [23, Corollary 7.14]. For simplicity assume a unique self-tangent point at $y \in X$. Let $C$ be a tree disk of type given by $\Gamma$ and let $q_{1}, \ldots, q_{m} \in C$ be the set of nodes corresponding to maps to the self-tangent point. We assume that the universal curve $\mathcal{U}_{\Gamma} \rightarrow \mathcal{M}_{\Gamma}$ is equipped with a smooth varying system of local coordinates $\epsilon_{k}:[0,1] \times \mathbb{R} \rightarrow S$ near the nodes $q_{k}$ for any fiber $C \subset \mathcal{U}_{\Gamma}$. Given $\underline{c}=\left(c_{1}, \ldots, c_{m}\right) \in \mathbb{R}^{m}$, fix a smooth reference map of the form

$$
u_{\mathrm{ref}}^{\underline{c}}: V \rightarrow X, \quad z \mapsto\left(-2\left(z+c_{k}\right)^{-1}, 0, \ldots, 0\right)
$$

near the node $q_{k}$. Choose a time-dependent exponential map on the ends $\exp _{u, t}$ : $T_{x} X \rightarrow X$ so that

$$
\exp _{u, t=0}: T_{x} L_{1} \rightarrow L_{1}, \quad \exp _{u, t=1}: T_{x} L_{2}(0) \rightarrow L_{2}(0)
$$

in a neighborhood of the points $x_{0}, x_{1} \in L$ mapping to $x \in X$. For $p \geq 2, k \geq 1, k p>2$ and real number $\lambda$ let $\Omega\left(C, u^{*} T X\right)_{k, p, \lambda}$ be the Sobolev space of $k, p$ maps defined using the measure $e^{\lambda \tau}$ on the strip-like end in the coordinates $z=\tau+i t$. Denote by 
$\operatorname{Map}_{k, p, \lambda}^{\Gamma}(C, X)$ be the space of maps $u$ of combinatorial type $\Gamma$ of Sobolev class $W_{\text {loc }}^{k, p}$ of the form $\exp _{u_{\text {ref }}^{c}}(\xi)$ for $\xi \in \Omega^{0}\left(C, u^{*} T X\right)_{k, p, \lambda}$ for some reference map $u_{\text {ref }}^{c}$. The class of maps that exponentially decay to one of these reference maps is independent of choice of reference map and local coordinates on the ends. Locally in a neighborhood of a map $(u, \partial u):(C, \partial C) \rightarrow(X, L)$ the moduli space $\mathcal{M}_{\Gamma}(\phi, D)$ is cut out by a Fredholm section of a Banach vector bundle. Given some local trivialization $\mathcal{U}_{\Gamma}^{i} \rightarrow \mathcal{M}_{\Gamma}^{i} \times C$ of the universal treed disk there is a bundle with base

$$
\mathcal{B}_{\Gamma}^{i}=\left\{\begin{array}{c}
\left(C, u, \partial u, u_{T_{1}}, u_{T_{2}}\right) \in\left(\begin{array}{c}
\mathcal{M}_{\Gamma}^{i} \times \operatorname{Map}_{k, p, \lambda}\left(S^{\circ}, X\right) \times \operatorname{Map}_{k, p, \lambda}\left(\partial S^{\circ}, L\right) \\
\times \operatorname{Map}_{k, p}\left(T_{1}, L\right) \times \operatorname{Map}_{k, p}\left(T_{2}, L\right) \\
\phi \circ \partial u_{S}=\left.u_{S}\right|_{\partial S},\left.\quad \partial u\right|_{\partial S^{\circ} \cap T_{1}}=u_{T_{1}}\left|S \cap T_{1}, \quad \partial u\right|_{\partial S^{\circ} \cap T_{1}}=u_{T_{1}} \mid S \cap T_{1}
\end{array}\right.
\end{array}\right\}
$$

of maps of Sobolev class $k, p$ with Sobolev weight $\lambda$ together with a treed disk of type $\Gamma$. Local charts for $\mathcal{B}_{\Gamma}$ can be constructed using geodesic exponentiation for some metric for which $L$ is totally geodesic; such a metric exists as long as the selfintersections of $L$ are self-transverse. We suppose for simplicity of notation that there is a single self-tangent node $q_{k}$ with $u\left(q_{k}\right)=x$. Consider the map

$$
\begin{aligned}
T_{c} \mathbb{R} \times \Omega^{0}\left(u^{c, *} T X,\left(\partial u^{c, *} T L\right)\right)_{k, p, \lambda} \rightarrow \operatorname{Map}_{k, p, \lambda}(S, X) & \\
(\delta c, \xi) & \mapsto \exp _{u^{c+\delta c}}\left(\Pi_{\delta c} \xi\right)
\end{aligned}
$$

where

$$
\Pi_{\delta c}: \Omega^{0}\left(u_{c, *} T X,\left(\partial u^{c, *} T L\right)\right)_{k, p, \lambda} \rightarrow \Omega^{0}\left(u_{c+\delta c, *} T X,\left(\partial u^{c+\delta c, *} T L\right)\right)_{k, p, \lambda}
$$

denotes parallel transport along the path of reference maps $u^{c+t \delta c}$. The fiber of the bundle $\mathcal{E}_{\Gamma}$ over some map $u$ is the vector space

$$
\mathcal{E}_{\Gamma, u}^{i}:=\Omega^{0,1}\left(S^{\circ}, u_{S}^{*} T X\right)_{k-1, p} \oplus \Omega^{1}\left(T_{1}, u_{T_{1}}^{*} T L\right)_{k-1, p} \oplus \Omega^{1}\left(T_{2}, u_{T_{2}}^{*} T L^{2}\right)_{k-1, p} .
$$

Local trivializations may be constructed using Hermitian parallel transport using the metric used to construct the charts for the base. The Fredholm map cutting out the moduli space locally is then

$$
\mathcal{F}_{\Gamma}^{i}: \mathcal{B}_{\Gamma}^{i} \rightarrow \mathcal{E}_{\Gamma}^{i},\left(u_{S}, u_{T_{1}}, u_{T_{2}}\right) \mapsto\left(\bar{\partial} u_{S}, \frac{d}{d s} u_{T_{1}}+\operatorname{grad}\left(m\left(u_{T_{1}}\right)\right), \frac{d}{d s} u_{T_{2}}\right) ;
$$

Here we use the assumption that the complex structure $J_{\Gamma}: \mathcal{U}_{\Gamma} \rightarrow J_{\tau}(X)$ is the standard one $J_{0}(z)=i z$ in a neighborhood of the self-tangency $x_{0}$ in the given local coordinates. This assumption implies that the one-form $\bar{\partial} u_{S}$ has the required exponential decay independent of the choice of reference map. The choice of Sobolev weight $\lambda \neq 0$ smaller than any of the angles in (53) guarantees that the linearized operator $\tilde{D}_{u}$ is 
Fredholm. As before, after imposing a finite energy condition $E(u), E$ any holomorphic treed disk $u: C \rightarrow X$ has a well-defined limit $\lim _{s \rightarrow \infty} u\left(\epsilon_{k}(s, t)\right)$ along any strip-like end $\epsilon_{k}$ converging to a node $q_{k}$ mapping to a self-intersection point $x_{0} \in \phi\left(L \times{ }_{\phi} L-\Delta_{L}\right)$. The required moduli space $\mathcal{M}_{\Gamma}(\phi)$ is then the subset of the zero set $\mathcal{F}_{\Gamma}^{i,-1}(0)$ of the resulting Fredholm map satisfying the matching condition at the nodes (quotiented by the group of automorphisms of $C$, if the domain $C$ is unstable.)

The moduli spaces with self-tangent boundary can be regularized as before using stabilizing divisors. Using Lemma 4.1 choose a Donaldson hypersurface $D \subset X$ so that $\phi(L) \subset X$ is exact in $X-D$. Requiring the bulk leaves $T_{e}$ to map to $D$, and any component of $u^{-1}(D)$ containing an bulk leaf, we may assume that the domains have stable surface part $S \subset T$ and use domain-dependent almost complex structures and Morse functions. As in Theorem 4.4, for a comeager subset $\mathcal{P}_{\Gamma, i}^{\text {reg }}$ of domain-dependent perturbations the moduli space of treed holomorphic disks $u: C \rightarrow X$ is transversally cut out for fibers $C \subset \mathcal{U}^{i}$ in a local trivialization $\mathcal{U}_{\Gamma}^{i} \rightarrow \mathcal{M}_{\Gamma}^{i}$ of the universal curve; taking the finite intersection $\mathcal{P}_{\Gamma}^{\text {reg }}=\cap_{i} \mathcal{P}_{i, \Gamma}^{\text {reg }}$ of these comeager sets produces the desired comeager subset of regular perturbations.

There is also a moduli space denoted $\mathcal{M}_{\Gamma}^{e}(\phi)$ with "exponential decay" at the selftangency, which will be empty for regular perturbations. The moduli space $\mathcal{M}_{\Gamma}^{e}(\phi)$ is constructed in the same way, but using a constant reference function $u^{\mathrm{ref}}(z)=\phi(y)$ near the self-tangency nodes $q_{k}$ instead of the reference function in (36). For generic choices of perturbation data $P_{\Gamma}$, the exponential decay moduli spaces $\mathcal{M}_{\Gamma}^{e}(\phi)$ are, by construction, of lower dimension than the moduli spaces $\mathcal{M}_{\Gamma}(\phi)$ that allow the reference function (36). Thus,

Proposition 5.3 (Similar to [23, Remark 9.20]) Let $\Gamma^{e}$ denote a combinatorial type of treed adapted holomorphic disk with exponential decay at a tangency. Let $\Gamma$ be the corresponding combinatorial type of treed holomorphic disk without the self-tangency requirement. If $\mathcal{M}_{\Gamma}(\phi, D)$ has expected dimension zero, then for regular perturbation data the moduli space $\mathcal{M}_{\Gamma^{e}}^{e}(\phi, D)$ is empty.

Given a relative spin structure for the self-tangent immersion, orientations for the moduli spaces $\overline{\mathcal{M}}(\phi, D)$ may be constructed as in Fukaya-Oh-Ohta-Ono [31], see also Wehrheim-Woodward [59], by bubbling off boundary value problems on disks with given paths $\gamma_{x}:[0,1] \rightarrow \operatorname{Lag}\left(T_{x} X\right)$. For $x=y \in X$ the self-tangent intersection point of $\phi: L \rightarrow X$, the natural gluing of the boundary value problems associated to the once-punctured disk with marking $y$ and boundary condition $\gamma_{y}$ and a marking $\bar{v}$ with boundary condition $\gamma_{\bar{v}}$ produces a boundary value problem on the disk with 
index one (since the Sobolev spaces are defined using a small negative Sobolev weight and so (after stabilization) kernel isomorphic to $T_{y} L \oplus \mathbb{R}$. For the self-tangent selfintersection, we require as part of the coherence axiom for orientations that $o_{y} \in$ $\operatorname{det}\left(D_{y}\right), o_{\bar{v}} \in \operatorname{det}\left(D_{\bar{v}}\right)$ should be defined so that the natural gluing map

$$
\mathbb{D}_{y, 2}^{+} \otimes \mathbb{D}_{y, 2}^{-} \rightarrow \mathbb{R}
$$

produces the standard orientation on the determinant of the one-dimensional index of the boundary condition where $\mathbb{D}_{y, 2}^{+}=\operatorname{det}\left(D_{y, 2}\right)$ and $\mathbb{D}_{y, 2}^{-}=\operatorname{det}\left(D_{y, 2}^{-}\right) \otimes \operatorname{det}\left(T_{y} L\right)$. The determinant line in (40) is an odd determinant line in the language of DeligneFreed [21], so that permuting it with other determinant lines produces signs that will contribute to the gluing sign for deformation of the Lagrangian boundary condition.

Compactness results follow from the discussion in Etnyre-Ekholm-Sullivan [23]. In particular [23, Theorem 11.2] construct compactified parametrized moduli spaces of holomorphic curves as the Lagrangian boundary condition develops a self-tangency in an isotopy $\phi_{t}, t \in[-\epsilon, \epsilon]$. Energy quantization holds uniformly in $t$ in the following sense:

Lemma 5.4 There $\hbar>0$ such that any non-constant holomorphic polygon $u: S \rightarrow X$ with boundary in $\phi_{t}$ except for those contained in a small neighborhood of the selftangency, so that any such holomorphic polygon not contained in a small neighborhood $U$ of $y$ has energy at least $E(u)>\hbar$.

Proof For holomorphic spheres or disks, the standard argument using the mean value inequality applies, see [43, Proposition 4.1.4]. On the other hand, since the distance between the branches of the Lagrangian is bounded from below away from the selfintersections, there exists a constant $c>0$ so that holomorphic polygons with a corner not contained in a neighborhood of the self-tangency contain a sub-domain of the form $u:[0, \infty] \times[0,1] \rightarrow X$ with the property that $d(u(T, 0), u(T, \infty))>c$. By the mean value inequality again, this is impossible if the energy is sufficiently small, since the derivative $\partial_{t} u(T, t)$ is bounded by a constant times the energy.

The lack of energy quantization for holomorphic strips in a neighborhood of the selftangency invalidates the argument in Gromov compactness [43, Proposition 4.7.1] used to prevent energy loss, see especially Step 2 in the proof in [43] which uses energy quantization. However, in the neighbourhood $U$ the branches $L_{0}, L_{1}$ of $\phi(L)$ are exact:

$$
\omega_{U}=\mathrm{d} \alpha,\left.\quad \alpha\right|_{L_{0}}=\mathrm{d} \zeta_{0}, \quad \alpha_{L_{1}}=\mathrm{d} \zeta_{1} .
$$


Energy loss can be ruled out using Stokes' theorem: For a sequence of holomorphic polygons $u_{\nu}: S_{\nu} \rightarrow X$ Gromov converging to $u: S \rightarrow X$ in the complement of the self-tangency $y$, let $V_{\nu}=u_{\nu}^{-1}(U)$ and $\left(\partial V_{\nu}\right)_{1}$ resp. $\left(\partial V_{\nu}\right)_{2} \cong \sqcup_{i=1}^{e_{\nu}}[0,1]$ the intersection of $\partial V_{\nu}$ with $\partial S$ resp. the closure of complement of $\partial S$ in $\partial V_{\nu}$. Write the intersection $\left(\partial V_{\nu}\right)_{1} \cap\left(\partial V_{\nu}\right)_{2}$ as a sequence $z_{0,1}, z_{1,1}, \ldots, z_{0, e}, z_{1, e}$ corresponding to the endpoints of the intervals in $\sqcup_{i=1}^{e_{\nu}}[0,1]$. Since $u_{\nu}$ converges to $u$, the number of ends $e_{\nu}$ converges to some $e$ for $\nu$ sufficiently large. The energy $E\left(u_{\nu} \mid u_{\nu}^{-1}(U)\right)$ is determined by

$$
\int_{u_{\nu}^{-1}(U)} u^{*} \omega=\int_{\left(\partial V_{\nu}\right)_{1}} u^{*} \alpha+\sum_{i=1}^{e_{\nu}} \zeta_{1}\left(z_{1, i}\right)-\zeta_{0}\left(z_{0, i}\right)
$$

which limits to $\int_{u^{-1}(U)} u^{*} \omega$. It follows that there is no energy loss at the self-tangency $y$ either.

Once one has excluded holomorphic maps with exponential decay to a self-tangency point, the moduli space of maps with a node mapping to a self-tangency splits as a disjoint union depending on whether the map approaches the tangency from the $x_{1}$ negative or positive, according to the sign in (35). We introduce two new symbols $v_{ \pm}$ and let

$$
\mathcal{I}^{\mathrm{si}}(\phi)=\left(L \times_{\phi} L-\Delta_{L}\right)-\{y\} \cup\left\{v_{+}, v_{-}\right\} .
$$

Then for $\underline{x} \subset \mathcal{I}^{\mathrm{c}}(\phi) \cup \mathcal{I}^{\text {si }}(\phi)$ we denote by $\mathcal{M}(\phi, D, \underline{x})$ the subset of the moduli space $\mathcal{M}_{\Gamma}(\phi, D, \underline{x})$ with the given limits $\underline{x}$, which distinguishes between holomorphic maps approaching from $x_{1}$ negative or positive in the local model.

We define a moduli space of holomorphic treed disks by imposing the following condition at nodes: If $e \in \operatorname{Edge}(\Gamma)$ is an edge corresponding to a segment $T_{e}$ of $T$ that maps to the self-tangency in $\phi_{0}$, then the limits of the disks on either end of $T_{e}$ are opposite, that is, if $z_{ \pm}$are the endpoints of the segment $\lim _{z \mapsto z+} u(z)=v_{+}$(that is, $u(z)$ approaches $y$ from the left in the local model) then $\lim _{z \mapsto z_{-}} u(z)=v_{-}$and similarly if $\lim _{z \mapsto z_{+}} u(z)=v_{-}$(that is, $u(z)$ approaches $y$ from the left in the local model) then $\lim _{z \mapsto z_{-}} u(z)=v_{+}$. Thus, the holomorphic treed disk "passes through" the self-tangency. We also allow the tangency points $v_{ \pm}$as input or output. In the case that $\phi_{t}: L \rightarrow X$ is a Maslov flow with a self-tangency at $t=0$ generating two new self-intersection points, the labels specifying the limits at infinity are

$$
\mathcal{I}^{\mathrm{si}}\left(\phi_{0}\right)=\mathcal{I}^{\mathrm{si}}\left(\phi_{-\epsilon}\right) \cup\left\{v_{-}, v_{+}\right\}, \quad \mathcal{I}^{\mathrm{si}}\left(\phi_{\epsilon}\right)=\mathcal{I}^{\mathrm{si}}\left(\phi_{-\epsilon}\right) \cup\left\{v_{+}, \bar{v}_{+}, \bar{v}_{-}, v_{-}\right\} .
$$

In other words, each direction of the self-tangency evolves into two ordered selftangencies. 


\section{Morse model for immersed Floer cohomology}

In this section we apply the transversality results of the previous section to construct immersed Floer theory in the Morse model. The generators of the immersed Floer space are critical points of a Morse function on the Lagrangian together with ordered self-intersection points of the immersion: Let

$$
m: L \rightarrow \mathbb{R}
$$

be a Morse function. Define

$$
\begin{aligned}
\mathcal{I}^{\mathrm{c}}(\phi) & =\operatorname{crit}(m)=\{x \in L \mid \mathrm{d} m(x)=0\} \\
\mathcal{I}^{\mathrm{si}}(\phi) & =\left\{\left(x_{1}, x_{2}\right) \in L^{2} \mid \phi\left(x_{1}\right)=\phi\left(x_{2}\right), x_{1} \neq x_{2}\right\} \\
\mathcal{I}(\phi) & =\mathcal{I}^{\mathrm{c}}(\phi) \cup \mathcal{I}^{\mathrm{si}}(\phi) \cup\left\{x^{\nabla}, x^{\nabla}\right\} .
\end{aligned}
$$

The set of self-intersections $\mathcal{I}^{\text {si }}(\phi)$ has a natural involution obtained by reversing the ordered pair:

$$
\mathcal{I}^{\mathrm{si}}(\phi) \rightarrow \mathcal{I}^{\mathrm{si}}(\phi), x=\left(x_{1}, x_{2}\right) \mapsto \bar{x}=\left(x_{2}, x_{1}\right) .
$$

Thus $\mathcal{I}(\phi)$ consists of the critical points of the Morse function together with two copies of each self-intersection point, plus two extra generators $x^{\nabla}, x^{\nabla} \in \mathcal{I}(\phi)$ used to construct strict units for the Fukaya algebra. We assume for simplicity $L$ is connected and $m: L \rightarrow \mathbb{R}$ has a single maximum $x_{+} \in \mathcal{I}^{\mathrm{c}}(\phi)$. The degrees are determined by

$$
\operatorname{deg}\left(x_{+}\right)=\operatorname{deg}\left(x^{\nabla}\right)=0, \quad \operatorname{deg}\left(x^{\nabla}\right)=-1 .
$$

Example 6.1 We consider an example of an immersion of the circle in the two-sphere with a pair of self-intersection points. Let $X \cong S^{2} \cong \mathbb{R}^{2} \cup\{\infty\}$. Consider the immersion of a circle $L \cong S^{1}$ as in Figure 11. Let $m: L \rightarrow \mathbb{R}$ be a Morse function with two critical points given by the maximum $x_{+}$and minimum $x_{-}$; the highest self-intersection point contributes two elements $v_{+}, \bar{v}_{+}$to $\mathcal{I}(\phi)$ where the ordering of the points in the self-intersection $L \times_{\phi} L$ is given by the direction of the arrow in the Figure. The second self-intersection point contributes elements $\bar{v}_{-}, v_{-}$similarly. Thus

$$
\mathcal{I}(\phi)=\mathcal{I}^{\text {si }}(\phi) \cup \mathcal{I}^{\mathrm{c}}(\phi) \cup\left\{x, x^{\nabla}\right\}=\left\{x_{+}, x_{-}, v_{+}, \bar{v}_{+}, \bar{v}_{-}, v_{-}, x, x^{\nabla}\right\} .
$$

This ends the example.

In order to obtain graded Floer cohomology groups a grading on the set of generators is defined as follows. Let $N$ be an even integer and $\operatorname{Lag}^{N}(X) \rightarrow \operatorname{Lag}(X)$ an $N$-fold Maslov cover of the bundle of Lagrangian subspaces as in Seidel [54]; we always

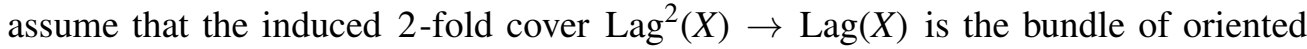




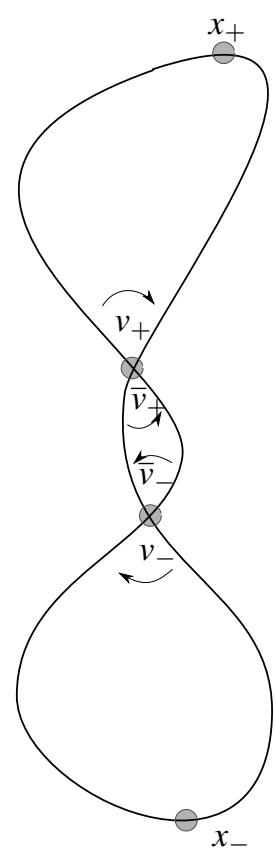

Figure 11: Generators for immersed Floer cohomology

Lagrangian subspaces. A grading of $\phi: L \rightarrow X$ is a lift $\phi^{N}$ to $\operatorname{Lag}^{N}(X)$ of the natural map $L \rightarrow \operatorname{Lag}(X), x \mapsto \operatorname{Im}\left(D_{x} \phi\right)$. Given such a grading, there is a natural $\mathbb{Z}_{N}$-valued map

$$
\mathcal{I}(\phi) \rightarrow \mathbb{Z}_{N}, \quad x \mapsto|x|
$$

obtained by assigning to any critical point the index $\bmod N$ and to any self-intersection point $\left(x_{-}, x_{+}\right) \in L$ the Maslov index of the image of any path from $\phi^{N}\left(x_{-}\right)$to $\phi^{N}\left(x_{+}\right)$ in $\operatorname{Lag}_{x}(X)$. Denote by $\mathcal{I}^{k}(\phi)$ the subset of $x \in \mathcal{I}(\phi)$ with $|x|=k$.

The moduli space of holomorphic disks is non-compact, and to remedy this the structure maps of the Fukaya algebra are defined over Novikov rings in a formal variable. Let

$$
\Lambda=\left\{\sum_{i=1}^{\infty} c_{i} q^{d_{i}} \mid c_{i} \in \mathbb{C}, d_{i} \in \mathbb{R}, \lim _{i \rightarrow \infty} d_{i}=\infty\right\}
$$

denote the universal Novikov field. The valuation by powers of $q$

$$
\operatorname{val}_{q}: \Lambda-\{0\} \rightarrow \mathbb{R}, \quad \sum_{i=1}^{\infty} c_{i} q^{d_{i}} \mapsto \min _{c_{i} \neq 0}\left(d_{i}\right)
$$

is well-defined and the Novikov ring

$$
\Lambda_{\geq 0}=\left\{f \in \Lambda \mid \operatorname{val}_{q}(f) \geq 0\right\}, \quad \text { resp. } \quad \Lambda_{>0}=\left\{f \in \Lambda \mid \operatorname{val}_{q}(f)>0\right\},
$$


is the subspace with non-negative resp. positive $q$-valuation. Let $\Lambda^{\times} \subset \Lambda$ be the subset with zero $q$-valuation.

The Floer cochain space is the free module over generators given by Morse critical points, self-intersection points, and the two additional generators from (44) necessary to achieve strict units. Let

$$
C F(\phi)=\bigoplus_{x \in \mathcal{I}(\phi)} \Lambda x
$$

The space of Floer cochains is naturally $\mathbb{Z}_{N}$-graded by

$$
C F(\phi)=\bigoplus_{k \in \mathbb{Z}_{N}} C F^{k}(\phi), \quad C F^{k}(\phi)=\bigoplus_{x \in \mathcal{I}^{k}(\phi)} \Lambda x .
$$

Put

$$
1_{\phi}:=x \vee C F(\phi) .
$$

The $q$-valuation on $\Lambda$ extends naturally to $C F(\phi)$ :

$$
\operatorname{val}_{q}: C F(\phi)-\{0\} \rightarrow \mathbb{R}, \quad \sum c(x) x \mapsto \min \left(\operatorname{val}_{q}(c(x)) .\right.
$$

We suppose that $L$ is equipped with a local system $y: \pi_{1}(L) \rightarrow \Lambda^{\times}$, and denote for any holomorphic treed disk $u: C \rightarrow X$ the holonomy of the local system around the boundary of the disks components in $C$ by $y(u) \in \Lambda^{\times}$. For regular perturbations define higher composition maps

$$
\mu_{d}: C F(\phi)^{\otimes d} \rightarrow C F(\phi)[2-d]
$$

on generators by

$$
\mu_{d}\left(x_{1}, \ldots, x_{d}\right)=\sum_{x_{0}, u \in \overline{\mathcal{M}}_{\Gamma}(\phi, D, \underline{x})_{\rho}}(-1)^{\ominus}(\sigma(u) !)^{-1} y(u) q^{E(u)} \epsilon(u) x_{0}
$$

where $\sigma(u)$ is the number of bulk leaves, $\epsilon(u) \in\{ \pm 1\}$ was defined in Theorem 4.4, and

$$
\varnothing=\sum_{i=1}^{d} i\left|x_{i}\right|
$$

Theorem 6.2 For any regular, coherent perturbation system $\underline{P}=\left(P_{\Gamma}\right)$ the maps $\left(\mu^{d}\right)_{d \geq 0}$ satisfy the axioms of a (possibly curved) $A_{\infty}$ algebra $C F(\phi)$ with strict unit $1_{\phi}=x \in C F(\phi)$. 
In particular, $\mu_{d}$ satisfy the $A_{\infty}$-associativity equations

$$
\begin{aligned}
0=\sum_{\substack{d_{1}, d_{2} \geq 0 \\
d_{1}+d_{2} \leq d}}(-1)^{d_{1}+\sum_{i=1}^{d_{1}}\left|x_{i}\right|} \mu^{d-d_{2}+1}\left(x_{1}, \ldots, x_{d_{1}},\right. \\
\left.\mu^{m}\left(x_{d_{1}+1}, \ldots, x_{d_{1}+d_{2}}\right), x_{d_{1}+d_{2}+1}, \ldots, x_{d}\right)
\end{aligned}
$$

for any $x_{1}, \ldots, x_{d} \in \mathcal{I}(\phi)$. Up to sign the relation (48) follows from the description of the boundary in Theorem 4.4 of the one-dimensional components, while the sign computation in [16] is independent of whether the Lagrangian is immersed or embedded. The element

$$
\mu_{0}(1) \in C F(\phi)
$$

is the curvature of the Fukaya algebra and has positive $q$-valuation $\operatorname{val}_{q}\left(\mu_{0}(1)\right)$. The Fukaya algebra $C F(\phi)$ is flat if $\mu_{0}(1)$ vanishes and projectively flat if $\mu_{0}(1)$ is a multiple of the identity $1_{\phi}$. Consider the sub-space of $C F(\phi)$ consisting of elements with positive $q$-valuation with notation from (46):

$$
C F(\phi)_{+}=\bigoplus_{x \in \mathcal{I}(\phi)} \Lambda_{>0} x
$$

Define the Maurer-Cartan map

$$
\mu: C F(\phi)_{+} \rightarrow C F(\phi), \quad b \mapsto \mu_{0}(1)+\mu_{1}(b)+\mu_{2}(b, b)+\ldots
$$

Let $M C(\phi)$ denote the space of weak solutions to the Maurer-Cartan space

$$
M C(\phi)=\left\{b \in C F^{\text {odd }}(\phi) \mid \mu(b)=W(b) 1_{\phi}, \quad W(b) \in \Lambda\right\} .
$$

The value $W(b)$ of $\mu(b)$ for $b \in M C(\phi)$ defines the disk potential

$$
W: M C(\phi) \rightarrow \Lambda \text {. }
$$

For $b \in C F(\phi)_{+}$define

$\mu_{d}^{b}\left(a_{1}, \ldots, a_{d}\right)=\sum_{i_{1}, \ldots, i_{d+1}} \mu_{d+i_{1}+\ldots+i_{d+1}}(\underbrace{b, \ldots, b}_{i_{1}}, a_{1}, \underbrace{b, \ldots, b}_{i_{2}}, a_{2}, b, \ldots, b, a_{d}, \underbrace{b, \ldots, b}_{i_{d+1}})$

For $b \in M C(\phi)$, the maps $\mu_{d}^{b}, d \geq 1$ form a flat $A_{\infty}$ algebra.

Remark 6.3 (Homotopy invariance) The homotopy type of the the immersed Fukaya algebra $C F(\phi)$ is independent of the choices of $J, m, D$ and $\underline{P}=\left(P_{\Gamma}\right)$ up to $A_{\infty}$ homotopy equivalences, by the immersed version of [16, Corollary 3.10] whose details we leave to the reader. The homotopy equivalences between $A_{\infty}$ algebras for perturbations $\underline{P}, \underline{P}^{\prime}$ induce maps between the Maurer-Cartan moduli spaces $M C(\phi, \underline{P}) \rightarrow M C\left(\phi, \underline{P}^{\prime}\right)$ 
so that Floer cohomology (as a collection of vector spaces $H F(\phi, b)$ over the MaurerCartan space $b \in M C(\phi))$ is an invariant of the Lagrangian immersion $\phi$.

The homotopy type of the immersed Fukaya algebra is also invariant under Hamiltonian diffeomorphism, but not exact isotopy. Given a Hamiltonian diffeomorphism $\psi: X \rightarrow$ $X$ any Donaldson hypersurface $D \subset X$ in the complement of $\psi(\phi(L))$ pulls back to a Donaldson hypersurface $\psi^{-1}(D) \subset X$ in the complement of $\phi(L)$. The domaindependent almost complex structure $J_{\Gamma}$ and Morse functions $m_{\Gamma}$ also pull back giving a bijection between treed holomorphic disks in the definition of the structure maps, so that $C F(\phi) \simeq C F(\psi \circ \phi)$. The immersed Fukaya algebra $C F(\phi)$ is not invariant under arbitrary exact isotopy $\phi_{t}: L \rightarrow X$ (even if the number of intersection points is preserved) since the areas of the holomorphic disks $u: C \rightarrow X$ with boundary in $\phi_{t}$ may change, destroying a solution to the Maurer-Cartan equation. See Example 6.7. This ends the Remark.

Example 6.4 We remind the reader of a basic computation of Floer cohomology in the Morse model for circles embedded in the two-sphere, which is a special case of the results of, for example, [32]: Let the symplectic manifold $X$ be the unit two-sphere $S^{2}$ in $\mathbb{R}^{3}$ with its standard symplectic form with volume $4 \pi$. The only embedded Lagrangian with non-trivial Floer cohomology is the equator, up to Hamiltonian isotopy. Indeed, consider an embedding $\phi: L=S^{1} \rightarrow X=S^{2}$ such that $\phi(L)$ separates $X$ into regions of areas $A_{-}, A_{+}$. To compute the embedded Floer theory let $m: S^{1} \rightarrow \mathbb{R}$ denote a Morse function on $L$ with a pair of critical points $x_{+}$resp. $x_{-}$the maximum resp. minimum of $m$. We have

$$
\mu_{0}(1)=q^{A_{+}} x_{+}+q^{A_{-}} x_{+}, \quad \mu_{1}\left(x_{-}\right)=q^{A_{+}} x_{+}-q^{A_{-}} x_{+} .
$$

If the geometric unit was the same as the strict unit, $b=0$ would give a weak MaurerCartan solution and the Floer cohomology of $\phi_{0}$ would be non-trivial if and only if $A_{+}=A_{-}$.

We explain why the distinction between strict and geometric units is immaterial in this case. The Floer theory has a weakly bounding cochain

$$
b=\left(q^{A_{+}}+q^{A_{-}}\right) x^{\nabla}
$$

For reasons of dimension $\mu_{n}(b, \ldots, b)$ vanishes for $b>1$ while

$$
\mu_{1}\left(x^{\nabla}\right)=x^{\nabla}-x_{+} .
$$

The graphs with a single edge and no vertex and weighting zero or infinity give the two terms on the right, while there are no terms with pseudoholomorphic disks since these are negative index. Hence

$$
\mu_{0}^{b}(1)=\mu_{0}(1)+\mu_{1}(b)=\left(q^{A_{+}}+q^{A_{-}}\right) x_{+}+\left(q^{A_{+}}+q^{A_{-}}\right)\left(x^{\nabla}-x_{+}\right)=\left(q^{A_{+}}+q^{A_{-}}\right) x^{\nabla} .
$$




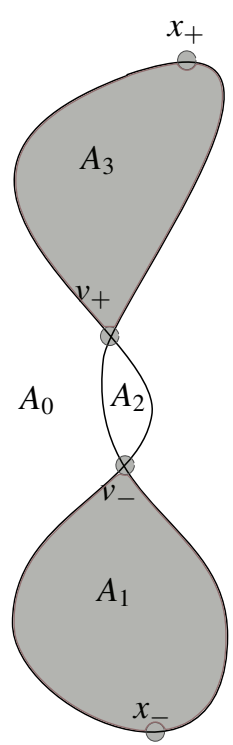

Figure 12: Teardrops obstructing Floer cohomology

With this choice of $b$ the Floer cohomology is again non-trivial iff $A_{+}=A_{-}$.

Example 6.5 We consider an immersion of the circle in the two-sphere with a pair of self-intersection points. Let $X$ be again the symplectic two-sphere $S^{2}$ and $L$ the circle $S^{1}$ with immersion $\phi: L \rightarrow X$ as shown in Figure 11. For the example shown in 6.1, recall that $v_{+}, \bar{v}_{+}$and $\bar{v}_{-}, v_{-}$are the generators of the Floer cochains created by the new self-intersection points while $x_{+}, x_{-}$are the maximum, resp. minimum of the Morse function, and $x \nabla, x^{\nabla}$ are the additional generators added to obtain strict units. The self-intersection points divide $\phi(L)$ into regions of areas $A_{1}, A_{2}, A_{3}$ while the exterior has area $A_{0}$. The curvature of the Fukaya algebra is, for some choices of trivializations of the determinant lines,

$$
\mu_{0}(1)=q^{A_{3}} v_{+}+q^{A_{1}} v_{-}+q^{A_{0}+2 A_{2}} x_{+}
$$

from contributions of the regions shown in Figure 12, with the final term the contribution from the "outside" of the circle. Thus $b=0$ is not a solution to the weak Maurer-Cartan equation.

We look for a weakly bounding cochain that "kills the teardrops". Working with the trivial local system $y \in \mathcal{R}(L)$ the first composition map has vanishing terms

$$
\mu_{1}\left(v_{-}\right)=0, \quad \mu_{1}\left(v_{+}\right)=0, \quad \mu_{1}\left(x_{+}\right)=0
$$


while

$$
\mu_{1}\left(\bar{v}_{+}\right)=-q^{A_{2}} v_{-}+q^{A_{3}} x_{+}, \quad \mu_{1}\left(\bar{v}_{-}\right)=-q^{A_{2}} v_{+}, \quad \mu_{1}\left(x_{-}\right)=q^{A_{1}} v_{-}+q^{A_{0}+2 A_{2}} x_{+} ;
$$

the last term arises from the holomorphic bigon $u: \mathbb{R} \times[0,1] \rightarrow X$ including the exterior that overlaps with itself in the region with area $A_{2}$. The negative signs depend on the choice of relative spin structures; here we take the determinant lines to be oriented by the tangent direction; then the relative spin structure on completion of the middle region $A_{2}$ is non-trivial and contributes a sign in the above formulas involving $A_{2}$. There are no other regions contributing to the $A_{\infty}$ structure maps involving $\bar{v}_{+}, \bar{v}_{-}$. If $\min \left(A_{1}, A_{3}\right)>A_{2}$ then in particular the element

$$
b_{0}=q^{A_{1}-A_{2}} \bar{v}_{+}+q^{A_{3}-A_{2}} \bar{v}_{-}
$$

has positive $q$-valuation and solves the Maurer-Cartan-like equation

$$
\mu_{0}^{b_{0}}(1) \in \operatorname{span}\left(x_{+}\right) .
$$

Indeed,

$$
\begin{aligned}
\mu_{0}^{b_{0}}(1) & =\mu_{0}(1)+\mu_{1}\left(b_{0}\right)+\mu_{2}\left(b_{0}, b_{0}\right)+\ldots \\
& =q^{A_{0}+2 A_{2}} x_{+}+q^{A_{1}} v_{+}+q^{A_{3}} v_{-}+q^{A_{1}-A_{2}} \mu_{1}\left(\bar{v}_{-}\right)+q^{A_{3}-A_{2}} \mu_{1}\left(\bar{v}_{+}\right) \\
& =q^{A_{0}+2 A_{2}} x_{+}+q^{A_{1}+A_{3}-A_{2}} x_{+}
\end{aligned}
$$

Ignoring the difference between the geometric and strict units the element above gives a weak Maurer-Cartan solution. To obtain an honest Maurer-Cartan solution as in the previous example we shift so that the zero-th composition map is a multiple of the strict unit:

$$
b=b_{0}+w x^{\nabla}, \quad w:=\left(q^{A_{0}+2 A_{2}}+q^{A_{1}+A_{3}-A_{2}}\right) .
$$

We claim that

$$
\mu_{0}^{b}(1)=w x^{\nabla}
$$

In case all the incoming leaves are labelled by elements $x \in \mathcal{I}(\phi)$ not equal to the minimum $x_{-}$, then any configuration $u: C \rightarrow X$ involving these elements with at least one vertex survives perturbation of the Morse function, almost complex structure, and metric. Indeed, the holomorphic disks $u: S \rightarrow X$ with boundary in $\phi(L)$ are invariant under perturbation of the almost complex structure, since the complex structure on the disk is unique up to diffeomorphism, and the condition on semi-infinite gradient trajectories on the leaves is that they flow up to the maximum. It follows that the only contributions to $\mu_{1}^{b_{0}}\left(w x^{\vee}\right)$ are those with at most one vertex in the corresponding graph, 
since otherwise the weighting parameter $\varkappa$ is free to vary and the configuration cannot be rigid. So as in the previous example,

$$
\begin{aligned}
\mu_{0}^{b}(1) & =\mu_{0}^{b_{0}}(1)+\mu_{1}^{b_{0}}\left(w x^{\nabla}\right) \\
& =w x_{+}+\mu_{1}^{0}\left(w x^{\nabla}\right) \\
& =w x_{+}+w\left(x^{\nabla}-x_{+}\right) \\
& =w x^{\nabla} .
\end{aligned}
$$

Hence

$$
b \in M C(\phi)
$$

We compute the Floer cohomology for this bounding cochain. For the same reason as in previous paragraph, we have $\mu_{1}^{b_{0}}=\mu_{1}^{b}$ and so we may ignore the difference between geometric and strict unit. The first composition map (that is, the differential) satisfies

$$
\mu_{1}^{b}\left(x_{+}\right)=0, \quad \mu_{1}^{b}\left(x_{-}+q^{A_{3}-A_{2}} \bar{v}_{-}\right)=\left(q^{A_{0}+2 A_{2}}-q^{A_{3}-A_{2}+A_{1}}\right) x_{+} .
$$

Since $\mu_{1}^{b}$ squares to zero, we must have

$$
\mu_{1}^{b}\left(q^{A_{2}} v_{-}+q^{A_{1}} x_{+}\right)=q^{A_{2}} \mu_{1}^{b}\left(v_{-}\right)=0, \quad \mu_{1}^{b}\left(q^{A_{2}} v_{+}\right)=q^{A_{2}} \mu_{1}^{b}\left(v_{+}\right)=0 .
$$

So $\mu_{1}^{b}\left(v_{-}\right)=\mu_{1}^{b}\left(v_{+}\right)=0$. Computing the cohomology of $\mu_{1}^{b}$ we have

$$
H F(\phi, b)= \begin{cases}\Lambda^{\oplus 2} & A_{0}+3 A_{2}=A_{3}+A_{1} \\ \{0\} & \text { otherwise. }\end{cases}
$$

We check that the computation is compatible with Hamiltonian displaceability. Note that the condition (49) implies

$$
\begin{aligned}
& A_{0}=A_{1}+A_{2}+A_{3}-4 A_{2} \\
& A_{1}=A_{0}+A_{2}+A_{3}+\left(2 A_{2}-2 A_{3}\right) \\
& A_{2}=A_{0}+A_{1}+A_{3}-2 A_{0}-2 A_{2} \\
& A_{3}=A_{0}+A_{1}+A_{2}+\left(2 A_{2}-2 A_{1}\right) .
\end{aligned}
$$

These equalities preclude any equality

$$
A_{i}>A_{j}+A_{k}+A_{l}, \quad i, j, k, l \text { distinct. }
$$

Equation (50) is equivalent to displaceability by Moser's theorem [46] since if one area $A_{i}$ is larger than the sum of the other three $A_{j}+A_{k}+A_{l}$ then there exists a Hamiltonian diffeomorphism $\psi: X \rightarrow X$ that moves the exterior of the largest region $X-A_{i}$ into its interior $\operatorname{int}\left(A_{i}\right)$. Thus the non-triviality of the Floer cohomology $H F(\phi, b)$ is consistent with non-displaceability. 


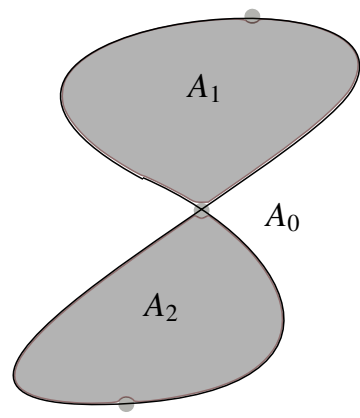

Figure 13: An example with vanishing Floer cohomology

Example 6.6 Consider an embedded circle with a single intersection point, dividing the sphere into regions $R_{0}$ ("outside"), $R_{1}$ and $R_{2}$ as in Figure 13, with areas $A_{0}, A_{1}, A_{2}$. Let $x_{+}$be the maximum, $v, \bar{v}$ the generators associated to the self-intersection points and $x_{-}$the minimum. The two lobes of the figure eight contribute to $\mu_{0}(1)=$ $\left(q^{A_{1}}+q^{A_{2}}\right) v$ with the same sign by invariance of the picture (up to deformation) under rotation by $\pi$. The first $A_{\infty}$ map for $b=0$ is given by

$$
\mu_{1}\left(x_{+}\right)=0, \quad \mu_{1}(v)=0, \quad \mu_{1}(\bar{v})=q^{A_{1}} x_{+}, \quad \mu_{1}\left(x_{-}\right)=q^{A_{2}} v .
$$

Indeed a configuration with an input $x_{+}$can never be rigid; there are two possible strips with input $v$ but these can be seen to cancel by the $A_{\infty}$ relations, or an explicit sign computation; the lobe with area $A_{2}$ contributes a holomorphic strip causing $\mu_{1}\left(x_{-}\right)=q^{A_{2}} v$. The higher composition maps involving $x_{-}$depend on a choice of perturbations. There is a choice for which one has a version of the divisor equation

$$
\mu_{d}\left(x_{-}, \ldots, x_{-}\right)=(d !)^{-1} \mu_{1}\left(x_{-}\right)=(d !)^{-1} q^{A_{2}} v
$$

explained in [48, Section 4.4]. The higher composition maps involving $v$ as input are trivial. A weakly bounding cochain (albeit) with zero $q$-valuation must have for some constant $c$

$$
b=\ln \left(-q^{A_{1}-A_{2}}\right) x_{-}+c v=-\pi i x_{-}+c v
$$

and therefore exists only if $A_{1}=A_{2}$ in which case

$$
\mu_{0}^{b}(1)=c x_{+} .
$$

The reader who is uncomfortable with the assertion on the divisor equation may take a local system whose holonomy around the lower lobe is -1 and take $b=0$. The Floer operator has

$$
\mu_{1}^{b}\left(x_{+}\right)=0, \quad \mu_{1}^{b}(v)=0, \quad \mu_{1}^{b}(\bar{v})=q^{A_{1}} x_{+}, \quad \mu_{1}^{b}\left(x_{-}\right)=-q^{A_{2}} v .
$$




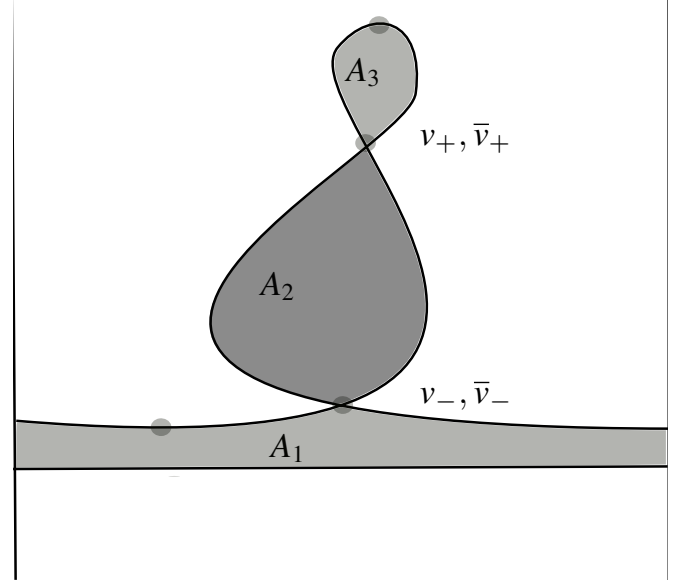

Figure 14: An exact isotopy of the zero section with obstructed Floer theory

Thus the Floer cohomology vanishes. On the other hand, $\phi(L)$ is non-displaceable if $A_{i}>A_{j}+A_{k}$ for some distinct $i, j, k$. We remark that Grayson [35] has conjectured that the mean curvature flow of $\phi$ converges to a point if $A_{1}=A_{2}$.

Example 6.7 The following is an example of Weinstein [61] of an exact isotopy of maps from the circle $S^{1}$ to the cylinder $T^{*} S^{1}$. The family includes immersions with both obstructed and unobstructed Floer cohomology. It also shows that nontriviality of immersed Floer cohomology is not an obstruction to displaceability by exact deformation. Let $\phi_{t}: S^{1} \rightarrow T^{*} S^{1}$ be an immersion of the form shown in Figure 14. If the areas satisfy the equality $A_{1}+A_{3}=A_{2}$, then $\phi_{1}(L)$ is an exact isotopy of $\phi_{0}(L)$ by standard Moser arguments. Let $\bar{v}_{-}, v_{-}$resp. $v_{+}, \bar{v}_{+}$be the generators of the Floer complex corresponding to the lower resp. higher self-intersection point (with bar if the ordered intersection point is odd)

$$
\mu_{0}(1)=q^{A_{3}} v_{+}, \quad \mu_{1}\left(\bar{v}_{-}\right)=q^{A_{2}} v_{+} .
$$

For unobstructedness the weakly bounding cochain is forced to equal

$$
b=q^{A_{3}-A_{2}} \bar{v}_{-} \in M C(\phi)
$$

as long as $A_{3}>A_{2}$ since the Maurer-Cartan space requires positive $q$-valuation $\operatorname{val}_{q}(b)>0$. This is impossible if $A_{2}=A_{1}+A_{3}$.

On the other hand, consider an exact isotopy of $\phi_{1}(L)$ with the property that $A_{1}$ is negative, so that $A_{3}>A_{2}$. In this case, $b$ is well-defined and the Floer cohomology is non-trivial. This ends the example. 


\section{Invariance for transverse self-intersection}

In this section we prove that Maslov flows not changing the number of self-intersection points leave the Floer cohomology invariant. This generalizes an argument in AlstonBau [3] which assumed a monotonicity condition on the Lagrangian. Since the structure maps in our setting are weighted by areas, we study how the areas of the disks contributing to the Fukaya algebra change as we deform the immersion under a Maslov flow, similar to the study in Angenent [5]. We show that immersed Lagrangian Floer theory possesses a canonical $\mathbb{R}$-grading, similar to the $\mathbb{Q}$-grading of the twisted sectors on orbifold quantum cohomology as in Chen-Ruan [14], (which is preserved only to leading order by the differential) which determines the change in the areas of the holomorphic polygons under Maslov flow.

First, we explain that for small times there exists a bijection between pseudoholomorphic disks with the given boundary condition.

Lemma 7.1 Let $\phi_{t}: L \rightarrow X, t \in[0, T]$ be an isotopy of Lagrangians with only self-transverse intersections and no triple intersections. For $t_{2}$ sufficiently close to $t_{1}$, there exists a diffeomorphism $\psi_{t_{1}}^{t_{2}}$ of $X$ mapping $\phi_{t_{2}}(L)$ to $\phi_{t_{1}}(L)$ and for which for any combinatorial type $\Gamma$ of treed disk the almost complex structure $\psi_{t_{1}}^{t_{2}} J_{\Gamma, t_{1}}$ is admissible. This diffeomorphism induces a bijection between moduli spaces $\mathcal{M}_{\Gamma}\left(\phi_{t_{1}}\right)$ and $\mathcal{M}_{\Gamma}\left(\phi_{t_{2}}\right)$, defined using $J_{\Gamma, t_{1}}$ resp. $\psi_{t_{1}}^{t_{2}, *} J_{\Gamma, t_{2}}$.

Proof As in Alston-Bao [3], the fact that there are only self-transverse intersections means that there exists a family of diffeomorphisms $\psi_{t_{1}}^{t_{2}}$ mapping $\phi_{t_{1}}(L)$ to $\phi_{t_{2}}(L)$. The assumption of transverse self-intersections and no triple self-intersections implies that $\cup_{t \in[0, T]}\left(\phi_{t}\left(L \times \phi_{t} L-\Delta_{L}\right)\right) \times\{t\}$ is an embedded submanifold of the product $X \times$ $[0, T]$. Standard arguments imply that there exists a tubular neighborhood $B_{\epsilon}\left(0, \mathbb{R}^{2 n}\right) \times$ $[0, T] \rightarrow X \times[0, T]$ of $\left\{x_{t}, t \in[0, T]\right\}$ so that the branches of $\phi_{t}(L)$ near any selfintersection are the images of $B_{\epsilon}\left(0, \mathbb{R}^{n} \times\{0\}\right)$ resp $B_{\epsilon}\left(0,\{0\} \times \mathbb{R}^{n}\right)$. The family $\psi_{t_{1}}^{t_{2}}$ is defined as the restriction of the flow of a vector field $v_{t}$ on $X \times[0, T]$ to $X \times\left\{t_{1}\right\}$, with $v_{t}$ defined so that (a) $D \pi v_{t}=\frac{d}{d t}$, where $\pi: X \times[0, T] \rightarrow[0, T]$ is the projection (b) $v_{t}$ is tangent to the submanifold of self-intersections $\cup_{t \in[0, T}\left(\phi_{t}\left(L \times_{\phi_{t}} L-\Delta_{L}\right)\right) \times\{t\}$ (c) $v_{t}$ is tangent to $\phi_{t}(L)$ away from the self-intersections $\phi_{t}\left(L \times_{\phi_{t}} L-\Delta_{L}\right)$. Such a vector field may be defined in stages, first in the tubular neighborhood of the self-intersections and then extended to $\phi_{t}(L)$ and $X$. Since tameness is an open condition, $\psi_{t_{1}}^{t_{2}, *} J_{\Gamma, t_{2}}$ is also tamed for $t_{1}$ sufficiently close to $t_{2}$, and similar for the stabilizing condition. The map $\psi_{t_{1}}^{t_{2}}$ induces the claimed bijection of moduli spaces of pseudoholomorphic treed disks. 
We give two definitions of the $\mathbb{R}$-grading. The first definition of the grading is an action-index difference.

Definition 7.2 Let

$$
\left(x_{-}, x_{+}\right) \in L^{2}, \quad u\left(x_{-}\right)=u\left(x_{+}\right)=x \in X
$$

be a self-intersection point. Choose a path

$$
\gamma_{x}:[-1,1] \rightarrow \operatorname{Lag}\left(T_{x} X\right), \quad \gamma( \pm 1)=D \phi_{x_{ \pm}}\left(T_{x_{ \pm}} L\right)
$$

as in (29). Consider the determinant map

$$
\operatorname{det}: \operatorname{Lag}\left(T_{x} X\right) \rightarrow \operatorname{Lag}\left(K_{x}^{-1}\right) \cong S^{1}
$$

given by the top exterior product. Let

$$
\mathrm{d} \theta \in \Omega^{1}\left(S^{1}\right), \quad \int_{S^{1}} \mathrm{~d} \theta=2 \pi
$$

denote the standard angular form that is circle-invariant. Define the action of the path

$$
a(x)=\int_{-1}^{1}\left(\operatorname{det} \gamma_{x}\right)^{*} \mathrm{~d} \theta / \pi
$$

obtained by integrating the pull-back of the angular form. If $\bar{x}=\left(x_{+}, x_{-}\right)$is the reversed self-intersection point denote by $\gamma_{\bar{x}}$ the opposite of the path $\gamma_{x}$ so that

$$
a(x)+a(\bar{x})=0, \quad i(x)+i(\bar{x})=n .
$$

Define

$$
\theta(x)=i(x)-a(x)
$$

independent of the choice of path $\gamma_{x}$.

Alternatively, the $\mathbb{R}$-grading may be given in terms of the sum of the Kähler angles of intersection of the Lagrangians.

Definition 7.3 Define an angle

$$
\theta_{1}=\min \left\{\theta \in(0,1) \mid e^{\pi i \theta} D \phi\left(T_{x_{-}} L\right) \cap D \phi\left(T_{x_{+}} L\right) \neq\{0\}\right\} .
$$

Replacing $D \phi\left(T_{x_{ \pm}} L\right)$ with their orthogonal complements in $T_{x} X$ and applying the construction iteratively to the complements, one obtains a basis

$$
\left\{y_{1}, \ldots, y_{n}\right\} \subset D \phi\left(T_{x_{+}} L\right)
$$

and angles

$$
\theta_{1}<\ldots<\theta_{n} \in(0,1)
$$


so that bases for the Lagrangian tangent spaces are given by

$$
D \phi\left(T_{x_{+}} L\right)=\operatorname{span}\left\{y_{1}, \ldots, y_{n}\right\}, \quad D \phi\left(T_{x_{-}} L\right)=\operatorname{span}\left\{e^{i \theta_{1}} y_{1}, \ldots, e^{i \theta_{n}} y_{n}\right\} .
$$

Then as in [3, Equation (3)]

$$
\theta(x)=n-\sum_{i=1}^{n} \theta_{i} \in(0, n) .
$$

We these definitions in place we prove a variational formula for the areas under Maslov flow. As in (11) suppose that the anticanonical bundle $K^{-1}$ is equipped with a connection $\alpha_{t}$ so that the infinitesimal variation $\cdot \phi_{t}$ is given by the corresponding oneform $\phi_{t}^{*} \alpha_{t}$ representing the connection on $\phi_{t}^{*} K^{-1}$. The following is a generalization of a formula of Angenent [5, bottom of page 1214] for holomorphic polygons with a single corner in the dimension two case, to arbitrary dimensions and polygons.

Lemma 7.4 The rate of change in the area of any family of rigid treed disks $u_{t}: C \rightarrow X$ with boundary in $\phi_{t}(L)$ and with vertices at infinity $z_{0}, \ldots, z_{d}$ (with only $z_{0}$ outgoing) mapping to intersection points $x_{0}, \ldots, x_{d} \in \mathcal{I}\left(\phi_{t}\right)$ is

$$
\frac{d}{d t} \int_{S} u_{t}^{*} \omega_{t}=\sum_{k>0} \theta\left(x_{k}\right)-d+2-\theta\left(x_{0}\right)
$$

with $\theta\left(x_{k}\right):=i\left(x_{k}\right)$ if $x_{k} \in \mathcal{I}^{c}\left(\phi_{t}\right)$.

Remark 7.5 If there are no self-intersections at the corners $z_{k}$ and no incoming markings $d=0$ the right-hand side is simply $2-i\left(x_{0}\right)$. For example, in the case of a single outgoing marking with output $x_{0}$ so that $i\left(x_{0}\right)=0$ each disk in the equation is Maslov index $I\left(u_{t}\right)=2$, while if only Maslov index two disks appear then the Fukaya algebra is projectively flat, that is, $\mu_{0}(1) \in \operatorname{span}\left(1_{\phi_{t}}\right)$.

Proof of Lemma We wish to put ourselves in the situation where there are no branch changes in the boundary condition, by adding additional disks at each of the branch changes. For simplicity we consider a configuration $C=S \cup T$ with a single disk $S$ with $d+1$ leaves $T=T_{0} \cup \ldots T_{d}$ with points at infinity $z_{0}, \ldots, z_{d} \in C$ mapping to self-intersection points $x_{0}, \ldots, x_{d} \in \mathcal{I}^{\text {si }}(\phi)$. Let $\tilde{S}$ be the disk equipped with complex bundle $E$ with totally real Lagrangian boundary condition $F$ obtained by gluing together $S$ equipped with the bundle pair $\left(u^{*} T X,(\partial u)^{*} T L\right)$ with the disks $S_{0}, \ldots, S_{d}$ equipped with bundles $T_{\phi\left(x_{i}\right)} X$ and boundary conditions corresponding to the paths

$$
\gamma_{x_{k}}:(0,1) \rightarrow \operatorname{Lag}\left(T_{\phi\left(x_{k}\right)} X\right), \quad j=0, \ldots, d .
$$




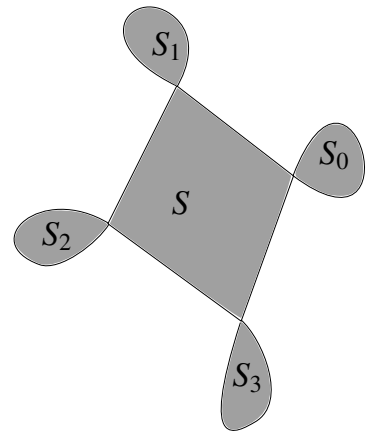

Figure 15: Gluing a polygon with once-punctured disks

See Figure 15; note that the capping disks $S_{0}, \ldots, S_{d}$ are not mapped to $X$ but rather are only equipped with bundle pairs. First assume that all vertices of $S$ map to selfintersections of $\phi$. The bundle $u_{t}^{*} K^{-1}$ extends to a bundle on $\bar{S}$ obtained by taking top exterior powers. The one-form $u_{t}^{*} \alpha$ extends to a one-form $\bar{\alpha}_{t}$ on the extension; via the given trivialization of the anticanonical bundle $\phi_{t}^{*} K^{-1}$ over the Lagrangian these connection one-forms become ordinary one-forms still denoted $\bar{\alpha}_{t} \in \Omega^{1}(\bar{S})$ on the base. Since the Maslov index of the glued problem $(E, F)$ is the winding number of the boundary condition,

$$
\int_{\partial \bar{D}} \overline{\alpha_{t}}=I(E, F)
$$

Since $\mu_{d}$ is a map of degree $2-d$, the Maslov index of any rigid disk contributing to $\mu_{d}$ must be the shifted difference in degrees

$$
I(E, F)=-i\left(x_{0}\right)+\sum_{i=1}^{d} i\left(x_{i}\right)-d+2 .
$$

On the other hand, the integral of $\overline{\alpha_{t}}$ around the boundary of any of the disks $S_{i}$ is by (51) equal to $\pm a_{t}\left(x_{i}\right)$, with a minus sign for the self-intersection point that is outgoing. Putting everything together the integral of the connection one-form $\alpha$ around the boundary $\partial S$ of the disk is

$$
\begin{aligned}
\sum_{i=0}^{d} \int_{\partial S} u_{t}^{*} \alpha & =\int_{\partial \bar{D}} \overline{\alpha_{t}}+a_{t}\left(x_{0}\right)-\sum_{i=1}^{d} a_{t}\left(x_{i}\right) \\
& =-i\left(x_{0}\right)+\sum_{i=1}^{d} i\left(x_{i}\right)-d+2+a_{t}\left(x_{0}\right)-\sum_{i=1}^{d} a_{t}\left(x_{i}\right) \\
& =\sum_{i=1}^{d} \theta_{t}\left(x_{i}\right)-\theta_{t}\left(x_{0}\right)-d+2 .
\end{aligned}
$$


The case that some of the points at infinity map to critical points $\mathcal{I}^{\mathrm{c}}(\phi)$ is similar, using the fact that each element $x_{k} \in \mathcal{I}(\phi)$ of index $i\left(x_{k}\right)$ that is not a self-intersection point cuts down the dimension of the moduli space by dimension $i\left(x_{k}\right)$. For the case that the configuration $C$ has several disk components, the computation follows by considering each disk separately and taking the sum.

The following Euler flow on Floer cochains cancels out the change in the areas of the disks under mean curvature flow of the immersion. Combine the $\mathbb{R}$-gradings on self-intersection points and critical points by

$$
\mathcal{I}(\phi) \rightarrow \mathbb{R}, \quad x \mapsto|x|:= \begin{cases}\theta(x) & x \in \mathcal{I}^{\mathrm{si}}(\phi) \\ \operatorname{dim}\left(W_{x}^{-}\right) & x \in \mathcal{I}^{\mathrm{c}}(\phi)\end{cases}
$$

where $W_{x}^{-}$is the unstable manifold of $\operatorname{grad}(m)$ at $x$.

Definition 7.6 The Euler vector field

$$
e_{t} \in \operatorname{Vect}\left(C F\left(\phi_{t}\right)\right), \quad e_{t}=\sum_{x \in \mathcal{I}(\phi)}(1-|x|) \partial_{x}
$$

Identify $C F\left(\phi_{t}\right) \cong C F\left(\phi_{t_{1}}\right)$ for all $t \in\left[t_{1}, t_{2}\right]$ and let

$$
E_{t_{1}}^{t_{2}}: C F\left(\phi_{t_{1}}\right) \rightarrow C F\left(\phi_{t_{2}}\right)
$$

denote the flow of the Euler vector field $e_{t}$ from time $t_{1}$ to time $t_{2}$. On generators we have

$$
E_{t_{1}}^{t_{2}}: x \mapsto \begin{cases}q^{\left(t_{2}-t_{1}\right)(1-|x|)} x & x \in \mathcal{I}^{\mathrm{c}}(\phi) \cup\left\{x^{\vee}, x^{\nabla}\right\} \\ q^{\int_{t_{1}}^{t_{2}}(1-\theta(x))} x & x \in \mathcal{I}^{\mathrm{si}}(\phi)\end{cases}
$$

Theorem 7.7 Let $\phi_{t}: L \rightarrow X$ be a Maslov flow of Lagrangian immersions with only transverse self-intersections and no triple intersections. Suppose that $t_{2}$ is sufficiently close to $t_{1}$ so that the bijection between the moduli spaces $\mathcal{M}\left(\phi_{t_{1}}\right)$ and $\mathcal{M}\left(\phi_{t_{2}}\right)$ is induced by a family of diffeomorphisms $\psi_{t_{1}}^{t_{2}}: X \rightarrow X$ mapping $\phi_{t_{1}}(L)$ to $\phi_{t_{2}}(L)$. Then the A-infinity structure maps $\mu_{d}^{t_{1}}$ for $\phi_{t_{1}}$ and $\mu_{d}^{t_{2}}$ for $\phi_{t_{2}}$ are related by

$$
\begin{aligned}
q^{\left(t_{2}-t_{1}\right)} E_{t_{1}}^{t_{2}}\left(\mu_{d}^{t_{1}}\left(x_{1}, \ldots, x_{d}\right)\right)=\mu_{d}^{t_{2}}\left(E_{t_{1}}^{t_{2}} x_{1}, \ldots, E_{t_{1}}^{t_{2}} x_{d}\right) & \\
\forall d & \geq 0, x_{1}, \ldots, x_{d} \in \mathcal{I}\left(\phi_{t_{1}}\right) \cong \mathcal{I}\left(\phi_{t_{2}}\right) .
\end{aligned}
$$


Proof Since $\mu_{d}$ is defined by counting holomorphic disks, we have for all $x_{k}$ 's selfintersection points

$$
\begin{aligned}
& \frac{d}{d t} q^{\left(t-t_{1}\right)}\left(E_{t_{1}}^{t}\right)^{-1} \mu_{d}^{t}\left(E_{t_{1}}^{t} x_{1}, \ldots, E_{t_{1}}^{t} x_{d}\right) \\
& =\frac{d}{d t} q^{t-t_{1}} q^{\int_{t_{1}}^{t}\left(1-\theta\left(x_{0}\right)\right)} \mu_{d}^{t}\left(q^{\int_{t_{1}}^{t}\left(1-\theta\left(x_{1}\right)\right)} x_{1}, \ldots, q^{\int_{t_{1}}^{t}\left(1-\theta\left(x_{d}\right)\right)} x_{d}\right) \\
& =\left(\sum_{k>0} \theta\left(x_{k}\right)-d+2-\theta\left(x_{0}\right)+\left(\theta\left(x_{0}\right)-1\right)-\sum_{k>0}\left(\theta\left(x_{k}\right)-1\right)\right) \\
& \ln (q)\left(E_{t_{1}}^{t}\right)^{-1} \mu_{d}^{t}\left(E_{t_{1}}^{t} x_{1}, \ldots, E_{t_{1}}^{t} x_{d}\right)=0
\end{aligned}
$$

by Lemma 7.4. The claim in the Lemma holds when $t=t_{1}$ since $E_{t_{1}}^{t_{1}}$ is the identity. By (56) the claim holds for all $t$. The general case (when some $x_{k} \in \mathcal{I}^{\mathrm{c}}\left(\phi_{t}\right)$ ) is similar.

Because of the signs in the Euler flow above, the flow may not preserve the space of Maurer-Cartan solutions which are required to have positive $q$-valuation. Let

$$
M C^{>E}\left(\phi_{t}\right)=\left\{b \in M C\left(\phi_{t}\right) \mid \operatorname{val}_{q}(b)>E\right\}
$$

be the subset of solutions to the weak Maurer-Cartan equation having $q$-valuation at least $E$.

Corollary 7.8 Let $\phi_{t}: L \rightarrow X$ be a Maslov flow of Lagrangian immersions with transverse self-intersections for $t \in\left[t_{1}, t_{2}\right]$. The Euler flow $E_{t_{1}}^{t_{2}}: C F\left(\phi_{t_{1}}\right) \rightarrow C F\left(\phi_{t_{2}}\right)$ (resp. its inverse) maps

$$
E_{t_{1}}^{t_{2}}: M C^{>E}\left(\phi_{t_{1}}\right) \rightarrow M C^{>E-(\operatorname{dim}(L)-1)\left(t_{2}-t_{1}\right)}\left(\phi_{t_{2}}\right)
$$

resp.

$$
M C^{>E}\left(\phi_{t_{2}}\right) \rightarrow M C^{>E-2\left(t_{2}-t_{1}\right)}\left(\phi_{t_{2}}\right) .
$$

The potentials are preserved up to an overall power of $q$, that is,

$$
\left(E_{t_{1}}^{t_{2}}\right)^{*} W_{t_{2}}=q^{\left(t_{2}-t_{1}\right)} W_{t_{1}}
$$

and the Euler flow lifts to an isomorphism

$$
H F\left(\phi_{t_{1}}, b_{t_{1}}\right) \rightarrow H F\left(\phi_{t_{2}}, b_{t_{2}}\right) .
$$

Proof We have

$$
\begin{aligned}
\mu_{0}^{t_{2}}(1) & +\mu_{1}^{t_{2}}\left(E_{t_{2}}^{t_{2}} b\right)+\mu_{2}^{t_{2}}\left(E_{t_{1}}^{t_{2}} b, E_{t_{1}}^{t_{2}} b\right)+\ldots \\
& =q^{\left(t_{2}-t_{1}\right)} E_{t_{1}}^{t_{2}} \mu_{0}^{t_{2}}(1)+q^{\left(t_{2}-t_{1}\right)} E_{t_{1}}^{t_{2}} \mu_{1}^{t_{2}}(b)+q^{\left(t_{2}-t_{1}\right)} E_{t_{1}}^{t_{2}} \mu_{2}^{t_{2}}(b, b)+\ldots \\
& =q^{\left(t_{2}-t_{1}\right)} E_{t_{1}}^{t_{2}}\left(\mu_{0}^{t_{2}}(1)+\mu_{1}^{t_{2}}(b)+\mu_{2}^{t_{2}}(b, b)+\ldots\right) .
\end{aligned}
$$




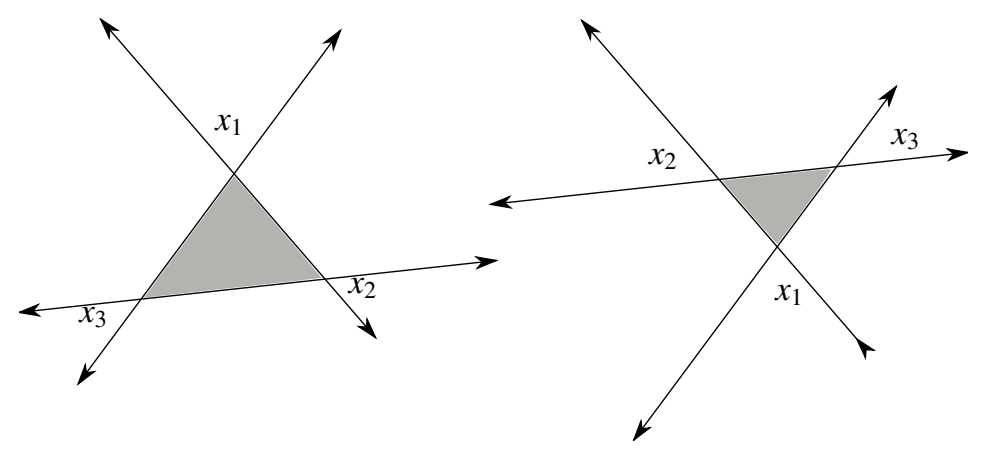

Figure 16: Behavior under a triple intersection

Since the real-grading on $C F\left(\phi_{t}\right)$ takes values between 0 and $n=\operatorname{dim}(L)$, the Euler flow $E_{t_{1}}^{t_{2}}: C F\left(\phi_{t_{1}}\right) \rightarrow C F\left(\phi_{t_{2}}\right)$ satisfies

$$
\frac{d}{d t} \operatorname{val}_{q}\left(\left(E_{t_{1}}^{t}\right)^{-1}(x)\right)=|x|-1 \in[-2, \operatorname{dim}(L)-1]
$$

for self-intersection points $x \in \mathcal{I}\left(\phi_{t}\right)$ by (54). Hence the forward flow maps $M C^{>E}\left(\phi_{t_{1}}\right)$ to $M C^{>E-\left(t_{2}-t_{1}\right)(1-\operatorname{dim}(L))}\left(\phi_{t_{2}}\right)$, and preserves the potentials up to an overall power of $q$. Similarly, the reverse Euler flow maps $M C^{>E}\left(\phi_{t_{2}}\right)$ to $M C^{>E-2\left(t_{2}-t_{1}\right)}\left(\phi_{t_{2}}\right)$, and preserves the potentials up to an overall power of $q$. Taking the directional derivative of $\mu_{0}^{b}(1)$ in the direction of a class $c$ implies that $H F\left(\phi_{t_{1}}, b_{t_{1}}\right)$ is mapped to $H F\left(\phi_{t_{2}}, b_{t_{2}}\right)$ isomorphically.

In order to complete the proof of Theorem 1.2, it remains to deal with triple intersections, that is, triples $x_{1}, x_{2}, x_{3} \in L$ with $\phi\left(x_{1}\right)=\phi\left(x_{2}\right)=\phi\left(x_{3}\right)$.

Lemma 7.9 (Triple intersection lemma)

(a) (Dimension greater than two) Suppose that $\operatorname{dim}(X) \geq 4$. Let $\phi_{0}: L \rightarrow X$ be a Lagrangian immersion without triple intersections and with only transverse selfintersections $L \times{ }_{\phi} L-\Delta_{L}$. Then for any $l \geq 2$, there exists an open $C^{l}$-dense set of Maslov flows $\phi_{t}: L \rightarrow X, t \in[0, T]$ between $\phi_{0}$ and $\phi_{T}$ for which there are no triple self-intersection points;

(b) (Dimension two) In dimension $\operatorname{dim}(X)=2$, immersed Lagrangian Floer theory $H F\left(\phi_{t}, b_{t}\right)$ is invariant under a Maslov flow $\phi_{t}: L \rightarrow X, t \in[0,2]$ that develops a triple intersection (but no quadruple intersections). 
Proof First we give the proof of (a), which uses a Sard-Smale argument and a dimension count. Suppose that $\operatorname{dim}(X) \geq 4$. Consider the universal space of triple points

$$
\begin{aligned}
& \mathcal{M}^{\text {univ,trip }}:=\left\{\begin{array}{l|l}
\left(t_{0}, \phi, H, x_{1}, x_{2}, x_{3}\right) & \begin{array}{l}
x_{1}, x_{2}, x_{3} \text { distinct } \\
\phi_{t_{0}}\left(x_{1}\right)=\phi_{t_{0}}\left(x_{2}\right)=\phi_{t_{0}}\left(x_{3}\right) \\
\left.\dot{\phi}_{t}=\phi_{t}^{*}\left(\mathrm{~d} H_{t}+\alpha_{t}-\alpha_{L}\right)\right\}
\end{array}
\end{array}\right\} \\
& \subset[0,1] \times \operatorname{Lag}(L, X)_{l} \times C^{l}([0,1] \times X) \times L^{3} .
\end{aligned}
$$

By assumption the self-intersections of $\phi_{0}$ are transverse. As in Theorem 2.6, Hamiltonian variations generated by $H_{t} \in C^{l}(X)$ together with $\left(D_{x} \phi\right)\left(T_{x} L\right)$ span the tangent space $T_{\phi(x)} L$ at any point $x \in L$. It follows that the locus (57) is cut out transversally. By the Sard-Smale theorem, regular values of the projection

$$
\pi: \mathcal{M}^{\text {univ,trip }} \rightarrow C^{l}([0,1] \times X)
$$

are comeager. For regular values $H$, the submanifold of triple intersections $\pi^{-1}(H)$ is of expected dimension -1 , hence empty.

The argument for (b) is rather different, since triple intersections cannot be avoided in families; instead we must examine the holomorphic triangles more carefully. Suppose that $\operatorname{dim}(X)=2$. For a triple intersection point of $\phi_{0}$, denote the intersection points $x_{1}, x_{2}, x_{3} \in L$ with $\phi_{0}\left(x_{1}\right)=\phi_{0}\left(x_{2}\right)=\phi_{0}\left(x_{3}\right)$. If a holomorphic polygon $u: S \rightarrow X$ with boundary in $\phi$ has only one of these points $x_{k}, k \in\{1,2,3\}$ as a vertex, then that $u$ extends in a continuous family $u_{t}$ with boundary in $\phi_{t}$ for all $t$ past the triple intersection time 0 . On the other hand, $b_{t} \in M C\left(\phi_{t}\right)$ for $t<0$ implies that if the coefficient of two or more $\bar{x}_{i}, \bar{x}_{j}, i, j \in\{1,2,3\}$ of the odd generators $\bar{x}_{1}, \bar{x}_{2}, \bar{x}_{3}$ in the element $b_{t} \in C F\left(\phi_{t}\right)$ are non-zero, then the triangle in Figure 16 implies that the Floer cohomology is obstructed. Indeed, if say the coefficients $b\left(\bar{x}_{1}\right), b\left(\bar{x}_{2}\right)$ are non-zero and the triangle has area $A$, smaller than the area of any other holomorphic polygon contributing to $\mu_{0}^{b}(1)$ then we would have

$$
\mu_{0}^{b}(1)=\mu_{2}(b, b)=b\left(\bar{x}_{1}\right) b\left(\bar{x}_{2}\right) q^{A} x_{3}+\text { higher order in } q .
$$

So at most one element of $x_{1}, x_{2}, x_{3}$ has non-zero coefficient in $b_{t}$. It follows that the disks $u_{t}: C \rightarrow X$ that meet two or more of the generators $x_{1}, x_{2}, x_{3}$ do not contribute to the maps $\mu_{d}^{b_{t}}(1)$.

\section{Curve shrinking and gluing at a tangency}

In this section we modify estimates of Ekholm-Etnyre-Sullivan [23] to show invariance

under the birth or death of a pair of self-intersection points. The Floer differential 
contains a term of small $q$-valuation that connects the two new generators. This makes the Floer cohomology invariant under the birth or death, while the modification of the curvature of the Fukaya algebra can be cancelled by a suitable weakly bounding cochain.

The main result of this section is a description of how the holomorphic disks change as the immersion passes through a self-tangency, and is a modification of the results of [23, Chapter 10] to the setting of treed disks. Let $\left(\phi_{t}: L \rightarrow X\right)_{t \in[-\epsilon, \epsilon]}$ be a family of Lagrangian immersions with a single value $t=0$ for which there is a birth of a pair of self-intersection points at $v \in \phi_{0}(L)$. Let

$$
v_{+}, \bar{v}_{+}, \bar{v}_{-}, v_{-} \in \mathcal{I}^{\mathrm{si}}\left(\phi_{\epsilon}\right)
$$

denote the additional generators in the Floer cochains for $\phi_{\epsilon}$ in relation to $\phi_{-\epsilon}$ depicted in the local model in Figure 11, where $\bar{v}_{+}, \bar{v}_{-}$are distinguished as the generators that are the incoming corners of the small strip in Figure 2; that is, with the curved branch $L_{2}$ in (34) ordered before the flat branch $L_{1}$ for the intersection with negative $q_{1}$ coordinate, and the reverse for the intersection with positive $q_{1}$ coordinate. We say that $\underline{x}_{t} \subset \mathcal{I}^{\text {si }}\left(\phi_{t}\right)$ is an admissible family of generators if and only if

$$
\phi\left(x_{i, t}\right) \rightarrow v, x_{i, t} \in \underline{x}_{t}, i>0 \text { resp. } i=0 \text { implies } x_{i, t} \notin\left\{v_{+}, v_{-}\right\} \text {resp. } x_{i, t} \in\left\{v_{+}, v_{-}\right\} ;
$$

that is, the generators $\bar{v}_{+}, \bar{v}_{-}$are excluded as outputs and $v_{+}, v_{-}$are excluded as inputs. In the following the reference to the Donaldson hypersurface $D$ may be dropped to simplify notation so that the moduli spaces of holomorphic treed disks will be denoted $\overline{\mathcal{M}}\left(\phi_{t}\right)$.

Theorem 8.1 Let $\phi_{t}: L \rightarrow X, t \in[-T, T]$ be an admissible family of Lagrangian immersions with a self-tangency at $t=0$ at $y \in X$. Suppose that admissible perturbations $\underline{P}=\left(P_{\Gamma}\right)$ have been chosen for $\phi_{0}$ using some Donaldson hypersurface $D$. Then the same hypersurface $D$ and collection $\underline{P}=\left(P_{\Gamma}\right)$ of perturbations are regular for adapted holomorphic disks with boundary in $\phi_{t}, t \in[-\epsilon, \epsilon]$ for $\epsilon$ sufficiently small and

(a) (Small strips, similar to Lemma 2.14 in [23]) There exists $\epsilon>0$ such that for any $t \in(0, \epsilon)$ there exist holomorphic strips of index one

$$
\left[u_{+}(t): C \rightarrow X\right] \in \mathcal{M}\left(\phi_{t}, \bar{v}_{+}, v_{-}\right), \quad\left[u_{-}(t): C \rightarrow X\right] \in \mathcal{M}\left(\phi_{t}, \bar{v}_{-}, v_{+}\right),
$$

connecting $\bar{v}_{+}$to $v_{-}$resp. $\bar{v}_{-}$to $v_{+}$with area $A\left(u_{ \pm}(t)\right) \rightarrow 0$ as $t \rightarrow 0$, as in Figure 2, and any strip $u: C \rightarrow X$ with boundary in $\phi_{t}$ between $\bar{v}_{+}$and $v_{-}$of sufficiently small energy $E(u)$ is equal to $u_{+}(t)$ or $u_{-}(t)$. 

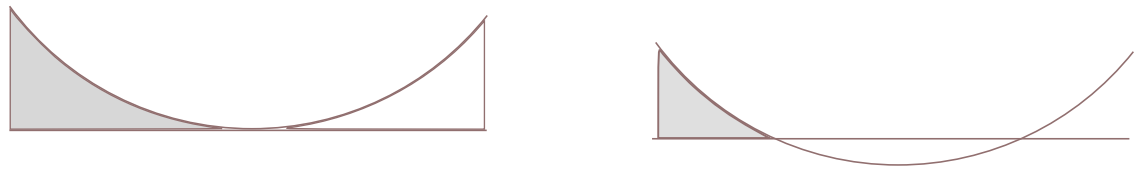

Figure 17: Shrinking a holomorphic curve at a tangency
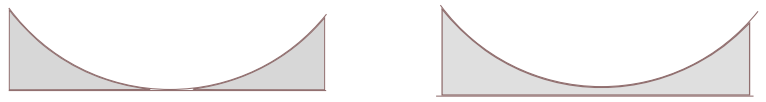

Figure 18: Gluing a holomorphic curve at a tangency

(b) (Curve shrinking, similar to Proposition 2.16 in [23]) Let $\underline{x}_{s} \in \mathcal{I}\left(\phi_{s}\right)$ for $s \in$ $[0, \delta]$ be a admissible family of self-intersection points/critical points. There exist $\varkappa>0$ such that for any $s \in(-1 / \varkappa, 0)$ there exists an orientationpreserving bijection

$$
G_{s}: \mathcal{M}_{\Gamma_{0}}\left(\phi_{0}, \underline{x}_{0}\right)_{\rho} \rightarrow U_{s} \subset \mathcal{M}_{\Gamma_{s}}\left(\phi_{s}, \underline{x}_{s}\right)_{\rho}-\left\{u_{ \pm}\right\}
$$

where $u_{ \pm}$are the strips from the previous item, $U_{s}$ is a $C^{0}$ neighborhood of $\mathcal{M}_{\Gamma_{0}}\left(\phi_{0}, \underline{x}_{0}\right)_{\rho}$ in $\mathcal{M}_{\Gamma_{s}}\left(\phi_{s}, \underline{x}_{s}\right)_{\rho}$, with the property that $\lim _{s \rightarrow 0} G_{s}(u)=u$. See Figure 17.

(c) (Gluing at a tangency, similar to Proposition 2.17 in [23]) Let $\Gamma_{0}$ be a combinatorial type of tree disk $E_{0} \subset \operatorname{Edge}(\Gamma)$ a subset of the finite edges corresponding to nodes $q_{e} \subset C$ mapping to the self-tangency point $x$, and no semi-infinite edges $T_{e} \subset T$ map to the self-tangency $x$. Let $\Gamma_{t}$ denote the combinatorial type obtained by collapsing the edges $E_{0}$. Suppose that the almost complex structure $J$ is in standard form near the self-tangency. Then there exists $\varkappa>0$ such that for $s \in(0,1 / \varkappa)$ there exists a bijection

$$
G_{s}: \mathcal{M}_{\Gamma_{0}}\left(\phi_{0}\right)_{\rho} \rightarrow U_{s} \subset \mathcal{M}_{\Gamma_{s}}\left(\phi_{s}\right)_{\rho}
$$

where $U_{s}$ is a Gromov neighborhood of $\mathcal{M}_{\Gamma_{0}}\left(\phi_{0}, \underline{x}_{0}\right)$ in $\mathcal{M}_{\Gamma_{s}}\left(\phi_{s}, \underline{x}_{s}\right)_{\rho}$, with the property that $\lim _{s \rightarrow 0} G_{s}(u)=u$. See Figure 18. After multiplying the given orientations by the sign (47) in the definition of the structure maps the map $G_{s}$ is orientation preserving. Furthermore, given any sequence $u_{\nu} \in \mathcal{M}_{\Gamma_{s}}\left(\phi_{s}\right)_{\rho}$ there exists a subsequence converging to some $u \in \mathcal{M}_{\Gamma_{0}}\left(\phi_{0}\right)_{\rho}$.

Proof of Theorem 8.1 (a) The proof is essentially that of Lemma 2.14 in [23], but we reproduce a proof for completeness. See also [40] for similar gluing estimates. 
Note first that the Lagrangians in the local model (14) are products. Hence any holomorphic curve of $u: C \rightarrow X$ may be written in a neighborhood of $y$ as a product of maps $u_{1}: C \rightarrow X$ and $u_{2}: C \rightarrow X$ with boundary in $L_{1}^{\prime}, L_{1}^{\prime \prime}$ and $L_{2}(s)^{\prime}, L_{2}(s)^{\prime \prime}$. Let $u_{1}: \mathbb{R} \times[0,1] \rightarrow \mathbb{C}$ denote the holomorphic strip with boundary on $L_{1}^{\prime}, L_{2}(s)^{\prime \prime}$ whose image is the region

$$
x_{1}^{2}+\left(y_{1}-1 \pm t\right) \in\left[0, \sqrt{1-x_{1}^{2}}\right] .
$$

This is the shaded region in Figure 2, which exists by the Riemann Mapping Theorem, see [58]. Let $u_{2}, \ldots, u_{n}$ be the constant maps in the other components. Then $u=$ $\left(u_{1}, \ldots, u_{n}\right)$ is the desired map.

We claim that there are no other maps of equally small energy. By energy quantization Lemma 5.4 there exists $\hbar$ so that any non-trivial holomorphic polygon mapping a corner to an element $x \in \mathcal{I}\left(\phi_{t}\right)$ not equal to $y$ has energy at least $\hbar$. Fix $\epsilon$ sufficiently small so that $A(u(\epsilon))<\hbar / 2$. Any other map $u^{\prime}: C^{\prime} \rightarrow X$ of area at most, say, $2 A(u(\epsilon))$ must be such that each component of the surface part $u_{S^{\prime}}^{\prime}$ meets the given neighborhood of $y$. The surface part $u_{S^{\prime}}^{\prime}$ is contained in a small neighborhood of the intersection point, by a diameter estimate involving the mean value inequality explained in Sikorav [51, 4.4.1] (for the closed case; the case with Lagrangian boundary is the same). Then the components $u_{2}^{\prime}, \ldots, u_{n}^{\prime}$ must be constant by the maximum principle, while the first component is, up to translation, the map $u$ given above up to an automorphism of $\mathbb{R} \times[0,1]$.

The proofs of the shrinking and gluing parts Theorem 8.1 are generalizations of results of Ekholm-Etnyre-Sullivan [23] that rely on a local model for the tangency. We prove parts (b) and (c) of Theorem 8.1 in the remainder of this section. As for similar gluing results for pseudoholomorphic curves, the results depend on a quantitative version of the implicit function theorem used by Floer to prove gluing of trajectories:

Lemma 8.2 (Floer's Picard Lemma, [27, Proposition 24]) Let $f: V_{1} \rightarrow V_{2}$ be a smooth map between Banach spaces that admits a Taylor expansion $f(v)=f(0)+$ $d f(0) v+N(v)$ where $d f(0): V_{1} \rightarrow V_{2}$ is Fredholm and has a right inverse $G: V_{2} \rightarrow V_{1}$ satisfying the uniform bound

$$
\|G N(u)-G N(v)\| \leq C(\|u\|+\|v\|)\|u-v\|
$$

for some constant $C$. Let $B(0, \epsilon)$ denote the $\epsilon$-ball centered at $0 \in V_{1}$ and assume that

$$
\|G f(0)\| \leq \frac{1}{8 C} .
$$

Then for $\epsilon<\frac{1}{4 C}$, the zero-set of $f^{-1}(0) \cap B(0, \epsilon)$ is a smooth submanifold of dimension $\operatorname{dim}(\operatorname{Ker}(d f(0)))$ diffeomorphic to the $\epsilon-$ ball in $\operatorname{Ker}(d f(0))$. 
We review the Sobolev spaces and estimates from Etnyre-Ekholm-Sullivan [23] necessary to prove shrinking. Given a curve $u: C \rightarrow X$ with boundary in $\partial u: \partial C \rightarrow L$. In the local model of a self-tangency move (14) $u$ is a product of components $u_{1}, \ldots, u_{n}$ to $\mathbb{C}$ with the given Lagrangian boundary conditions. By the local model, we may assume that $L$ is locally the union $\phi(L) \cap U \cong L_{1} \cup L_{2}$ of components $L_{1}, L_{2}$ with $L_{1} \subset \mathbb{C}^{n}$ linear and $L_{2} \subset \mathbb{C}^{n}$ the product of a part of a circle of radius one with a linear space, whose center will move down as one performs the isotopy. Up to rescaling this is a mean curvature flow.

Definition 8.3 (Pre-shrinking) We first define a simplified family of immersions developing a self-tangency. Let $U$ be a ball of radius $\left(R+\frac{1}{2} R^{a}\right)^{-1}$ around the self-tangency point $v$, the origin in local coordinates, where $a \in(0,1)$ is close to 1 . Let $b_{R} \in \mathbb{C}^{\infty}(\mathbb{R})$ be a non-decreasing function with support in $\left[0, R^{-1}\right)$ and

$$
b_{R}(r)=\left(R+R^{a}\right)^{-2}, \quad \forall r \in\left[0,\left(R+\frac{1}{2} R^{a}\right)^{-1}\right)
$$

and

$$
\sup \left|D^{k} b_{R}\right| \leq O\left(R^{2 k-(2 k+1) a}\right), k \leq 3
$$

Let

$$
h_{R}: \mathbb{C}^{n} \rightarrow \mathbb{R}, \quad z \mapsto \operatorname{Re}\left(z_{1}\right) b_{R}(|z|) .
$$

For $s>0$ let

$$
\Psi_{R}^{s}: X \rightarrow X
$$

denote the time $s$ Hamiltonian flow of $h_{R}$. Thus $\Psi_{R}^{s}$ is a translation by $\left(R+R^{a}\right)^{-2} s$ in a small ball of radius $\left(R+\frac{1}{2} R^{a}\right)^{-1}$ around the origin, and the identity outside a ball of radius $R^{-1}$. Let

$$
L_{2}^{R}(s)=\Psi_{R}^{s}\left(L_{2}\right)
$$

and let

$$
\phi_{s}^{\text {loc }}: L \rightarrow X
$$

denote the immersion obtained by patching together $\phi$ outside of the ball around $x$ with the immersions with images $L_{1}, L_{2}^{R}(s)$. The family $\phi_{s}^{\text {loc }}$ is a Maslov flow up to rescaling locally, by Example 2.4, since the change in radius in the circle is equivalent up to dilation centered at a point on the branch $L_{1}$ to a translation.

We now define an approximately holomorphic disk ending at one of the self-intersection points near the tangency. Let $\beta:[0,1] \rightarrow[0,1]$ be a smooth surjective map which is 
constant in a small neighborhood of $\{0,1\}$. Let $u: C \rightarrow X$ be a treed holomorphic disk and $u_{R}$ the deformation of $u$ in a neighborhood of the self-tangency given by

$$
u_{R}: \mathbb{R} \times[0,1] \rightarrow X, \quad u_{R}(\tau+i t)=\Psi_{R}^{\beta(t)}(u(\tau+i t))
$$

so that $u_{R}$ has the deformed Lagrangian boundary conditions.

The pre-shrunk map is defined by cutting off the variation of the local model of the previous paragraph near the intersection point. Let $\varkappa=\varkappa(R)$ be such that the intersection points of $L_{1}$ and $L_{2}^{R(\varkappa)}(s)$ are

$$
L_{1} \cap L_{2}^{R(\varkappa)}(s)=\left\{ \pm\left(\left(\varkappa+\varkappa^{a}\right)^{-1}, 0, \ldots, 0\right)\right\} .
$$

Choose a family of metrics $g(R, s, t)$ such that the Lagrangian $L_{1}$ is totally geodesic for $t=0$ and $L_{2}^{R(\varkappa)}$ is totally geodesic for $t=1$. Locally near each node $q_{k}$ mapping to the self-tangency the map $u_{R}$ may be written as the geodesic exponential of a section of the tangent bundle

$$
\xi_{R, k}: E_{p_{k}}(-\varkappa) \rightarrow \mathbb{C}^{n}, \quad \exp ^{R, t}\left(\xi_{R, k}(\tau+i t)\right)=u_{R}(\tau+i t), \quad \forall j .
$$

Define a treed disk $w_{\varkappa}: C \rightarrow X$ by patching as follows:

$$
w_{\varkappa}(\zeta)=\left\{\begin{array}{ll}
u(\zeta) & \zeta \in C \backslash \cup_{k} E_{k}(\varkappa) \\
\exp ^{R, t}\left(\xi_{R, k}(\zeta)\right) & \zeta \in E_{k}(\varkappa) \\
v(\zeta) & \zeta \in \cup_{k} E_{k}\left(-\varkappa-\varkappa^{a}\right)
\end{array} .\right.
$$

This ends the definition.

We claim that there is a unique holomorphic treed disk near the approximatelyholomorphic disk defined in the previous paragraph. Since the almost complex structure $J_{\Gamma}$ in a neighborhood $U$ of the self-tangency $v$ is assumed to be standard $\left.J_{\Gamma}\right|_{U}=J_{\mathbb{C}^{n}}$, and so invariant under rescaling, it will suffice to prove Theorem 8.1 (b) for the family $\phi_{s}^{\text {loc }}$. The Sobolev spaces used for shrinking are defined using a weight function constant on the ends.

Definition 8.4 (Weighted Sobolev spaces) Let $\lambda>0$ be a small weight and $e_{\varkappa}: C \rightarrow$ $\mathbb{R}$ a function with

$$
e_{\varkappa}(\tau+i t)=\left\{\begin{array}{ll}
e^{-\lambda \tau} & \tau \in(0, \varkappa) \\
e^{-\lambda \varkappa} & \tau>\varkappa
\end{array} .\right.
$$

Let $\mathcal{B}_{2,-\lambda, \varkappa}$ denote the Banach manifold of treed disks $u: C \rightarrow X, \partial u: \partial C \rightarrow L$ bounding $\phi$ such that on each component, $u$ and $\partial u$ are of finite $e_{\varkappa}$-weighted $W^{2,2}$ norm as in the proof of Proposition 3.2. Let $\mathcal{E}_{1,-\lambda, \varkappa}$ be the corresponding bundle of one-forms as in (21). 
The following are the necessary ingredients in the Picard lemma:

Proposition 8.5 (Zeroth, first, and second order estimates for shrinking)

(a) There exists a constant $C_{0}>0$ such that for all $\lambda>0$ sufficiently small and $\varkappa>0$ sufficiently large, the pre-glued section $w_{\varkappa}$ defined above has norm

$$
\left\|\mathcal{F}_{w_{\varkappa}}(0)\right\|_{1,-\lambda, \varkappa} \leq C_{0} e^{-\lambda \varkappa} \varkappa^{-1-a / 2} .
$$

(b) There exists a constant $C_{1}>0$ such that the linearized operator at the pre-glued solution satisfies the uniform estimate

$$
\|\xi\|_{2,-\lambda, \varkappa} \leq C_{1} \varkappa^{1+\delta}\left\|\tilde{D}_{w_{\varkappa}} \xi\right\|_{1,-\lambda, \varkappa} .
$$

(c) There exists a constant $C_{2}>0$ such that the non-linear term in the map $\mathcal{F}_{w_{\varkappa}}$ satisfies

$$
\left\|N\left(\xi_{1}\right)-N\left(\xi_{2},\right)\right\|_{1,-\lambda, \varkappa} \leq C_{2} e^{\lambda \varkappa}\left(\left\|\xi_{1}\right\|_{2,-\lambda, \varkappa}+\left\|\xi_{2}\right\|_{2,-\lambda, \varkappa}\right)\left\|\xi_{1}-\xi_{2}\right\|_{2,-\lambda, \varkappa}
$$

Sketch of proof We sketch the arguments, without going into full detail as in EkholmEtnyre-Sullivan [23, Chapter 8]. The exponential decay factor in the zero-th order estimate (a) arises from the choice of Sobolev weighting function and the second factor arises from the cutoff used to define the map $w_{\varkappa}$, see [23, Lemma 8.10]. The first order estimate is similar to [23, Lemma 8.11]. Suppose otherwise so that there exists a sequence $\xi_{\nu}, \nu \in \mathbb{Z}_{>0}$ with norm one such that the right-hand-side of (60) approaches zero for some sequence of gluing parameters $\varkappa_{\nu}$. Let $\alpha_{1}: C \rightarrow \mathbb{R}$ be a cutoff function equal to 0 in a neighborhood of the markings mapping to the self-intersection and with first and second derivatives of order $\varkappa^{-a}$; then

$$
\left\|\alpha_{1} \xi_{\nu}\right\|_{2,-\lambda, \varkappa} \leq C\left\|\left(\bar{\partial} \alpha_{1}\right) \xi_{\nu}\right\|_{1,-\lambda, \varkappa}+\left\|\alpha \tilde{D}_{w_{\varkappa}} \xi_{\nu}\right\|_{1,-\lambda, \varkappa}
$$

The first term is order $\varkappa^{-a}$ because of the estimates on the cutoff function while the second is order $\varkappa^{-1-\delta}$ by assumption on $\xi_{\nu}$. On the other hand, let $\alpha_{2}: C \rightarrow \mathbb{R}$ be a cutoff function equal to 1 on the strip-like ends, with first and second derivatives of order $\varkappa^{-a}$. Let $\hat{\varphi}:\left(-\infty,-\varkappa+\varkappa^{a}\right) \rightarrow \mathbb{R}$ be the function that equals the difference of angles $\theta(\varkappa)-\theta(\tau)$ between the tangent line $L_{2}^{\varkappa}(1)$ and the real line. The function $\hat{\varphi}$ extends to a cutoff function

$$
\varphi:\left(-\infty,-\varkappa+\varkappa^{a}\right) \times[0,1] \rightarrow \mathbb{C}
$$

with

$$
\begin{aligned}
0 & =\varphi(\tau+i t), & & (\tau, t) \in\left(-\infty,-\varkappa+\varkappa^{a}\right) \times\{0\} \\
\hat{\varphi}(\tau) & =\varphi(\tau+i t), & & (\tau, t) \in\left(-\infty,-\varkappa+\varkappa^{a}\right) \times\{1\}
\end{aligned}
$$


with the same derivative estimates. Let

$$
\mu=\operatorname{diag}(\varphi, 1, \ldots, 1) .
$$

Then we have

$$
\begin{aligned}
\left\|\alpha_{2} \mu \xi_{\nu}\right\|_{2,-\lambda, \varkappa_{\nu}} & \leq C\left\|e^{-\lambda \varkappa_{\nu}} \alpha_{2} \mu \xi_{\nu}\right\|_{2, \varkappa_{\nu}} \\
& \leq C \varkappa\left(\left\|e^{-\lambda \varkappa_{\nu}}\left(\bar{\partial} \alpha_{2} \mu\right) \xi_{\nu}\right\|_{1, \varkappa_{\nu}}+\left\|e^{-\lambda \varkappa_{\nu}} \alpha_{2, \varkappa_{\nu}} \mu \tilde{D}_{u} \xi_{\nu}\right\|_{1, \varkappa_{\nu}}\right.
\end{aligned}
$$

The first coefficient of $\xi_{\nu}$ arises from the fixed angle $\theta\left(\varkappa_{\nu}\right)$ which is approximately $1 / \varkappa_{\nu}$ for $\varkappa_{\nu}$ large. Thus we obtain that $\left\|\xi_{\nu}\right\|_{2,-\lambda, \varkappa_{\nu}}$ converges to zero as $\nu \rightarrow \infty$ which is a contradiction. Finally the estimate for the non-linear term is similar to [23, Lemma 8.17] and follows from

$$
\sup _{z \in C}|\xi(z)| \leq e^{\lambda \varkappa}\|\xi\|_{2,-\lambda, \varkappa}
$$

Floer's version of the Picard Lemma 8.2 and Proposition 8.5 produce the desired family of holomorphic treed disks with Lagrangian boundary condition in $\phi_{s}^{\text {loc }}$. The map $\phi_{s}^{\text {loc }}$ is related to $\phi_{s}$ by an isotopy of self-transverse Lagrangians of $X$, and so $\phi_{s}^{\text {loc }}$ and $\phi_{s}$ are related by a family of diffeomorphisms of $X$ which approaches the identity as $\varkappa \rightarrow \infty$. As in Corollary 3.3, in such a setting the spaces of holomorphic curves are in bijection. This proves the gluing part in (b) of Theorem 8.1, that is, the existence of the map. The Gromov convergence

$$
\lim _{s \rightarrow 0} G_{s}(u)=u
$$

follows since $E\left(G_{s}(u)\right) \rightarrow E(u)$ as $s \rightarrow 0$ and each component of the limit is obtained by a suitable rescaling sequence, by construction.

The existence of an inverse (that is, surjectivity of the above construction onto the space of nearby solutions) follows from the exponential decay estimates in Lemma 5.1 (a). We break the region bounded by $\operatorname{Re}\left(u_{s, 1}\right) \in\{1,2 / R\}$ the branches $L_{1}, L_{2}(s)$ into regions $\Omega_{s}, \Omega_{s}^{+}, \Omega_{s}^{++}$. On the region $\Omega_{s}^{++}$on which $\operatorname{Re}\left(u_{s, 1}\right) \geq 1$ we have uniform convergence in all derivatives. So

$$
u_{s}=\exp _{w_{s}}(\xi(s)), \text { for some } \xi(s), \quad\|\xi(s)\|<C \exp (-\lambda \varkappa) .
$$

On the region $\Omega_{s}^{+} \cong[-T(s), T(s)] \times[0,1]$ we write $u_{s, 1}$ in terms of its Fourier coefficients

$$
\begin{aligned}
\Theta_{s} \circ u_{s, 1}(\tau+i t)=\left(\tau-\tau_{0}(s)\right)+i t & +\sum_{n>0} c_{n}(s) \exp (2 \pi n(\tau-T(s))+i t) \\
& +\sum_{n<0} c_{n}(s) \exp (2 \pi n(\tau+T(s))+i t) .
\end{aligned}
$$


Uniform convergence at $\operatorname{Re}\left(u_{s, 1}\right)=1$ implies the Fourier constants $c_{n}(s), n>0$ converge to the Fourier constants $c_{n}(0)$ of $u_{0,1}$ as $s \rightarrow 0$, while the Fourier coefficients $c_{n}(s), n<0$ converge to zero by exponential decay of $u_{s, 1}$. It follows that

$\sup \left|\left(u_{s, 1}-w_{s, 1}\right)(\tau+i t)\right|^{p}+\left|\left(\mathrm{d} u_{s, 1}-\mathrm{d} w_{s, 1}\right)(\tau+i t)\right|^{p} \leq C \exp (-p|\tau-T(s)|) \quad$ on $\Omega_{s}^{+}$.

Thus for any $\epsilon$, there exists $s_{0}>0$ such that for $s<s_{0}$,

$$
\left\|\left.\left(u_{s}-w_{s}\right)\right|_{\Omega+_{s}}\right\|_{1, p,-\lambda} \leq \epsilon \exp (-\varkappa \lambda) .
$$

Finally the restriction of $u_{s, 1}-w_{s, 1} \mid \Omega_{s}$ to $\Omega_{s} \cong[-T(s), T(s)] \times[0,1]$ converges to zero uniformly, as well as its derivative. Thus for any $\epsilon>0$, there exists $s_{0}>0$ such that if $s<s_{0}$ then

$$
\sup \left|\left(u_{s, 1}-w_{s, 1}\right)(\tau+i t)\right|^{p}+\left|\left(\mathrm{d} u_{s, 1}-\mathrm{d} w_{s, 1}\right)(\tau+i t)\right|^{p} \leq \epsilon \exp (-\varkappa \lambda) .
$$

It follows that

$$
\left\|u_{S}-\left.w_{s}\right|_{\Omega_{s}}\right\|_{1, p,-\lambda} \leq \epsilon \exp (-\varkappa \lambda) .
$$

Putting everything together we find that for any $\epsilon>0$, there exists $s_{0}>0$ such that if $s<s_{0}$ then

$$
u_{s}=\exp _{w_{s}}(\xi(s)) \text {, for some } \xi(s) \text { with }\|\xi(s)\|_{1, p, \lambda} \leq \epsilon \exp (-\varkappa \lambda) .
$$

Since this quantity is less than the quantity $1 / 4 C$ in Floer's Picard Lemma 8.2, for $\epsilon$ sufficiently small, the map $u_{s}$ is the solution given by the Picard iteration.

The gluing at a tangency part (c) of Theorem 8.1 follows from a similar application of Floer's Picard Lemma 8.2. Similar to the set-up for shrinking, for $R \gg 0$ let $a_{R}:[0, \infty] \rightarrow \mathbb{R}$ be a smooth non-increasing function with support in $\left[0, R^{-1} / 2\right)$ with the properties

$$
a_{R}(r)=1 / R, \quad r \in\left[0,1 / R^{2}\right] ; \quad \sup _{r}\left|D_{r} a_{R}\right|=O(1) ; \quad \sup _{r}\left|D_{r}^{2} a_{R}\right|=O(R) .
$$

Let

$$
h_{R}: \mathbb{C}^{n} \rightarrow \mathbb{R}, \quad h_{R}(z)=\operatorname{Re}\left(z_{1}\right) a_{R}\left(\left|z_{1}\right|\right) .
$$

For $s>0$ let $\Phi_{R}^{s}$ denote the time $s$ Hamiltonian flow of $h_{r}$. Let $L_{2}^{R}(s)$ denote the Lagrangian submanifold obtained by applying $\Phi_{R}^{s}$ to $L_{2}$, and let $\gamma^{1}(s), \gamma_{R}^{2}(s)$ be paths tracing out $L_{1}$ and $L_{2}^{R}(s)$. Let $\phi_{s}^{\text {loc }}: L \rightarrow X$ be the family of immersions obtained by gluing in the local family to the immersion $\phi: L \rightarrow X$. Let $u: C \rightarrow X$ be a rigid holomorphic tree disk with boundary on $\phi$ and $I \subset\{0, \ldots, n\}$ such that the points $z_{i}, i \in I$ on the boundaries of the disk map to the self-intersection points. Let $s=(K \varkappa)^{-1}$ as below or in [23, Lemma 10.18] and let $\Xi_{\varkappa}$ denote the neck region bounded by the curves $\gamma_{R}^{2}(s), \gamma^{1}(s)$ and $u^{i \pm(k)}(\varkappa+i t)$ for $t \in[0,1]$. By the Riemann 
mapping theorem for a unique $A(\varkappa)$ there exists a biholomorphic map from the interior of rectangle

$$
\varphi_{\varkappa}:[-A(\varkappa), A(\varkappa)] \times[0,1]=: \Omega_{\varkappa} \rightarrow \mathbb{C}
$$

with a continuous extension with the properties

$$
\begin{aligned}
\varphi_{\varkappa}([-A(\varkappa), A(\varkappa)] \times\{0\}) & \subset \operatorname{Im}\left(\gamma_{1}\right) \\
\varphi_{\varkappa}([-A(\varkappa), A(\varkappa)] \times\{1\}) & \subset \operatorname{Im}\left(\gamma_{2}(s)\right) \\
\varphi_{\varkappa}(0+i[0,1]) & \subset\left\{\operatorname{Re}\left(z_{1}\right)=0\right\} .
\end{aligned}
$$

The pre-glued map is defined as follows. Let $u_{i_{ \pm}(k)}^{1}$ denote the first components of the maps $u_{i_{ \pm}(k)}$ meeting the tangency at nodes $q_{k}$, contained in components $C_{i_{ \pm}(k)}$. By gluing $C_{i_{ \pm}(k)}-E_{q_{k}}\left[-((1+a) \varkappa]\right.$ with $\Omega_{\varkappa}$ one obtains a treed holomorphic disk on which the maps $u_{k, \pm}^{1}$ glue together in a neighborhood of the node $q_{k}$. Let $u_{k, \pm}^{\prime}$ denote the remaining components of $u$ in the local model. Using the standard metric on $\mathbb{C}^{n-1}$ define maps $u_{k, \pm}^{\prime}$ to $\mathbb{C}^{n-1}$ by

$$
u_{k, \pm}^{\prime}(\tau+i t)=\exp \left(\xi_{k, \pm}^{\prime}(\tau+i t)\right)
$$

where in this setting the geodesic exponentiation exp is the identity map since the metric is constant. Let $E_{q_{k}, \pm}$ denote the image of the local coordinate near $q_{k}$ on the component $C_{i_{ \pm}(k)}$ adjacent to the node $q_{k}$. Pick a cutoff function $\alpha$ equal to 1 on $C-\cup_{k, \pm} E_{q_{k}, \pm}[-\varkappa+5]$ with support in the complement of $\cup_{k, \pm} E_{q_{k}, \pm}[-\varkappa+3]$, with derivatives uniformly bounded for $\varkappa \gg 0$. Define $w_{\varkappa}^{\prime}: C_{\varkappa} \rightarrow \mathbb{C}^{n-1}$ by

$$
w_{\varkappa}^{\prime}(\zeta)= \begin{cases}u_{k, \pm}^{\prime}(\zeta) & \zeta \in C_{i_{ \pm}(k)}-E_{q_{k}, \pm}[-\varkappa+5] \\ \exp _{0}^{t}\left(\alpha(\zeta) \xi_{ \pm, j}(\zeta)\right) & E_{q_{k}, \pm}[-\varkappa+5]-E_{q_{k}, \pm}[-\varkappa] \\ 0 & \zeta \in \Omega_{\varkappa} .\end{cases}
$$

Combining the components we define $w_{\varkappa}=\left(w_{\varkappa}^{1}, w_{\varkappa}^{\prime}\right)$.

A treed holomorphic disk nearby the preglued solution above is found using the Picard lemma. For a function $f: C_{\varkappa} \rightarrow \mathbb{R}$ and for each component $C_{i_{ \pm}(k)}$ adjacent to a node $q_{k}$ mapping to the self-tangency we denote by $f_{i_{ \pm}(k)}$ the restriction of $f$ to $C_{i_{ \pm}(k)}-E_{k}[-(1+a) \varkappa]$. Let $f_{k}$ denote the restriction to the region $\Omega_{k}(\varkappa)$ near the node $q_{k}$. For $\lambda>0$ let $e_{\lambda}^{i_{ \pm}(k)}$ denote the weight function on $C_{i_{ \pm}(k)}$ which equals 1 on $C_{i \pm(k)}-\cup E_{q_{k}}$ and equals $e^{\lambda|\tau|}$ in $E_{q_{k}}$. Let $\|\cdot\|_{k, \lambda, i}$ denote the Sobolev norm on distributions with $k$ square-integrable derivatives obtained from the weight function $e_{\lambda}^{i_{ \pm}(k)}$. Let $e_{\lambda}^{j}$ denote the weight function which equals $e^{\lambda|\tau|}$ on $\Omega_{k}$ and $\|\cdot\|_{k, \lambda, j}$ the resulting Sobolev norm. Define

$$
\|f\|_{k, \lambda, \varkappa}=\sum_{i \neq i_{ \pm}(k)}\left\|f_{i}\right\|_{k, 2}+\sum_{j}\left\|f_{i}\right\|_{k, \lambda, i_{ \pm}(k)}+\sum_{j}\left\|f_{j}\right\|_{k, \lambda, j}
$$


Let

$$
\mathcal{F}_{u}: T_{u} \mathcal{B}_{\Gamma} \rightarrow \mathcal{E}_{\Gamma, u}
$$

denote the map from (39) obtained from the given local trivializations of $\mathcal{B}_{\Gamma}$ and $\mathcal{E}_{\Gamma}$ near $u$. Let $\theta \gg \lambda$ be the smallest non-zero Kähler angle of the intersection where the angles are described in (53); see also [23, Lemma 8.13].

Proposition 8.6 (Zeroth, first, and second order estimates for gluing)

(a) There exists $C_{0}>0$ such that for all $\varkappa>0$ sufficiently large the pre-glued solution $w_{\varkappa}$ satisfies

$$
\left\|\mathcal{F}_{w_{\varkappa}}(0)\right\|_{1, \lambda, \varkappa} \leq C_{0} e^{(-\theta+2 K \lambda) \varkappa}
$$

(b) There exists $C_{1}$ such that for all $\varkappa$ sufficiently large the linearization $\tilde{D}_{w_{\varkappa}}$ of $\mathcal{F}_{w_{\varkappa}}$ satisfies [23, Equation (8.74), (8.90)]

$$
\|\xi\|_{2, \lambda, \varkappa} \leq C_{1}\left\|\tilde{D}_{w_{\varkappa}} \xi\right\|_{1, \lambda, \varkappa} .
$$

(c) There exists $C_{2}>0$ such that for all $\varkappa$ sufficiently large the non-linear part of the map $\mathcal{F}_{w_{\varkappa}}$ satisfies a uniform estimate [23, Lemma 8.18]

$$
\left\|N\left(\xi_{1}\right)-N\left(\xi_{2}\right)\right\|_{1, \lambda, \varkappa} \leq C_{2}\left(\left\|\xi_{1}\right\|_{2, \lambda, \varkappa}+\left\|\xi_{2}\right\|_{2, \lambda, \varkappa}\right)\left\|\xi_{1}-\xi_{2}\right\|_{2, \lambda, \varkappa}
$$

Proof The first is similar to [23, Lemma 8.13], the second similar to [23, Lemma 8.15], and the third to [23, Lemma 8.16]. All estimates are local and so apply to holomorphic treed disks with almost complex structure standard in a neighborhood of the tangency.

Floer's version of the Picard Lemma 8.2 and Proposition 8.6 produce the claimed family of holomorphic treed disks $u_{s}: C_{s} \rightarrow X$ with boundary in $\phi_{s}^{\text {loc }}$ converging to $u$ as $\varkappa \rightarrow 0$. As before, $\phi_{s}^{\text {loc }}$ and $\phi_{s}$ are related by a family of diffeomorphisms of $X$ which approaches the identity in all derivatives as $s \rightarrow 0$. The existence of an inverse to $G_{s}$ (that is, "surjectivity of gluing") follows from exponential decay for pseudoholomorphic curves of small energy. Suppose that $u_{s}$ Gromov converges to $u$; we must show that for $s$ sufficiently small the solution $u_{s}$ is the one produced by the Picard iteration, up to equivalence. Because the almost complex structure is constant and split near the self-tangency, it suffices to consider each component $u_{s, i}, i=1, \ldots, n$ of $u_{s}$ separately. For the components $u_{s, i}$ with $i \neq 1$, the Lagrangian boundary conditions are transverse and the Lagrangian version of the annulus lemma (see for example, Frauenfelder-Zemisch [30, Lemma 3.1]) implies that for $s$ sufficiently large, $u_{s, i}$ is arbitrarily close to the approximate solution $w_{s, i}$ in the Sobolev norm defined by 
(63). For the first coordinate, we identify the region $\Omega_{s}^{\prime}$ bounded by $\operatorname{Re}(z) \in\{-1,1\}$ and $\phi_{s}(L)$ with a rectangle $[-T(s), T(s)] \times[0,1]$ of length $2 T(s)$ by a biholomorphism $\Theta_{s}$. Write $\Theta_{s} \circ u_{s, 1}$ in Fourier coefficients on the neck region $[-T(s), T(s)] \times[0,1]$

$$
\begin{aligned}
\Theta_{s} \circ u_{s, 1}(\tau+i t)=\left(\tau-\tau_{0}(s)\right)+i t & +\sum_{n>0} c_{n}(s) \exp (2 \pi n(\tau-T(s))+i t) \\
& +\sum_{n<0} c_{n}(s) \exp (2 \pi n(\tau+T(s))+i t) .
\end{aligned}
$$

Let $c_{n}(0)$ be the similar Fourier coefficients of the derivative of $\Theta_{s} \circ w_{s, 1}$, where $w_{s, 1}$ is the approximate solution, and $\tau_{0}(0)$ the translation factor of the leading order term of $\Theta_{s} \circ w_{s, 1}$. Convergence of $u_{s, 1}$ to $u_{0,1}$ on compact subsets and agreement of $\Theta_{s} \circ u_{0,1}$ with $\Theta_{s} \circ w_{0,1}$ on a neighborhood of $\tau= \pm T(s)$ (where both are defined) implies that $c_{n}(s) \rightarrow c_{n}(0)$ for all $n$. After a conformal variation that eliminates the difference $\tau_{0}(s)-\tau_{0}(0)$ (that is, a small variation in length of the neck) the difference satisfies

$\left|\Theta_{s} \circ u_{s, 1}(\tau+i t)-\Theta_{s} \circ w_{0,1}(\tau+i t)\right|<C(\exp (-2 \pi(T(s)-\tau))+\exp (-2 \pi(\tau-T(s))))$.

and similarly for the first derivatives. We break $\Omega_{s}^{\prime}$ into three regions separated by the curves $\operatorname{Re}\left(u_{0,1}\right)=s$ : The region $\Omega_{s}$ with $s=1 / \varkappa$ and the regions $\Omega_{s}^{ \pm}$between $\operatorname{Re}(z)\left(u_{0,1}(s)\right) \in\{-s, s\}$ and $\operatorname{Re}(z)\left(u_{0,1}(s)\right) \in\{-1,1\}$. Since $u_{0,1}(z) \sim 1 / z$, the conformal modulus of $\Theta_{s}$ is order $1 / z$ on $\Omega_{s}^{ \pm}$while $\Theta_{s}$ has conformal modulus bounded by $s$ on $\Omega_{s}$. It follows that for any $\epsilon>0$, there exists $s_{0}>0$ such that for $s<s_{0}$

$$
\begin{aligned}
& \int_{[-T(s), T(s)] \times[0,1]}\left(\left|u_{s, 1}(\tau+i t)-w_{s, 1}(\tau+i t)\right|^{p}\right. \\
&\left.+\left|\mathrm{d} u_{s, 1}(\tau+i t)-\mathrm{d} w_{s, 1}(\tau+i t)\right|^{p}\right) e_{\varkappa}(\tau+i t) \mathrm{d} t \mathrm{~d} \tau \\
&
\end{aligned}
$$

where the first term $C_{1} \epsilon$ arises from the contribution of the complement of $\Omega_{s}^{\prime}$ where $u_{s}$ converges uniformly in all derivatives, the second $C_{2} \epsilon$ from the integrals over $\Omega_{s}^{ \pm}$, which have exponentially decaying integrand, and the third $C_{3} \epsilon /(a-\lambda)$ from the integral over $\Omega_{\varkappa}^{\prime}$, on which the integrand is also exponentially decaying at a rate depending on the minimum non-zero angle $a$ between the branches of the Lagrangian. Combining the results for the various components implies that for $t$ sufficiently large, $u_{t}$ is arbitrarily close to the approximate solution $w_{t}$ in the Sobolev norm defined by (63). This proves part (c) of Theorem 8.1 up to sign.

The statement on regularity in Theorem 8.1 follows from Lemmas 8.5 (b) and 8.6 (b). Indeed, for $s$ sufficiently small, the linearized operators are surjectivity so all rigid holomorphic disks with boundary on $\phi_{s}$ are regular. 
Finally we show that the gluing map in Theorem 8.1 (c) is orientation preserving. The sign computation for the gluing map is similar to that for the $A_{\infty}$ associativity relation (48), but in this case the determinant lines associated to the nodes $q_{k} \in S$ mapping to the self-tangency $v \in X$ have index shifted by one, which creates additional signs. For the purpose of computing orientations it suffices to consider the case that there is a single node $q_{k}$ mapping to a tangency. Recall that for any self-intersection point $x_{j} \in \mathcal{I}^{\text {si }}\left(\phi_{t}\right)$ the notation $\mathbb{D}_{x_{j}, 2}^{ \pm}$denotes the determinant line associated to $x_{j}$ in the Fredholm operator on the once-punctured disk associated to a choice of path from $\gamma_{x}$. By assumption in (30), the orientations on $\mathbb{D}_{x_{j}, 2}^{ \pm}$are defined so that the tensor products $\mathbb{D}_{x_{j}, 2}^{-} \otimes \mathbb{D}_{x_{j}, 2}^{+} \cong \mathbb{R}$ with the standard orientation on the trivial vector space, in the case of a self-transverse boundary condition, or a one-dimensional vector space, in the case of self-tangent boundary condition $x_{j}=v$; to simplify notation we assume that each vertex maps to a self-intersection point; otherwise the following discussion holds by the same argument replacing $\mathbb{D}_{x_{j}, 2}$ with $\mathbb{D}_{x_{j}, 1}$ in the notation for each vertex mapping to a Morse unstable manifold. By deforming the parametrized linear operator $\tilde{D}_{u}$ of (25) to the linearized operator $D_{u}$ plus a trivial operator, one obtains an isomorphism of determinant lines

$$
\operatorname{det}\left(T \mathcal{M}_{d}\left(x_{0}, \ldots, x_{d}\right)\right) \rightarrow \operatorname{det}\left(T \mathcal{M}_{d}\right) \mathbb{D}_{x_{0}, 2}^{+} \mathbb{D}_{x_{1}, 2}^{-} \ldots \mathbb{D}_{x_{d}, 2}^{-} .
$$

The gluing map for a single node takes the form (omitting tensor products from the notation to save space)

$$
\operatorname{det}\left(T \mathcal{M}_{m}\right) \mathbb{D}_{v, 2}^{+} \mathbb{D}_{x_{n+1}, 2}^{-} \ldots \mathbb{D}_{x_{n+m}, 2}^{-} \operatorname{det}\left(T \mathcal{M}_{d-m+1,2}\right) \mathbb{D}_{x_{0}, 2}^{+} \mathbb{D}_{x_{1}, 2}^{-} \ldots \mathbb{D}_{v, 2}^{-} \ldots \mathbb{D}_{x_{d}, 2}^{-}
$$

To determine the sign of this map, first note that the gluing map

$$
(0, \epsilon) \times \mathcal{M}_{m} \times \mathcal{M}_{d-m+1} \rightarrow \mathcal{M}_{d}
$$

on the associahedra $\mathcal{M}_{k}$ is given in coordinates (using the automorphisms to fix the location of the first and second point in $\mathcal{M}_{m}$ to equal 0 resp. 1 and $\mathcal{M}_{d-m+1}$ ) by

$$
\begin{aligned}
& \left(\delta,\left(z_{3}, \ldots, z_{m}\right),\left(w_{3}, \ldots, w_{d-m+1}\right)\right) \\
& \rightarrow\left(w_{3}, \ldots, w_{n+1}, w_{n+1}+\delta, w_{n+1}+\delta z_{3}, \ldots, w_{n+1}+\delta z_{m}, w_{n+2}, \ldots, w_{d-m}\right) .
\end{aligned}
$$

The map (68) acts on orientations by a sign of -1 to the power

$$
(m-1)(n-1) \text {. }
$$

These signs combine with the contributions

$$
\sum_{k=1}^{n} k\left|x_{k}\right|+(n+1)(|v|+1)+\sum_{k=n+m+1}^{d}(k-m+1)\left|x_{k}\right|+\sum_{k=n+1}^{m}(k-n)\left|x_{k}\right|
$$


in the definition of the structure maps, and a contribution

$$
(d-m+1) m+m\left(|v|+\sum_{i \geq n}\left|x_{i}\right|\right)
$$

from permuting the determinant lines $\mathbb{D}_{x_{j}, 2}^{-}, j=n+1, \ldots, n+m, \mathbb{D}_{v, 2}^{+}$with $\operatorname{det}\left(T \mathcal{M}_{d-m+1}\right)$ and permuting these determinant lines with the $\mathbb{D}_{x_{i}, 2}^{-}, i \leq n, \mathbb{D}_{v, 2}^{-}$. The extra factor of $\mathbb{R}$ created by the gluing at the self-tangency point can be moved to the first position at the cost of creating signs in the number of

$$
\sum_{k=0}^{n}\left|x_{k}\right|+d-1 .
$$

Combining the signs (69), (70), (71), (72) one obtains mod 2

$$
\begin{gathered}
(m n+n+m)+\left(\sum_{k=1}^{n} k\left|x_{k}\right|+(n+1)(|v|+1)+\sum_{k=n+m+1}^{d}(k-m+1)\left|x_{k}\right|+\sum_{k=n+1}^{n+m}(k-n)\left|x_{k}\right|\right) \\
\quad+(d-m+1) m+m\left(|v|+\sum_{i \leq n}\left|x_{i}\right|\right)+\sum_{k=1}^{n}\left|x_{k}\right|+d+1 \\
\equiv(m n+m+n)+\sum_{k=1}^{d} k\left|x_{k}\right|+(n+1)(|v|+1)+\sum_{k=n+m+2}^{d}(m-1)\left|x_{k}\right|+\sum_{k=n+1}^{n+m} n\left|x_{k}\right| \\
\quad+(d-m+1) m+m\left(d+\sum_{i \geq n+m+1}\left|x_{i}\right|\right)+\sum_{k=0}^{n}\left|x_{k}\right|+d+1
\end{gathered}
$$

$$
\begin{gathered}
\equiv m n+m+\sum_{k=1}^{d} k\left|x_{k}\right|+|v|+\sum_{k=n+m+2}^{d}\left|x_{k}\right|+n m+(d-m+1) m+m d+\sum_{k=0}^{n}\left|x_{k}\right| \\
\equiv m+\sum_{k=1}^{d} k\left|x_{k}\right|+\sum_{k=n+1}^{n+m}\left|x_{k}\right|+m+\sum_{k=n+m+2}^{d}\left|x_{k}\right|+\sum_{k=0}^{n}\left|x_{k}\right| .
\end{gathered}
$$

After incorporating the sign (47) the number of signs is congruent mod 2 to

$$
\sum_{k=0}^{d}\left|x_{k}\right|+d \equiv 0
$$

This completes the proof of Theorem 8.1. 

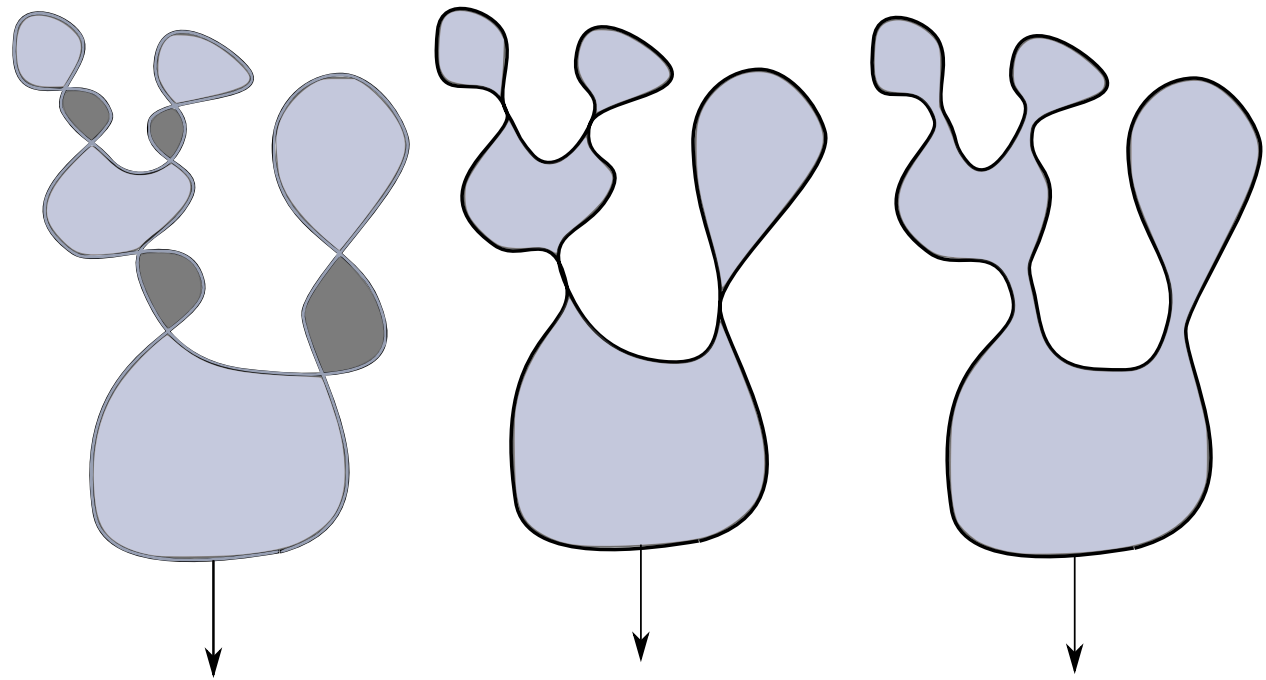

Figure 19: Disks on an immersion developing self-tangent boundary

Corollary 8.7 Let $\phi_{s}: L \rightarrow X$ be an admissible Maslov flow with a self-tangency at $s=0$ as above. For any $E>0$ and $\underline{x}=\left(x_{0}, \ldots, x_{d}\right)$ there exists an $\epsilon_{0}$ so that for $\epsilon<\epsilon_{0}$ there is an orientation-preserving bijection

$$
\begin{aligned}
& \left\{u \in \mathcal{M}_{d}\left(\phi_{-\epsilon}, \underline{x}\right)_{\rho} \mid E(u)<E\right\} \\
& \quad \rightarrow\left\{\underline{u} \in \mathcal{M}\left(\phi_{\epsilon}, \underline{x}_{0}\right)_{\rho} \times_{\mathcal{I}\left(\phi_{\epsilon}\right)^{r}} \prod_{i=1}^{r} \mathcal{M}\left(\phi_{\epsilon}, v_{\epsilon_{i}}, \underline{x}_{i}\right)_{\rho} \mid E(\underline{u})<E\right\}
\end{aligned}
$$

where $\underline{x}_{0}=\left(x_{0}, v_{-\kappa_{i}}\right)_{i=1}^{r}$ with $\kappa_{i} \in\{ \pm 1\}$ and $\underline{x}=\underline{x}_{1} \cup \ldots \cup \underline{x}_{r}$, where $r$ runs over non-negative integers; see Figure 19.

Proof We combine the different parts of Theorem 8.1: Given a family $u_{\epsilon} \in \mathcal{M}^{<E}\left(\phi_{\epsilon}\right)$, we obtain in the limit $\epsilon \rightarrow 0$ an element $u \in \mathcal{M}^{<E}\left(\phi_{0}\right)$. Removing the preimages $u^{-1}\left(x_{j}\right)$ of the tangency points in $\phi_{0}(L)$ we obtain a disconnected domain $C-\left\{u^{-1}\left(x_{j}\right)\right\}$. Define $u_{0}$ to be the closure of the component of $C-\left\{u^{-1}\left(x_{j}\right)\right\}$ containing the outgoing edge $e_{0}$, and let $u_{1}, \ldots, u_{r}$ be the components of $u$ attached to the component $u_{0}$ at the self-tangency points. By Theorem 8.1 again, each of these shrinks to a component mapping to $v_{ \pm \kappa_{i}}$ for $\epsilon<0$, and these are connected by small strips connecting $v_{ \pm \kappa_{i}}$ with $v_{\mp \kappa_{i}}$. The bijection (76) follows. 


\section{Invariance for birth-death singularities}

In this section we use the correspondence between holomorphic curves with Lagrangian boundary condition evolving under Maslov flow in Theorem 8.1 to construct the claimed map in Theorem 1.1 between Maurer-Cartan moduli spaces as the immersion develops (or loses) a pair of self-intersection points.

We first introduce some notation for counting curves in the immersion with selftangency. Let $\overline{\mathcal{M}}\left(\phi_{0}\right)$ denote the moduli space of treed holomorphic disks with boundary condition on $\phi_{0}$ and let $\overline{\mathcal{M}}\left(\phi_{0}\right)_{0}$ denote the part of the moduli space of expected dimension zero with the property that any combinatorially-finite edge $T_{e} \subset$ $T, e \in \operatorname{Edge}_{<\infty}(\Gamma)$ mapping to the self-tangency $v$ has length zero:

$$
\left(u\left(T_{e}\right)=v\right) \Longrightarrow(\ell(e)=0) .
$$

(Without this condition, treed disks splitting at the tangency cannot be rigid, since one could add an edge of arbitrary length.) Theorem 8.1 implies that for any energy bound $E$, the subset of rigid holomorphic treed disks $\overline{\mathcal{M}}^{E}\left(\phi_{0}\right)_{\rho}$ of energy at most $E$ is compact, since the correspondence in Theorem 8.1 is energy preserving up to a small constant determined by the change in action at the self-intersection points. Each element $u: C \rightarrow X$ of $\overline{\mathcal{M}}\left(\phi_{0}\right)_{\rho}$ has underlying combinatorial type a treed nodal disk $C$ where the nodes of $C$ map to the self-tangency point $v \in X$. For any such map let $E(u)$ denote the energy, $\sigma(u)$ the number of interior markings, and $y(u)$ the monodromy of the local system.

Definition 9.1 (Composition maps for self-tangent immersions) Define maps similar to the $A_{\infty}$ structure maps

$$
\begin{aligned}
\mu_{d}\left(\phi_{0}\right): & C F\left(\phi_{0}\right)^{d} \rightarrow C F\left(\phi_{0}\right), \\
\mu_{d}\left(\phi_{0} ; x_{1}, \ldots, x_{d}\right):= & \sum_{u \in \overline{\mathcal{M}}\left(\phi_{0} ; x_{0}, \ldots, x_{d}\right) \rho}(-1)^{\ominus}(\sigma(u) !)^{-1} y(u) q^{E(u)} \epsilon(u) x_{0}
\end{aligned}
$$

where $\varnothing$ is as in (47).

Remark 9.2 The maps (77) fail to satisfy the $A_{\infty}$ axiom. Suppose that the orientations on the determinant lines at the self-tangency points $\operatorname{det}\left(D_{x_{\varkappa}}^{ \pm}\right)$are chosen so that the natural maps $\operatorname{det}\left(D_{x_{0}}^{ \pm}\right) \rightarrow \operatorname{det}\left(D_{x_{\varkappa}}^{ \pm}\right)$induced by the deformation of boundary condition is orientation preserving. If we preclude the tangencies as inputs or outputs then the maps $\mu_{d}\left(\phi_{0}\right)$ capture the limit of the Fukaya composition maps for $\phi_{t}$ as $t \rightarrow 0^{-}$: Let

$$
\Pi: C F\left(\phi_{0}\right) \rightarrow C F\left(\phi_{0}\right), \quad x \mapsto \begin{cases}x & x \in\{v, \bar{v}\} \\ 0 & \text { otherwise }\end{cases}
$$


denote projection onto $\operatorname{span}\{v, \bar{v}\}$ so that $(1-\Pi)$ is the projection of $C F\left(\phi_{0}\right)$ onto the image of $C F\left(\phi_{t}\right)$. Under the natural inclusion $C F\left(\phi_{t}\right) \rightarrow C F\left(\phi_{0}\right)$ we have

$$
\lim _{t \rightarrow 0^{-}} \mu_{d}\left(\phi_{t}\right)=(1-\Pi) \mu_{d}\left(\phi_{0}\right)(1-\Pi)^{d} .
$$

In particular, the maps $(1-\Pi) \mu_{d}\left(\phi_{0}\right)(1-\Pi)^{d}$ satisfy the $A_{\infty}$ associativity axiom, by Theorem 8.1 (b).

However, because of the failure of gluing at a self-tangency without moving the Lagrangian, one cannot expect the maps $\mu_{d}\left(\phi_{0}\right)$ to satisfy the $A_{\infty}$ axiom if some of the semi-infinite edges map to the self-tangent point $v$. For $b \in C F\left(\phi_{0}\right)$ define $b$-deformed maps

$$
\begin{aligned}
& \mu_{d}^{b}\left(\phi_{0} ; a_{1}, \ldots, a_{d}\right) \\
& =\sum_{i_{1}, \ldots, i_{d+1}} \mu_{d+i_{1}+\ldots+i_{d+1}}(\phi_{0}, \underbrace{b, \ldots, b}_{i_{1}}, a_{1}, \underbrace{b, \ldots, b}_{i_{2}}, a_{2}, b, \ldots, b, a_{d}, \underbrace{b, \ldots, b}_{i_{d+1}}) .
\end{aligned}
$$

Definition 9.3 (Curve counts at the tangencies) Define two new maps composing resp. precomposing with projection onto the span of the self-tangency points: Define

$$
\nu_{d, \pm}^{b}: C F\left(\phi_{0}\right)^{\otimes d} \rightarrow C F\left(\phi_{0}\right), \quad \nu_{d,+}^{b}=\Pi \circ \mu_{d}^{b}, \quad \nu_{d,-}^{b}=\mu_{d}^{b} \circ \Pi^{d} .
$$

The maps $\nu_{d, \pm}^{b}$ count holomorphic tree disks with boundary insertion such that the only allowed outputs resp. inputs are the self-tangency points. Define

$$
\iota: C F\left(\phi_{0}\right) \rightarrow C F\left(\phi_{t}\right), \quad v_{0, \pm} \mapsto \epsilon_{ \pm} \bar{v}_{t, \mp}
$$

where $\epsilon_{ \pm} \in\{ \pm 1\}$ are the signs of the small strips in Theorem 8.1. Let $A_{t}$ denote the area of the small strip in Theorem 8.1. Since

$$
\lim _{t \rightarrow 0} A_{t}=0, \quad \lim _{t \rightarrow 0} \operatorname{val}_{q}\left(\nu_{0, \pm}^{b}(1)\right)>0
$$

we have positive $q$-valuation

$$
\operatorname{val}_{q}\left(q^{-A_{t}} \iota\left(\nu_{0,+}^{b}(1)\right)\right)>0 .
$$

We assume that the orientations for the determinant lines at the self-intersection points are chosen so that the contribution of the small strip connecting the self-intersection points is negative.

Definition 9.4 The correction map between Floer cochain spaces is the map

$$
\psi: C F\left(\phi_{-\epsilon}\right) \rightarrow C F\left(\phi_{\epsilon}\right), \quad b_{-\epsilon} \mapsto b_{\epsilon}:=b_{-\epsilon}+q^{-A(\epsilon)} \iota\left(\nu_{0,+}^{b-\epsilon}(1)\right) .
$$




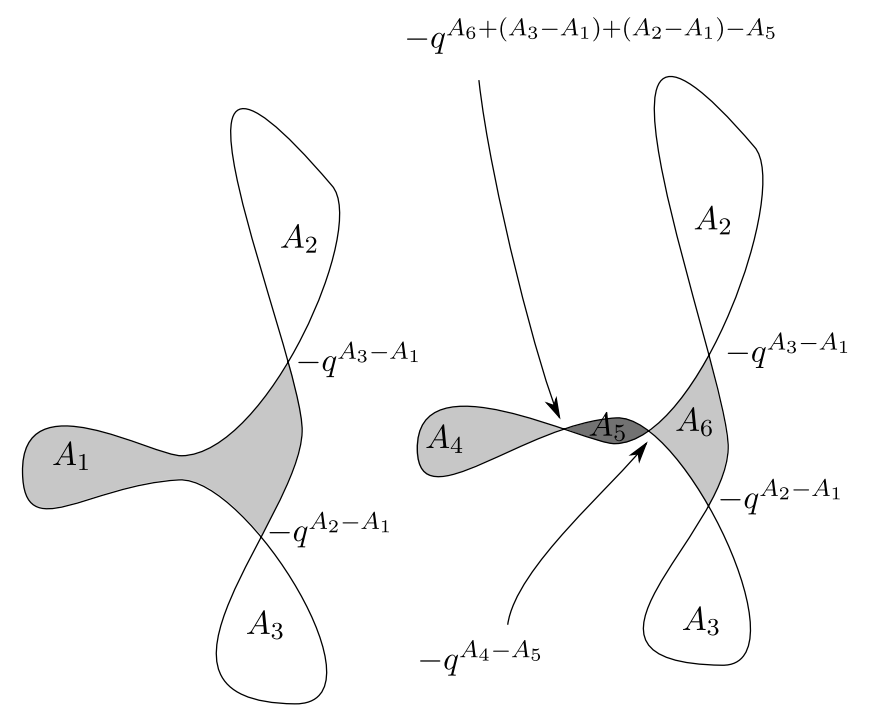

Figure 20: Two weakly bounding cochains related by $\psi$

The informal version of this definition was described in the introduction in (2). The derivative of the map (80) is the identity plus corrections from holomorphic tree disks with output on the tangency and inputs the Maurer-Cartan element, except for a single input with the given tangent vector $x \in \mathcal{I}\left(\phi_{-\epsilon}\right)$ :

$$
D_{b_{-\epsilon}} \psi(x)=x+\iota \circ \nu_{0,+}^{b_{-\epsilon}}(x) .
$$

We show below that (81) gives an isomorphism of Floer cohomologies.

Example 9.5 Two weakly bounding cochains $b_{-\epsilon}$ (left) and $b_{\epsilon}$ (right) related by $\psi$ are shown in Figure 20, with areas shown in the limit $\epsilon \rightarrow 0$ (that is, without any Maslov flow). In this case the area of the small strip $A(\epsilon)$ between the new self-intersection points is equal to the area labelled $A_{5}$. The original weakly bounding cochain $b_{-\epsilon}$ is supported at the two self-intersection points $\bar{v}_{\epsilon, \pm}$ in the boundary of the region with area $A_{1}$, and so the correction in this case is

$$
\iota\left(\nu_{0,+}^{b_{-\epsilon}}(1)\right)=q^{A_{6}+\left(A_{3}-A_{1}\right)+\left(A_{2}-A_{1}\right)} \bar{v}_{\epsilon,+}+q^{A_{4}} \bar{v}_{\epsilon,-} .
$$

This ends the example.

In preparation for the proof of Theorem 1.1, we suppose without loss of generality that $\phi_{t}$ undergoes a birth of two new self-intersection points at $t=0$; the case of a death is similar. 
Lemma 9.6 The equality holds

$$
\lim _{\epsilon \rightarrow 0} \mu_{0}^{b_{-\epsilon}}\left(\phi_{-\epsilon} ; 1\right)=\lim _{\epsilon \rightarrow 0} \mu_{0}^{b_{\epsilon}}\left(\phi_{\epsilon} ; 1\right)
$$

using the inclusion $C F\left(\phi_{-\epsilon}\right) \rightarrow C F\left(\phi_{\epsilon}\right)$.

Proof By definition

$$
\begin{aligned}
\mu_{0}^{b_{\epsilon}}\left(\phi_{\epsilon} ; 1\right) & =\sum_{d \geq 0} \mu_{d}\left(\phi_{\epsilon} ; b_{\epsilon}, \ldots, b_{\epsilon}\right) \\
& =\sum_{d \geq 0} \mu_{d}\left(\phi_{\epsilon} ;\left(b_{\epsilon}-b_{-\epsilon}\right)+b_{-\epsilon}, \ldots,\left(b_{\epsilon}-b_{-\epsilon}\right)+b_{-\epsilon}\right) \\
& =\sum_{r \geq 0} \mu_{r}^{b_{-\epsilon}}\left(\phi_{\epsilon} ; b_{\epsilon}-b_{-\epsilon}, \ldots, b_{\epsilon}-b_{-\epsilon}\right) .
\end{aligned}
$$

By Definition 9.4, this is equal to

$$
\mu_{0}^{b_{\epsilon}}\left(\phi_{\epsilon} ; 1\right)=\sum_{r \geq 0} \mu_{r}^{b_{-\epsilon}}\left(\phi_{\epsilon} ; \iota \nu_{0,+}^{b_{-\epsilon}}(1), \ldots, \iota \nu_{0,+}^{b_{-\epsilon}}(1)\right) .
$$

Step 1: The coefficient of any generators not equal to $v_{ \pm \epsilon}, \bar{v}_{ \pm, \epsilon}$ in (82) are equal. Corollary 8.7 implies that, accounting for both curves that degenerate to curves passing through the self-tangency as well as the change in bounding cochain, the coefficient of any $x \in \mathcal{I}\left(\phi_{\epsilon}\right)-\left\{v_{ \pm, \epsilon}, \bar{v}_{ \pm, \epsilon}\right\}$ are equal. Indeed, any configuration contributing to $\mu_{0}^{b-\epsilon}(1)$ transforms into a collection of holomorphic treed disks with output at $v_{ \pm, \epsilon}$, a collection of small strips connecting to $\bar{v}_{ \pm, \epsilon}$, and holomorphic treed disks with the original output. The difference in areas and holonomies are accounted for by the definition of (80). An example of a configuration counted for $\epsilon>0$ and the matching configuration for $\epsilon<0$ is given in Figure 19 where the contribution for $\epsilon>0$ is of the form $\mu_{2}\left(\nu_{0,+}, \nu_{0,+}\right)$; disks with boundary in the given immersion are lightly shaded while the contributions from $\nu_{0,+}$ are darkly shaded. The signs agree by Theorem 8.1.

Step 2: The coefficient of $v_{ \pm, \epsilon}$ in (82) vanishes on both sides. Recall that the generators $v_{ \pm, \epsilon}$ are the outputs of the small strip in Theorem 8.1. If there are configurations contributing to $\mu_{0}^{b_{\epsilon}}\left(\phi_{\epsilon} ; 1\right)$ with output $v_{ \pm, \epsilon}$ with positive coefficient, then the definition of $\psi$ in (80) implies that these configurations are cancelled by $\mu_{1}\left(q^{-A(\epsilon)} \iota\left(\nu_{0,+}^{b_{-\epsilon}}(1)\right)\right.$. Furthermore, there are no other composition maps $\mu_{r}, r>1$ containing $\iota\left(\nu_{0,+}^{b_{-\epsilon}}(1)\right)$ as an input, since any polygon projects to a polygon in the first coordinate and so the only polygon with input $\bar{v}_{ \pm, \epsilon}$ is the small strip. 


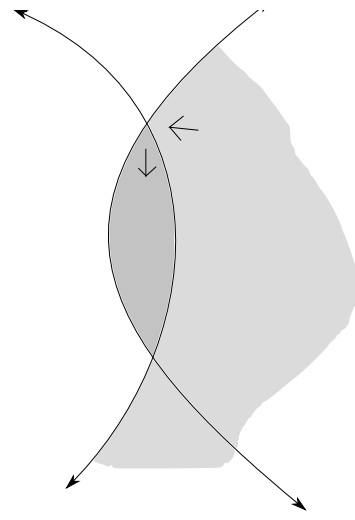

Figure 21: Part of a disk with output at $\bar{v}_{+, \epsilon}$

Step 3: The coefficient of $\bar{v}_{ \pm, \epsilon}$ in (82) vanishes on both sides. Suppose otherwise, so that without loss of generality the coefficient of $\bar{v}_{+, \epsilon}$ is equal to $c \in \Lambda$. The Bianchi $A_{\infty}$ relation for $\phi_{\epsilon}$ reads

$$
\mu_{1}^{b_{\epsilon}} \mu_{0}^{b_{\epsilon}}(1)=0 .
$$

Contributions to this identity include once-broken treed disks with no inputs and output at $v_{-, \epsilon}$, as in the larger shaded region in Figure 21 only a part of whose boundary is shown.

The contribution from configurations with breaking at $\bar{v}_{+, \epsilon}$ is equal to $c q^{A(\epsilon)}$, since the only holomorphic treed disk from $\bar{v}_{+, \epsilon}$ to $v_{-, \epsilon}$ is the small strip discussed above, shown darkly shaded in Figure 21. To see this, note that the projection on the first coordinate is holomorphic and the only holomorphic polygon in the two-dimensional picture is the bigon in Theorem 8.1, shown in Figure 21. Contributions also include configurations with breaking at some other generator $x \in \mathcal{I}\left(\phi_{\epsilon}\right)$, not equal to $v_{ \pm, \epsilon}, \bar{v}_{ \pm, \epsilon}$ and so corresponding to a generator of $\mathcal{I}\left(\phi_{-\epsilon}\right)$. However, by assumption, $b_{-\epsilon}$ is a solution the projective Maurer-Cartan equation, and so the coefficient of $x$ in $\mu_{0}^{b-\epsilon}\left(\phi_{-\epsilon} ; 1\right)$ vanishes. But then by Step 1, the coefficient of $x$ in vanishes as well. Hence the coefficient of $\bar{v}_{+, \epsilon}$ vanishes.

Proof of Theorem 1.1 By Lemma 9.6

$$
\begin{aligned}
b_{-\epsilon} \in M C\left(\phi_{-\epsilon}\right) & \Longrightarrow \mu_{0}^{b_{-\epsilon}}(1) \in \operatorname{span}\left(1_{\phi_{-\epsilon}}\right) \\
& \Longrightarrow \mu_{0}^{b_{\epsilon}}(1) \in \operatorname{span}\left(1_{\phi_{\epsilon}}\right) \\
& \Longrightarrow b_{\epsilon} \in M C\left(\phi_{\epsilon}\right)
\end{aligned}
$$


with the same value of the potential.

To prove the claim on Floer cohomology first note that the Floer differential $\mu_{1}^{b_{ \pm \epsilon}}$ is the derivative of $\mu_{0}^{b_{ \pm \epsilon}}$, which implies by Lemma 9.6 that $D \psi$ is a chain map, where $\psi$ is as in Definition 9.4. Indeed we have

$$
\begin{aligned}
\lim _{\epsilon \rightarrow 0} D \psi\left(\mu_{1}^{b_{-\epsilon}}\right)(x) & =\lim _{\epsilon \rightarrow 0} \frac{d}{d t} \psi\left(\mu_{0}^{b_{-\epsilon}+t x}(1)\right) \\
& =\lim _{\epsilon \rightarrow 0} \frac{d}{d t} \mu_{0}^{\left(b_{-\epsilon}\right)+t D \psi_{b_{-\epsilon}}(x)}(1) \\
& =\lim _{\epsilon \rightarrow 0} \mu_{1}^{\psi\left(b_{-\epsilon}\right)}\left(D \psi_{b_{-\epsilon}}(x)\right) .
\end{aligned}
$$

To show that $D \psi$ induces an isomorphism, we apply the spectral sequence for the cohomology induced by the filtration by powers of $q^{\delta}$, for $\delta$ larger than $\epsilon$ but smaller than the area of any other non-constant holomorphic treed disk. The differential for the first page of the spectral sequence is the Morse differential, together with the map $\bar{v}_{\epsilon, \pm} \rightarrow q^{A(\epsilon)} v_{\epsilon, \mp}$ induced by the small strip, and any cohomology class for this differential has a representative supported in the image of $C F\left(\phi_{-\epsilon}\right)$ in $C F\left(\phi_{\epsilon}\right)$. In particular, $D \psi$ is an isomorphism on the first page, and it follows that $D \psi$ is an isomorphism $H F\left(\phi_{-\epsilon}\right) \rightarrow H F\left(\phi_{\epsilon}\right)$ on the limits of the spectral sequence (see [60, 5.2.12].)

Conversely we claim that the limit of any family of Maurer-Cartan solutions is obtained by the correction formula in (80). Given $b_{\epsilon} \in M C\left(\phi_{\epsilon}\right)$ write

$$
b_{\epsilon}=b_{-\epsilon}+\delta b, \quad \delta b=\sum_{x \in\left\{v_{+}, \bar{v}_{+}, \bar{v}_{-}, v_{-}\right\}}\langle\delta b, x\rangle x .
$$

Consider the equations

$$
\lim _{\epsilon \rightarrow 0} \mu_{0}^{b_{\epsilon}}(1)=\lim _{\epsilon \rightarrow 0} \sum \mu_{d}^{b_{-\epsilon}}(\delta b, \ldots, \delta b) \in \Lambda 1_{\phi_{1}} .
$$

Re-write this equation as

$$
\begin{aligned}
& \left.-\mu_{1}\left(\left\langle\delta b, \bar{v}_{-}\right\rangle \bar{v}_{-}+\left\langle\delta b, \bar{v}_{+}\right\rangle \bar{v}_{+}\right\rangle\right) \\
& \left.\quad=\sum_{d \geq 0} \mu_{d}^{b_{-\epsilon}}(\delta b, \ldots, \delta b)-\mu_{1}\left(\left\langle\delta b, \bar{v}_{-}\right\rangle \bar{v}_{-}+\left\langle\delta b, \bar{v}_{+}\right\rangle \bar{v}_{+}\right\rangle\right) \bmod \operatorname{span}\left(1_{\phi}\right)
\end{aligned}
$$

The terms in $\delta b \bmod q^{n \epsilon}$ determine the terms in $\delta b \bmod q^{n \epsilon+\hbar}$ for some energy quantization constant $\hbar$ and any integer $n$. Therefore this equation has a unique solution $\delta b$, necessarily equal to the one given above in (80). 
Remark 9.7 In Etnyre-Ekholm-Sullivan [25] the authors give a second proof of invariance of Legendrian contact homology under a self-tangency move by using a "bifurcation analysis". It would be interesting to know whether a stabilization or cobordism argument would remove the need for explicit gluing results used above.

Proof of Theorem 1.3 Theorem 1.3 follows from Theorems 1.2 and 1.1 by breaking up the domain of the isotopy $[0, T]$ into sub-intervals $\left[0, t_{1}\right],\left[t_{1}, t_{2}\right], \ldots,\left[t_{k-1}, T\right]$. By induction, we may assume that the theorem holds for any Maslov flow $\phi_{t}$ on the interval $t \in\left[t_{1}, T\right]$. Suppose that $\left[b_{0}\right] \in \overline{M C}\left(\phi_{0}\right)$ with

$$
\min _{i=0}^{k} A_{i}-(\operatorname{dim}(L)-1)\left(T-t_{i}\right)>0 \text {. }
$$

Since $A_{0}=\operatorname{val}_{q}\left(b_{0}\right)$, Theorem 1.2 implies that there exists a family $b_{t}=E_{0}^{t}\left(b_{0}\right), t \in$ $\left[0, t_{1}\right]$ and an identification $H F\left(\phi_{0}, b_{0}\right) \cong H F\left(\phi_{t}, b_{t}\right)$ for all $t$. Theorem 1.1 implies that the family $b_{t}$ continues as a family $b_{t,+} \in M C\left(\phi_{t}\right)$ for $t \in\left[t_{1}, t_{1}+\epsilon\right]$ for $\epsilon$ small with a $q$-valuation

$$
\begin{aligned}
\operatorname{val}_{q}\left(b_{t_{1},+}\right) & =\operatorname{val}_{q}\left(\psi\left(b_{t_{1},-}\right)\right) \\
& =\min \left(\operatorname{val}_{q}\left(b_{t_{1},-}\right), A_{1}\right) \geq \min \left(\operatorname{val}_{q}\left(b_{0}\right)-t_{1}(\operatorname{dim}(L)-1), A_{1}\right) .
\end{aligned}
$$

Now $b_{t_{1},+}$ satisfies the assumption of Theorem 1.3 for the interval $\left[t_{1}, T\right]$ since, letting $A_{1}^{\prime}=\operatorname{val}_{q}\left(b_{t_{1},+}\right)$, we have

$$
\begin{aligned}
\min & \left(A_{1}^{\prime}-(\operatorname{dim}(L)-1)\left(T-t_{1}\right), \min _{i=2}^{k} A_{i}-(\operatorname{dim}(L)-1)\left(T-t_{i}\right)\right) \\
\geq & \min \left(\min \left(\operatorname{val}_{q}\left(b_{0}\right)-T(\operatorname{dim}(L)-1), A_{1}-(\operatorname{dim}(L)-1)\left(T-t_{1}\right)\right),\right. \\
& \left.\min _{i=2}^{k} A_{i}-(\operatorname{dim}(L)-1)\left(T-t_{i}\right)\right) \\
= & \min _{i=0}^{k} A_{i}-(\operatorname{dim}(L)-1)\left(T-t_{i}\right)>0 .
\end{aligned}
$$

By the inductive hypothesis, there exists $b_{T} \in M C\left(\phi_{T}\right)$ with

$$
\begin{gathered}
W\left(\phi_{T}, b_{T}\right)=q^{2\left(T-t_{1}\right)} W\left(\phi_{t_{1}}, b_{t_{1}}\right) \cong q^{2 T} W\left(\phi_{0}, b_{0}\right) \\
H F\left(\phi_{T}, b_{T}\right) \cong H F\left(\phi_{t_{1}}, b_{t_{1}}\right) \cong H F\left(\phi_{0}, b_{0}\right) .
\end{gathered}
$$

The statement of the Theorem for the forward direction follows. The result for the reverse Maslov flow (mean curvature flow) is similar, using inverse Euler flow.

We end the paper with further open questions: 
Remark 9.8 (a) The first question concerns existence of non-trivial objects in the Fukaya category. We conjecture that any rational compact Kähler manifold $(X, \omega)$ contains a Floer non-trivial Lagrangian $\phi: L \rightarrow X, H F(\phi) \neq\{0\}$, that is, a non-trivial object $[\phi]$ in the immersed Fukaya category $\mathcal{F}(X)$, and the definition of Lagrangian is taken to be sufficiently general. This paper shows that this property is preserved under (at least small) Kähler-Ricci flows $\left(X, \omega_{t}\right)$, while the paper [16] shows that sufficiently nice surgeries in the flow generate non-trivial objects.

(b) The next question concerns the existence of mean curvature flows. Joyce's conjecture [38] predicts that after allowing certain singular Lagrangians and surgeries, mean curvature flow $\phi_{t}: L_{t} \rightarrow X, t \in[0, \infty)$ exists and preserves Floer cohomology $\operatorname{HF}\left(\phi_{t}, b_{t}\right)$ if $b_{0} \in M C\left(\phi_{0}\right)$. Note that this approach requires surgery at times $t_{i} \in(0, T)$ that are not singular times of the mean curvature flow. This conjecture is open even for immersed curves $\phi: L \rightarrow X$ on surfaces $X, \operatorname{dim}(X)=2$. Some analytic results relevant to the surgeries necessary when the $q$-valuation of the weakly bounding cochain become zero are contained in the manuscript [31, Chapter 10].

(c) To what extent is non-displaceability equivalent to Floer non-triviality? In the case of immersions in the two-sphere $\phi: L \cong S^{1} \rightarrow X \cong S^{2}$, non-vanishing of Floer cohomology $H F(\phi, b)$ seems to be stronger than non-displaceability $\exists \psi: X \rightarrow X, \psi(\phi(L)) \cap \phi(L)$ empty. Is there a version of Floer theory (such as bulk deformations) that detects non-displaceability?

(d) The structure of the space of Maurer-Cartan solutions $M C(\phi)$ seems poorly understood, even in the case of immersions $\phi: L \cong S^{1} \rightarrow X \cong S^{2}$ in twosphere. For example, what is the dimension of $M C(\phi)$ ?

(e) Immersed Lagrangian Floer theory is somewhat limited in applicability by the requirement that the transverse self-intersection condition: $\operatorname{HF}(\phi, b)$ is defined only for immersions $\phi: L \rightarrow X$ with self-transverse, or at least clean, selfintersection $L \times_{\phi} L-\Delta_{L}$. Of course, one can perturb $\phi$ to achieve a selftransverse immersion $\tilde{\phi}$. How does the Maurer-Cartan moduli space $\operatorname{MC}(\tilde{\phi})$ depend on the choice of perturbation $\tilde{\phi}$ ? In particular, is there a "good locus" $M C(\tilde{\phi})$ of weakly bounding cochains $\tilde{b}$ so that the Floer cohomology $\operatorname{HF}(\tilde{\phi}, \tilde{b}), \tilde{b} \in M C(\tilde{\phi})$ is independent of the choice of perturbation?

(f) As a special case of the previous question, does there exist a good Floer theory for Lagrangian immersions that are finite covers of their image? Let $\phi: L \rightarrow X$ be a Lagrangian embedding and let $\tilde{\phi}: \tilde{L} \rightarrow X$ be the immersion obtained by composing $\phi$ with a finite cover $\pi: \tilde{L} \rightarrow L$. Is the immersed Lagrangian 
Immersed Floer theory and Maslov flows

Floer theory of a self-transverse perturbation of the composition $\psi \circ \tilde{\phi}: \tilde{L} \rightarrow X$ independent of the choice of perturbation $\psi: X \rightarrow X$ ?

(g) What is the sub-category of immersed Fukaya category $\mathcal{F}(X)$ generated by embedded Lagrangians $\phi: L \rightarrow X$ ? The mapping cone construction in Fukaya-OhOhta-Ono [31, Chapter 10] implies that, at least in some cases, self-intersection points $x \in L \times{ }_{\phi} L$ may be replaced with mapping cones $\operatorname{Cone}(x), x \in \operatorname{Hom}(\phi, \phi)$; see also Mak-Wu [44] and Fang [26].

\section{References}

[1] M. Abouzaid. Framed bordism and Lagrangian embeddings of exotic spheres. Ann. of Math. (2) 175 (2012), no. 1, $71\{185$.

[2] M. Akaho and D. Joyce. Immersed Lagrangian Floer Theory. J. Differential Geom. 86 (2010), no. 3, 381-500.

[3] G. Alston and E. Bao. Exact, graded, immersed Lagrangians and Floer theory. arxiv:1407.3871.

[4] S. B. Angenent and J. J. L. Velázquez. Asymptotic shape of cusp singularities in curve shortening. Duke Math. J. 77 (1995), no. 1, 71-110.

[5] S. B. Angenent. Curve shortening and the topology of closed geodesics on surfaces. Annals of Mathematics, 162 (2005), 1187\{1241.

[6] D. Auroux, D. Gayet, and J.-P. Mohsen. Symplectic hypersurfaces in the complement of an isotropic submanifold. Math. Ann., 321(4):739-754, 2001.

[7] C. Baker. The mean curvature flow of submanifolds of high codimension. arxiv:1104.4409.

[8] P. Biran and O. Cornea. Quantum structures for Lagrangian submanifolds. arxiv:0708.4221.

[9] R. Bott and L. W. Tu. Differential forms in algebraic topology. Graduate Texts in Mathematics, 82. Springer-Verlag, New York-Berlin, 1982.

[10] S. Carapetis. Interactive demonstrative of curve-shortening flow. Retrieved January 2018 at http://a.carapetis.com/csf/.

[11] J. Cerf. La stratification naturelle des espaces de fonctions différentiables réelles et le théorème de la pseudo-isotopie. Inst. Hautes Études Sci. Publ. Math., (39):5-173, 1970.

[12] F. Charest. Source Spaces and Perturbations for Cluster Complexes. arxiv:1212.2923.

[13] F. Charest and C. Woodward. Floer trajectories and stabilizing divisors. Fixed Point Theory. June 2017, Volume 19, Issue 2, pp 1165-1236. 
[14] W. Chen and Y. Ruan. Orbifold Gromov-Witten theory. In Orbifolds in mathematics and physics (Madison, WI, 2001), volume 310 of Contemp. Math., pages 25-85. Amer. Math. Soc., Providence, RI, 2002.

[15] K. Cieliebak and K. Mohnke. Symplectic hypersurfaces and transversality in GromovWitten theory. J. Symplectic Geom., 5(3):281-356, 2007.

[16] F. Charest and C. Woodward. Floer theory and flips. arxiv:1508.01573.

[17] J. Chen and J. M.-S. Ma. Geometry of Lagrangian self-shrinking tori and applications to the Piecewise Lagrangian Mean Curvature Flow arxiv:1604.07563.

[18] K. Cieliebak and E. Goldstein. A note on the mean curvature, Maslov class and symplectic area of Lagrangian immersions. J. Symplectic Geom. 2 (2004), no. 2, 261-266.

[19] T. H. Colding and W. .P. Minicozzi II . Generic mean curvature flow I: generic singularities. Ann. of Math. (2) 175 (2012), no. 2, 755-833.

[20] O. Cornea and F. Lalonde. Cluster homology: An overview of the construction and results. Electron. Res. Announc. Amer. Math. Soc., (12):1-12, 2006.

[21] P. Deligne and D. Freed. Sign manifesto. In Quantum Fields and Strings, A course for mathematicians, 2 vols. Amer. Math. Soc. Providence 1999. (web version) Eds. P. Deligne, P. Etingof, D.S. Freed, L. Jeffrey, D. Kazhdan, J. Morgan, D.R. Morrison, E. Witten.

[22] S. K. Donaldson. Symplectic submanifolds and almost-complex geometry. J. Differential Geom. 44 (1996), no. 4, 666-705.

[23] T. Ekholm, J. Etnyre and M. Sullivan. The contact homology of Legendrian submanifolds in $\mathbb{R}^{2 n+1}$. J. Differential Geom. 71 (2005), no. 2, 177-305.

[24] T. Ekholm, J. Etnyre, M. Sullivan. Legendrian contact homology in $P \times \mathbb{R}$. Trans. Amer. Math. Soc. 359 (2007), no. 7, 3301-3335.

[25] T. Ekholm, J. Etnyre, and M. Sullivan. Orientations in Legendrian contact homology and exact Lagrangian immersions. Internat. J. Math., 16(5):453-532, 2005.

[26] K.-Y. Fang. Geometric constructions of mapping cones in the Fukaya category. Ph.D. Thesis, Berkeley, 2018.

[27] A. Floer. Monopoles on asymptotically flat manifolds. In: Hofer H., Taubes C.H., Weinstein A., Zehnder E. (eds) The Floer Memorial Volume. Progress in Mathematics, vol 133. Birkhäuser Basel.

[28] A. Floer. Morse theory for Lagrangian intersections. J. Differential Geom. 28 (1988), no. $3,513-\{547$.

[29] A. Floer. The unregularized gradient flow of the symplectic action. Comm. Pure Appl. Math. 41 (1988), no. 6, 775-813.

[30] U. Frauenfelder and K. Zehmisch. Gromov compactness for holomorphic discs with totally real boundary conditions. J. Fixed Point Theory Appl. 17 (2015), no. 3, 521-540. 
[31] K. Fukaya, Y.-G. Oh, H. Ohta, and K. Ono. Lagrangian intersection Floer theory: anomaly and obstruction., volume 46 of AMS/IP Studies in Advanced Mathematics. American Mathematical Society, Providence, RI, 2009. Orientation chapter at https://www.math.kyoto-u.ac.jp/ fukaya/bookchap9071113.pdf version 2007. Surgery chapter at https://www.math.kyoto-u.ac.jp/ fukaya/Chapter10071117.pdf.

[32] K. Fukaya, Y.-G. Oh, H. Ohta, and K. Ono. Lagrangian Floer theory on compact toric manifolds. I. Duke Math. J., 151(1):23-174, 2010.

[33] S. Ganatra. Symplectic Cohomology and Duality for the Wrapped Fukaya Category. PhD Thesis, Massachusetts Institute of Technology, 2006.

[34] M. Grayson. The heat equation shrinks embedded plane curves to round points. Jour. of Diff. Geom. 26 (2): 285\{314, 1987.

[35] M.A. Grayson. The shape of a figure-eight under the curve shortening flow. Inventiones math 96 (1) 177-180, 1989.

[36] M. Gromov. Proceedings of the international congress of mathematicians. Nice, 1970. Vol. 2, 221\{225; Gauthier-Villars, Paris, 1971.

[37] D. Joyce. UCLhandout2.pdf. Talk slides retrieved 1/17/2018.

[38] D. Joyce. Conjectures on Bridgeland stability for Fukaya categories of Calabi-Yau manifolds, special Lagrangians, and Lagrangian mean curvature flow. EMS Surv. Math. Sci. 2 (2015), 1-62. arxiv:1401.4949.

[39] D. Joyce, Y.-I. Lee, and M. -P Tsui. Self-similar solutions and translating solitons for Lagrangian mean curvature flow. J. Differential Geom. Volume 84, Number 1 (2010), $127-161$.

[40] Yi-Jen Lee. Reidemeister torsion in Floer-Novikov theory and counting pseudoholomorphic tori. I. J. Symplectic Geom. 3 (2005), no. 2, 221-311. Reidemeister torsion in Floer-Novikov theory and counting pseudo-holomorphic tori. II. J. Symplectic Geom. 3 (2005), no. 3, 385-480.

[41] J. A. Lees. On the classification of Lagrange immersions. Duke Math. J. 43 (1976), no. 2, 217-224.

[42] J. D. Lotay and T. Pacini. Coupled flows, convexity and calibrations: Lagrangian and totally real geometry. arxiv:1404.4227.

[43] D. McDuff and D. Salamon. J-holomorphic curves and symplectic topology, volume 52 of American Mathematical Society Colloquium Publications. American Mathematical Society, Providence, RI, 2004.

[44] C. Y. Mak and W. Wu. Dehn twists exact sequences through Lagrangian cobordism arxiv: 1509.08028.

[45] S. Ma'u, K. Wehrheim, and C.T. Woodward. $A_{\infty}$-functors for Lagrangian correspondences. Selecta Math. 24(3), p. 1913-2002, 2018.

[46] J. Moser. On the Volume Elements on a Manifold. Transactions of the American Mathematical Society. Vol. 120, No. 2 (Nov., 1965), pp. 286-294. 
[47] T. Pacini. Maslov, Chern-Weil and Mean Curvature. Jour. of Geom. and Physics 135 (2019) 129-134 arxiv:1711.07928.

[48] J. Palmer and C. Woodward. Invariance of immersed Floer cohomology under Lagrangian surgery. arxiv:1903.01943.

[49] H.L. Royden. Real Analysis. Macmillan Publishing Company, New York, 1988.

[50] F. Schmäschke. Floer homology of Lagrangians in clean intersection. arxiv: 1606.05327.

[51] J.-C. Sikorav. Some properties of holomorphic curves in almost complex manifolds. Holomorphic curves in symplectic geometry, 165\{189, Progr. Math., 117, Birkhäuser, Basel, 1994.

[52] K. Smoczyk. Lagrangian mean curvature flow. Habilitation Thesis, Leipzig, 2001. http://service.ifam.uni-hannover.de/ smoczyk/publications/preprint07.pdf.

[53] M. Schwarz. Morse homology, vol. 111 of Progress in Math. Birkhäuser Verlag, Basel, 1993.

[54] P. Seidel. Graded Lagrangian submanifolds. Bull. Soc. Math. France, 128(1):103-149, 2000.

[55] P. Seidel. Homological mirror symmetry for the genus two curve. J. Algebraic Geom. 20:727-769, 2011.

[56] M. G. Sullivan. $K$-theoretic invariants for Floer homology. Geom. Funct. Anal. 12 (2002), no. 4, 810-872.

[57] R.P. Thomas and S. T. Yau. Special Lagrangians, stable bundles and mean curvature flow. Comm. Anal. Geom. 10 (2002), no. 5, 1075-1113.

[58] S. Warschawski. On the higher derivatives at the boundary in conformal mapping. Trans. Amer. Math. Soc. 38 (1935), no. 2, 310\{340.

[59] K. Wehrheim and C.T. Woodward. Orientations for pseudoholomorphic quilts. arXiv:1503.07803.

[60] C. Weibel. Homological algebra. Cambridge University Press, 1995.

[61] A. Weinstein. Removing intersections of Lagrangian immersions. Illinois J. Math. 27 (1983), no. 3, 484\{500.

[62] File

https://commons.wikimedia.org/wiki/File:Sphere__-_monochrome_simple.svg. retrieved February 2018.

Mathematics - Altgeld Hall, University of Illinois at Urbana Champaign, 1409 W. Green Street, Urbana, IL 61801, U.S.A.

Mathematics-Hill Center, Rutgers University, 110 Frelinghuysen Road, Piscataway, NJ 088548019, U.S.A.

jpalmer5@illinois.edu, woodwardc@gmail.com 